\title{
Towards Evaluating Veracity of Textual Statements on the Web
}

\author{
Qiang Zhang
}

A dissertation submitted in partial fulfillment

of the requirements for the degree of

Doctor of Philosophy

of

University College London.

Department of Computer Science

University College London

October 8, 2021 
I, Qiang Zhang, confirm that the work presented in this thesis is my own. Where information has been derived from other sources, I confirm that this has been indicated in the work. 



\section{Abstract}

The quality of digital information on the web has been disquieting due to the absence of careful checking. Consequently, a large volume of false textual information is being produced and disseminated with misstatements of facts. The potential negative influence on the public, especially in time-sensitive emergencies, is a growing concern. This concern has motivated this thesis to deal with the problem of veracity evaluation.

In this thesis, we set out to develop machine learning models for veracity evaluation of textual claims based on stance and user engagements. Such evaluation is achieved from three aspects: news stance detection, engaged user replies in social media and the engagement dynamics. First of all, we study stance detection in the context of online news articles where a claim is predicted to be true if it is supported by the evidential articles. We propose to manifest a hierarchical structure among stance classes: the high-level aims at identifying relatedness, while the low-level aims at classifying, those identified as related, into the other three classes, i.e., agree, disagree and discuss. This model disentangles the semantic difference of related/unrelated and the other three stances, and helps address the class imbalance problem. Beyond news articles, user replies on social media platforms also contain stances and can infer claim veracity. Claims and user replies in social media are usually short and can be ambiguous; to deal with semantic ambiguity, we design a deep latent variable model with a latent distribution to allow multimodal semantic distribution. Also, marginalising the latent distribution enables the model to be more robust in relatively smalls-sized datasets. Thirdly, we extend the above content-based models by tracking the dynamics of user engagement in misinforma- 
tion propagation. To capture this dynamics, we formulate user engagements as a dynamic graph, and extract its temporal evolution patterns and geometric features based on an attention modified Temporal Point Process. This allows to forecast the cumulative number of engaged users, and can be useful in assessing the threat level of an individual piece of misinformation.

The ability to evaluate veracity and forecast the scale growth of engagement networks serves to practically assist the minimization of online false information's negative impacts. 


\section{Impact Statement}

False information has caused increasing concerns in both academia and industry as it creates a distorted picture of reality for political or financial gains. To address these concerns, this thesis sets out to identify false information on the web and social media. The impacts of the thesis can summarized as three folds.

In academia, this thesis makes technical contributions to veracity evaluation algorithms and relevant techniques. These include (1) an insightful analysis of semantic distributions of news stance classes and a hierarchical solution to the class imbalance problem, (2) a deep latent variable model to deal with the modelling of engaged user replies and their incorporation to misinformation detection, (3) an attention modified Temporal Point Process and its application to dynamics of user engagement in social media. The above works provide general expertise or taskspecific discovery to the communities of machine learning, data mining, and natural language understanding.

This thesis has broad impact to industry. As misinformation is a common problem faced by technical companies such as Google, Facebook and Twitter, we provide discussion and analysis of how to deploy the proposed algorithms in these companies. The employment can be an intermediate step towards the final goal or a prototype of the commercialized version. Besides, the proposed algorithms can pave way for commercialized tools and can boost business in the form of start-ups.

More importantly, this thesis helps reduce the negative influence of misinformation on the society. The thesis develops tools to identify misinformation and forecast its development to measure the threat level. It is therefore an important contribution to building a secure, falsehood-free online environment. 



\section{Acknowledgements}

It is one of the most important milestones in my life to complete the $\mathrm{PhD}$ thesis. Over almost four years' study in UCL, I have learnt to be an independent researcher with the expertise in artificial intelligence. Looking back to the whole process of pursing the $\mathrm{PhD}$ degree, I feel very grateful to those who have helped and influenced me in various ways.

I am super grateful to my supervisor Prof. Emine Yilmaz. I would like to thank her for offering me the valuable opportunity to study with her in UCL - a world-leading research university. Emine led me to the research of artificial intelligence in weekly meetings and helped me build my research career. She is very open-minded and encouraged me to explore my interest. At the same time, she is also academically and emotionally supportive, and always ready to extend a helping hand whenever I need. Emine is greatly active in both academia and industry; she kindly gave me good chances to hone skills in these two scenes and shaped my career in exploring and leading the future research. These have exerted great influence on the way of my becoming an independent researcher.

I am grateful to Dr. Shi Zhou for being my secondary supervisor. Shi also kindly examined my first and transfer viva. From his background and judgment, Shi asked me to more thoroughly consider research issues, ranging from analytical thinking to practical challenges. I am thankful to Dr. Aldo Lipani for his advice and guidance in our daily interaction and paper writing. I would like to thank Prof. Sebastian Riedel and Prof. Maria Liakata for being the examiners of my final viva. They provided critical comments and valuable suggestions on the whole thesis, which firmly helped improve this thesis to a higher level. 
I very much cherish the shared experience and memory with the UCL family. I spent great time with the web intelligence group: Dr. Shangsong Liang, Dr. Rishabh Mehrotra, Dr. Manisha Verma, Dr. Jarana Manotumruksa, Sahan Bulathwela, Omer Kirnap, Fanghua Ye, Yue Feng and Zhengxiang Shi. I also thank the colleagues and friends at the AI centre and the CS department: Prof. Ingemar Cox, Prof. Jun Wang, Dr. Bin Zou, Dr. Rui Luo, Dr. Yaodong Yang, Dr. Ying Wen, Dr. Haifeng Zhang, Dr. Yali Du, Dr. Xu Chen, Dr. Jie Li, Zheng Tian, Minne Li, Yixin Wu, Zhichen Gong and Yuxiang Wu for their kind help and insightful discussion. Special thanks are given to the administrative staff at UCL, namely Sarah Bentley, Dawn Bailey and Wendy Richards, for their frequent help.

I am thankful to have the wonderful roommates and close friends in the UK. Thanks to Goodenough College for providing me such a great living place in central London where I enjoyed amazing time for more than two years. I feel lucky to have Jiangdong Yu as my roommate during the first year of my life in London. At that time, we managed to survive in an totally unfamiliar environment. Together, we dealt with various daily challenges that we never met in China, such as language understanding, food cooking and house renting. We offered emotional support to each other to overcome homesickness, especially during traditional Chinese festivals.

Most importantly, I appreciate my parents and the whole family. Thanks to my mother and my father for giving me a comfortable home environment where I could focus on study and entertainment without worrying about living difficulties. From their words and behaviour, I gradually learnt to be diligent, tough and reliable. Special thanks are given to my grandfather who taught me to be flexible, fearless, ambitious and conscientious. They are inspirational guides in my whole life. I also want to thank my grandmother, my uncles and aunty, my cousins for their significant roles in building up my happy childhood.

Last but not least, I am very grateful to my wife, Yongjiao Xue, for her deep trust and love. I am really appreciative of her whole-hearted support in all aspects. Especially, I feel great to have our lovely daughter, Lezhi (Michelle) Zhang.

The ending of my studentship marks the starting of my new life. 


\section{Contents}

1 Introduction $\quad 25$

1.1 Background \& Motivation . . . . . . . . . . . . . . . 25

1.2 Research Challenges . . . . . . . . . . . . . . . . 28

1.3 Major Contributions . . . . . . . . . . . . . . . 30

1.3.1 Hierarchical Stance Detection . . . . . . . . . . . . . . 30

1.3.2 User Replies Aided Misinformation Detection . . . . . . . 31

1.3.3 Detection and Forecast via Engagement Dynamics . . . . . . 32

1.4 Thesis Overview . . . . . . . . . . . . . . . . . 33

1.5 Supporting Publications . . . . . . . . . . . . . . . 34

2 Literature Review $\quad 35$

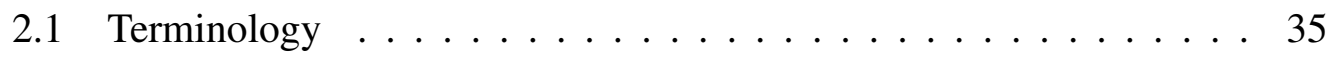

2.2 Human Inability to Distinguish Falsehood . . . . . . . . . . . 36

2.2.1 Psychological Factors . . . . . . . . . . . . . 37

2.2.2 Social Echo-Chamber . . . . . . . . . . . . 38

2.3 Related Works . . . . . . . . . . . . . . . . . . . . . 39

2.3.1 Claim Content-based Approaches . . . . . . . . . . . . . . . 39

2.3.2 Knowledge-based Approaches . . . . . . . . . . 42

2.3.3 Social Context-based Approaches . . . . . . . . . . . . . 44

$2.3 .4 \quad$ Recap . . . . . . . . . . . . . . . . 47

2.4 Complementary Research Areas . . . . . . . . . . . . . . . . 47

2.4.1 Check-Worthiness Prediction . . . . . . . . . . . 47

2.4 .2 Stance Detection . . . . . . . . . . . . . . . . . 49 
2.4 .3 Rumor Classification . . . . . . . . . . . . . . . . 50

2.4 .4 Truth Discovery . . . . . . . . . . . . . 51

2.4.5 Source Credibility Analysis . . . . . . . . . . . 52

2.5 Related Datasets . . . . . . . . . . . . . . . . . . . 54

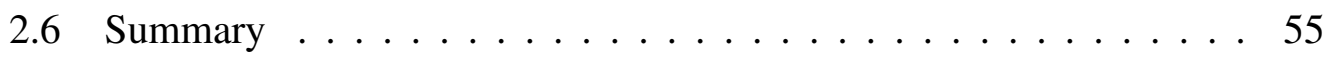

3 Background $\quad 59$

3.1 Deep Learning . . . . . . . . . . . . . . . . . . . . . . 59

3.1.1 Convolutional Neural Networks . . . . . . . . . . . . . 60

3.1.2 Recurrent Neural Networks . . . . . . . . . . . . . . . 61

3.1.3 Attention \& Self-Attention . . . . . . . . . . . . . . . . 62

3.1 .4 Latent Variable Model . . . . . . . . . . . . . . . 63

3.2 Temporal Point Processes . . . . . . . . . . . . . . . . . . . 64

3.2 .1 Poisson process . . . . . . . . . . . 65

3.2.2 Hawkes process . . . . . . . . . . . . 65

3.2 .3 Self-correcting process . . . . . . . . . . . 66

3.2 .4 Optimization ..................... 67

3.3 Evaluation . . . . . . . . . . . . . . . . 68

3.4 Summary ............................ 69

4 Hierarchical Stance Representation and Detection for Online News 71

4.1 Description of Stance Detection Task . . . . . . . . . . . . . . 71

4.2 Related Works . . . . . . . . . . . . . . . . . . 72

4.3 Hierarchical Classification . . . . . . . . . . . . 73

4.3.1 Two-Layer Neural Network . . . . . . . . . . . . . 73

4.3.2 Maximum Mean Discrepancy . . . . . . . . . . . . 75

4.3 .3 Optimization ................... 77

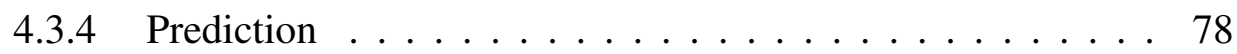

4.4 Experimental Setup $\ldots \ldots \ldots$. . . . . . . . . . 80

4.4.1 Research Questions . . . . . . . . . . . . . . . . . . . 80

4.4 .2 Datasets ...................... 81 
4.4 .3 Evaluation Measure . . . . . . . . . . . . . . . . . 81

4.4 .4 Feature Extraction $\ldots \ldots \ldots \ldots$. . . . . . . 82

4.4.5 Experimental Setting . . . . . . . . . . . . 82

4.4 .6 Baselines ..................... . 83

4.5 Results and Discussion $\ldots \ldots \ldots \ldots$. . . . . . . . . 84

4.5.1 Overall Performance $(\mathrm{RQ} 1) \ldots \ldots \ldots$. . . . . . 84

4.5.2 Dependency Assumption (RQ2) . . . . . . . . . . 87

4.5.3 Hyper-parameter Sensitivity (RQ3) . . . . . . . . . . . 88

4.5.4 Feature Analysis (RQ4) . . . . . . . . . . . . . . . . 89

4.6 Discussion . . . . . . . . . . . . . . . . . 90

4.6.1 Hierarchy and MMD . . . . . . . . . . . . . 90

4.6.2 Model Deployment . . . . . . . . . . . . . . . . . 92

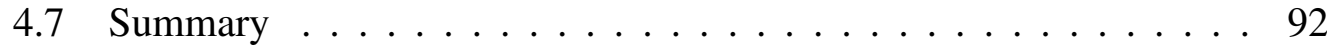

5 User Replies Aided Misinformation Detection 95

5.1 Problem Statement . . . . . . . . . . . . . . . . . 96

5.2 Motivation . . . . . . . . . . . . . . 96

5.3 Deep Latent Variable Model . . . . . . . . . . . . . . . . . . . . . 98

5.3.1 Encoding a Claim . . . . . . . . . . . . . . . 98

5.3.2 Encoding People's Replies . . . . . . . . . . . . . . . 100

5.3 .3 Veracity Modeling . . . . . . . . . . . . . . . . 101

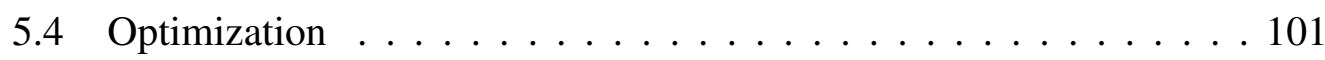

5.4.1 Inference Model . . . . . . . . . . . . . . . . . . . . 102

5.4 .2 Objective Function . . . . . . . . . . . . . . . . 103

5.4 .3 Gradient Estimation $\ldots \ldots \ldots \ldots$

5.4 .4 Prediction ...................... 105

5.5 Experiment Setup $\ldots \ldots \ldots 105$

5.5.1 Research Questions . . . . . . . . . . . . . . . . . . 105

5.5 .2 Datasets ......................... 105

5.5.3 Evaluation Measures . . . . . . . . . . . . . . . 107

5.5.4 Hyperparameters Setting . . . . . . . . . . . . . 107 


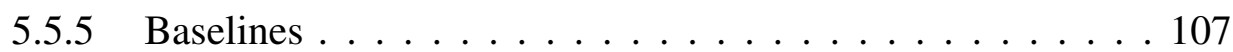

5.6 Results and Discussion . . . . . . . . . . . . . . . . 109

5.6.1 Performance Comparison (RQ1) . . . . . . . . . . 109

5.6.2 Ablation of the Latent Distribution (RQ2) . . . . . . . . . 111

5.6.3 Ablation of People's Replies (RQ3) . . . . . . . . . . . 112

5.6.4 Random vs. Temporal Ordered Replies (RQ4) . . . . . . . 112

5.6.5 A Latent Distribution for Replies (RQ5) . . . . . . . . . . . 113

5.6.6 Sensitivity Analysis (RQ6) . . . . . . . . . . . . . . . 114

5.7 Discussion . . . . . . . . . . . . . . . 115

5.7.1 Stance Detection and Veracity Evaluation . . . . . . . . . 115

5.7.2 Probabilistic Modeling and Uncertainty . . . . . . . . . 116

5.7.3 Model Deployment . . . . . . . . . . . . . . 117

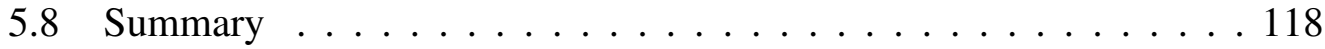

6 Self-Attentive Hawkes Process 119

6.1 Introduction . . . . . . . . . . . . . . . . . . . . . . 119

6.2 Related Work . . . . . . . . . . . . . . . . . . . . . 122

6.3 Self-Attentive Hawkes Process . . . . . . . . . . . . . . . . . 123

6.4 Experiments . . . . . . . . . . . . . . . . 126

6.4.1 Research Questions . . . . . . . . . . . . . . . . . 126

6.4 .2 Synthetic Dataset . . . . . . . . . . . . . . . 127

6.4.3 Real-World Datasets . . . . . . . . . . . . . . . . 128

6.4 .4 Training Details . . . . . . . . . . . . . . 130

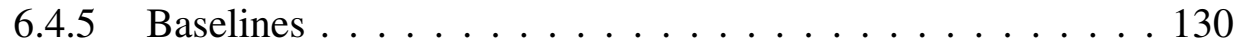

6.5 Results and Discussion $\ldots \ldots \ldots 131$

6.5.1 Goodness of fit on the synthetic dataset (RQ1) . . . . . . 131

6.5.2 Overall Performance (RQ2) . . . . . . . . . . . . 132

6.5.3 Number of samples' influence (RQ3) . . . . . . . . . . . . 134

6.5.4 Computational Efficiency (RQ4) . . . . . . . . . . . . 134

6.5.5 Model interpretability (RQ5) . . . . . . . . . . . 134

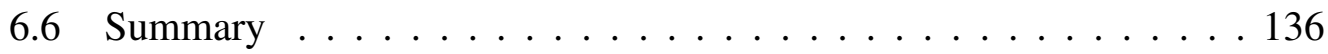


$7 \quad$ Engagement Dynamics via Hawkes Processes 137

7.1 Problem Formulation . . . . . . . . . . . . . . . . 137

7.2 Hybrid Model Description . . . . . . . . . . . . . . . . . 140

7.2.1 Engagement by Temporal Point Processes . . . . . . . . . . . 140

7.2.2 Predictive Task . . . . . . . . . . . . . . . . 141

7.2.3 Veracity Classification . . . . . . . . . . . . . . 142

7.3 Experiments . . . . . . . . . . . . . . . . . 143

7.4 Results and Analysis . . . . . . . . . . . . . . . . . . . . . . . 144

7.4.1 Misinformation Classification Performance (RQ1) . . . . . 144

7.4 .2 Time Prediction $(\mathrm{RQ} 2) \ldots \ldots \ldots \ldots$

7.5 Discussion of Model Deployment . . . . . . . . . . . . . . . . . . 144

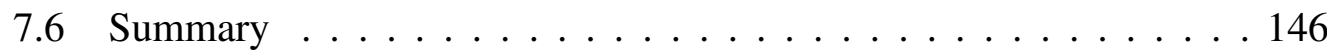

8 Conclusions \& Future Work $\quad 147$

8.1 Main Findings . . . . . . . . . . . . . . . . . . 147

8.2 Limitations . . . . . . . . . . . . . . . . . . . . . . . . 149

8.3 Future Work . . . . . . . . . . . . . . . . . . . . . . 150

8.3.1 Overcoming Limitations . . . . . . . . . . . . . . . . 150

8.3.2 Expanding Research . . . . . . . . . . . . . . 151

$\begin{array}{lr}\text { Appendices } & 152\end{array}$

$\begin{array}{ll}\text { A Full List of Publications } & 153\end{array}$

$\begin{array}{ll}\text { Bibliography } & 154\end{array}$ 



\section{List of Figures}

1.1 An example of a false claim and people's replies to it. From the replies, 123 users chose to trust the claim and re-tweeted it as if it was true, and 20 users liked this post. . . . . . . . . . . 26

1.2 False information is impactful as it (a) survives for a long time, and (b) is viewed by thousands of people. This figure is originally

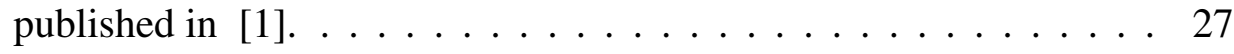

1.3 The overview of the section structure of this thesis. . . . . . . 33

2.1 Social media platforms can produce echo-chambers, which lead to polarization and can encourage the spread of false information. This figure shows echo-chambers formation in retweet graph on controversial \#beefban topic. This figure is originally published in [2]. . . 38

2.2 The fact-checking pipeline: 1 . check-worthiness estimation, 2. verified claim retrieval, and 3. fake news detection. This figure is originally published in [3] . . . . . . . . . . . . . . 43

2.3 Mind map of the features and models for veracity evaluation. . . . . 48

2.4 A diagram that shows how the listed research areas link to each other. 53

3.1 An example of the CNN model, LeNet-5, to classify characters. This figure is originally published in $[4] \ldots \ldots$. . . . . . . 60

3.2 A recurrent neural network and the unfolding in time of the computation involved in its forward computation. This figure is originally published in [5]. . . . . . . . . . . . . . . . 6 
3.3 An illustration of self-attention. This figure is originally published in [6].

3.4 An illustration of VAE. This figure is originally published in [7]. . . 64

3.5 An example of the point process realization and it counting process. This figure is originally published in [8]. . . . . . . . . . . 65

3.6 An example of the intensity function of an Hawkes process. This figure is originally published in $[8] \ldots \ldots \ldots 6$

4.1 The architecture of our proposed two-layer neural network. . . . . 73

4.2 The confusion matrices of our model for the augmented Emergent (on the left) and FNC-1 (on the right) datasets. . . . . . . . . . . 86

4.3 t-SNE visualization of the hidden representations on the training data. Blue points denote hidden representations of unrelated samples from the first layer while red points denote those of stance samples from the second layer. The curves indicate the distribution of data points when projected to the one-dimensional space (the $x$-axis and the $y$-axis). The hidden representations of model trained (a) with separated layers (b) together but without regularization, and (c) with MMD regularization. . . . . . . . . . . . . . . 88

4.4 Sensitivity of the trained model when varying $\alpha$ and $\beta$ on the test subset of the augmented Emergent (on the left) and FNC-1 (on the right) datasets.

5.1 An example of a false claim and people's replies to it. From the replies, 231 users chose to trust the claim and re-tweeted it as if it was true, while only 4 users asked for further clarifications. . . . . . 97 
5.2 The Framework of the deep latent variable model. The framework consists of two major parts, the claim encoder ( $\$ 5.3 .1)$ and the replies encoder ( $\$ 5.3 .2)$, the concatenation of which determines the claim veracity. Blocks and nodes represent computation modules and variables. Grey nodes are observed variables while blank nodes are latent variables (similarly with Figure 5.3). Note that blocks of the same color denote the same module. . . . . . . . . . . . . . 98

5.3 The directed graphical model. Grey nodes represent observed variables while blank nodes represent latent variables. Solid lines denote the generative model $p_{\theta}\left(z \mid h_{c}\right) p\left(y \mid z, h_{\mathfrak{D}}\right)$, dashed lines denote the variational approximation $q_{\phi}\left(z \mid y, h_{c}, h_{\mathfrak{D}}\right)$ to the intractable posterior $p_{\theta}\left(z \mid h_{c}\right)$. The variational parameters are learned together with the generative model parameters. . . . . . . . . . . . . . . 102

5.4 Example data instances from the Pheme dataset that are correctly predicted in Figure (a) and mis-predicted in Figure (b). . . . . . . 110

5.5 The impact of the latent distribution $p$ on the model performance. In both figures we show the performance change on all the evaluation measures of the model with (Full) or without (Ablated) $p$. Figure (a) shows it for the RumourEval test subset and Figure (b) shows it for the Pheme test subset. . . . . . . . . . . . . . . . . . . 11

5.6 The impact of people's replies on the model performance. In both figures we show the performance change on all the evaluation measures of the model with (Full) or without (Ablated) replies information. Figure (a) shows performance for the RumourEval test subset Figure (b) shows it for the Pheme test subset. . . . . . . . . . . . . . 112

5.7 The effect of the temporal order of the reply encoder on model performance. In both figures we show the performance change on all the evaluation measures of the model with random and temporal ordered people's replies. Figure (a) shows it for the RumourEval test subset and Figure (b) shows it for the Pheme test subset. . . . . . . . 113 
5.8 The effect of an additional latent distribution for people's replies on the model performance. In both figures we show the performance change on all the evaluation measures of the model with (Expanded) and without (Full) an additional latent variable for the people's replies. Figure (a) shows it for the RumourEval test subset and Figure (b) shows it for the Pheme test subset. . . . . . . . . . 114

5.9 The effect of the latent variable dimension on model performance. In both figures we show how the accuracy and $F_{1}$ scores change when varying the dimension of $z$. Figure (a) shows it for the RumourEval test subset and Figure (b) shows it for the Pheme test subset. . . . . . . . . . . . . . . . . . 115

6.1 Three users on social media platforms exert different types of actions. The filled dark symbols in (a) mean four action types while the red arrows denote actions influencing another actions. A $\checkmark$ symbol in the cell $(i, j)$ in (b) indicates the influence of the column event type $j$ on the row type $i$ on future events. . . . . . . . . 121

6.2 An event stream and the SAHP for one event type $(u)$. The intensity function $\lambda_{u}(t)$ is determined by a sequence of past events via the SAHP. . . . . . . . . . . . . . . . . . . . . . . 124

6.3 The time-shifted position embedding of an event with $i=14$ in a sequence. Squares and diamonds denote conventional and new embedding values with time shifts. . . . . . . . . . . . . . . . 124

6.4 The four triggering kernels of the synthetic dataset with 2 event

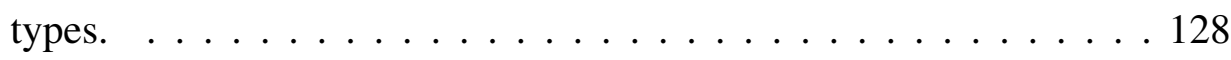

6.5 The intensities of the two-dimensional Hawkes processes over the synthetic dataset. The upper and the lower subfigure correspond to the dimension-1 and the dimension-2 respectively. 
6.6 QQ-plot of true VS estimated intensities of types. The x-axis and the $y$-axis represent the quantiles of the true and estimated intensities. For each model the top figure is for the type-1 while the bottom figure is for the type- 2 events. . . . . . . . . . . . . . . 131

6.7 The influence of the number of samples of the Monte Carlo estimation on SAHP's performance for event prediction. . . . . . . . . . . 134

6.8 Expected attention weights among event types on the StackOverflow test set. . . . . . . . . . . . . . . . . . . . . 135

7.1 Graph representation of source claim dissemination tree, where nodes represent interaction events such as comments and retweets. . 139

7.2 An illustrative scheme of the engagement graph by temporal point processes. . . . . . . . . . . . . . . . . . . . . 140 



\section{List of Tables}

2.1 This table categorizes research on false information based on the platforms they study. . . . . . . . . . . . . . . . . . 56

2.2 This table categorizes research based on the characteristics and features of false information analyzed. . . . . . . . . . . 57

4.1 Statistics of the datasets. . . . . . . . . . . . . . . 81

4.2 Performance comparison of our model against the State-of-the-Art models on the augmented Emergent dataset. . . . . . . . . . . 85

4.3 Performance comparison of our model against the State-of-the-Art models on the FNC-1 dataset. . . . . . . . . . . . . . . . . 85

4.4 Performance of our model with different feature sets on the FNC-1 dataset. "/" denotes no feature set is removed. . . . . . . . . . . . . 89

5.1 Statistics of the datasets. . . . . . . . . . . . . . . 106

5.2 Performance comparison of the proposed deep latent variable model against the baselines. . . . . . . . . . . . . . . . . . . . 109

6.1 The symbols used throughout the paper . . . . . . . . . . . 123

6.2 Statistics of the used datasets. . . . . . . . . . . . . . . . . 127

6.3 Negative $\log$-likelihood per event on the four test sets. . . . . . . . 132

$6.4 F_{1}(\%)$ of event type prediction on the four test-sets. . . . . . . . 133

6.5 RMSE of event timestamp prediction on the four test sets. . . . . . 133

6.6 Model running time on the retweet dataset with a Titan Xp GPU card.135

7.1 Description of the notations used in this paper. . . . . . . . . . . 139 
7.2 Statistics of the Twitter15 and Twitter16. . . . . . . . . . . . 143

7.3 Test results on the experimental datasets. . . . . . . . . . . . 145 


\section{Chapter 1}

\section{Introduction}

“... news writers who, without any regard to truth or to what should be like truth, invent and put into the papers whatever might serve the ministers. This suffices with the mass of the people who have no means of distinguishing the false from the true paragraphs of a newspaper".

-Thomas Jefferson (Oct. 13, 1785)

Jefferson's viewpoints are as salient today as they were in 1785. Some 236 years later, there are still a "mass of the people" struggling to distinguish between true and false news contents. The growing web technology has amplified the adverse impacts of this struggle.

\subsection{Background \& Motivation}

The web and social media give birth to highly interconnected world-wide platforms. Common examples are social media sites and news service providers, including Facebook, Twitter, microblog and news feeds. Moreover, digital media is serving as an increasingly major source of news for the public $[9,10]$. A recent study from Pew Research shows that $49 \%$ of USA adults received news from social media in 2012 and this number climbed to $68 \%$ in $2018{ }^{1}$. Compared to traditional newspapers, digital news is (i) more time-efficient and (ii) easier to share or interact with other consumers. Therefore, words in digital news exert an increasingly considerable effect in shaping the public beliefs and awareness.

\footnotetext{
${ }^{1}$ www. journalism.org/2018/09/10/news-use-across-social-media-platforms-2018/
} 


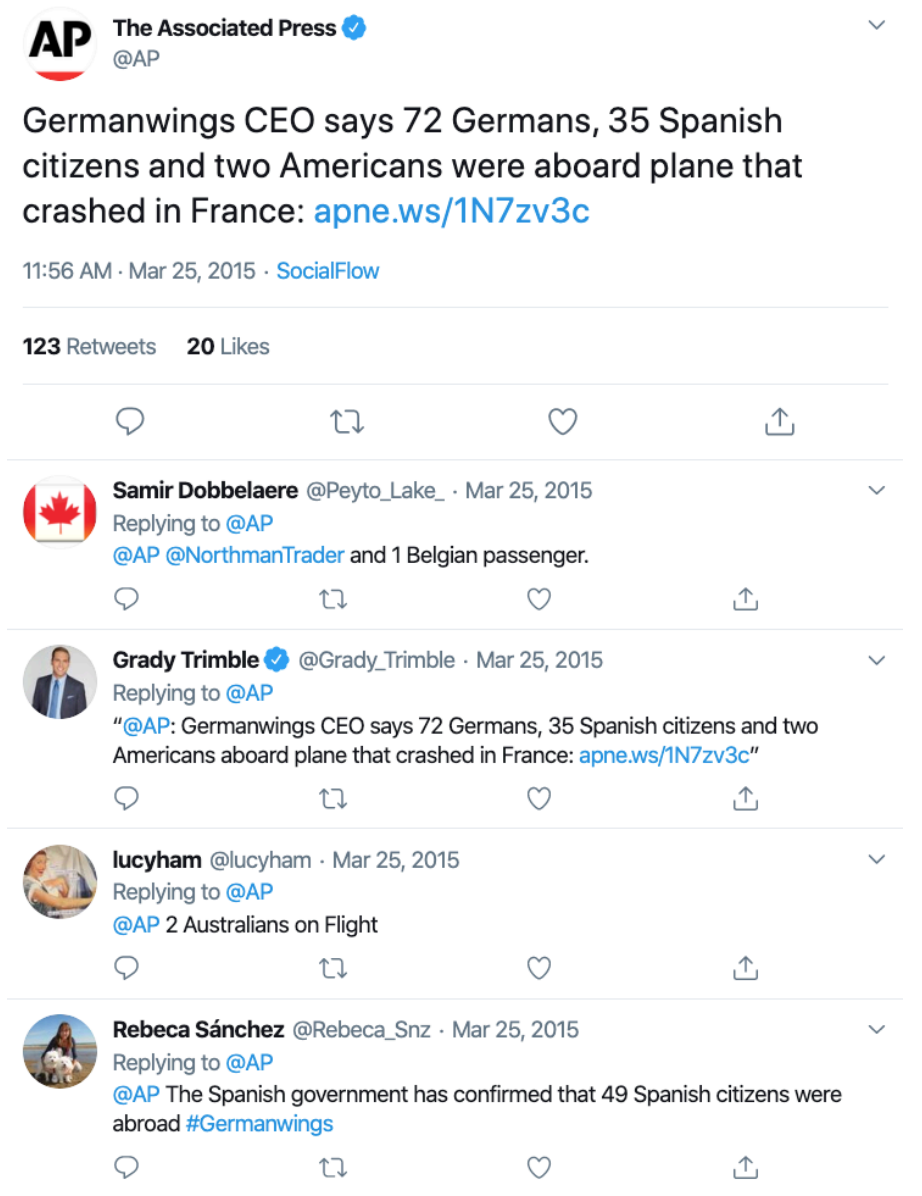

Figure 1.1: An example of a false claim and people's replies to it. From the replies, 123 users chose to trust the claim and re-tweeted it as if it was true, and 20 users liked this post.

Due to the absence of careful checking, the web and social media platforms are fraught with false content from malicious agents. Though false information has existed for centuries - news writers used it to manipulate the public as Jefferson wrote- its popularity and propagation on social media platforms amplifies its threat to traditional journalistic norms and the general public [11]. Figure 1.1 shows a inaccurate claim posted on Twitter about an airplane accident. It starts with a tweet stating a false claim, and is followed by a branch of replies towards the claim from social users. Replies typically express stances of authors discussing the veracity of the claim. For example a reply showed more information to make it more accurate. However, more consumers believed the claim and re-tweeted it as if it was true. 

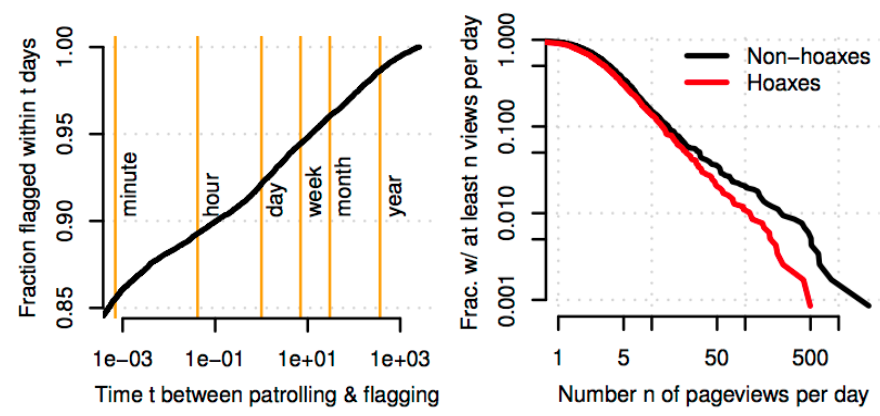

Figure 1.2: False information is impactful as it (a) survives for a long time, and (b) is viewed by thousands of people. This figure is originally published in [1].

Various works have reported quantitative measurement of the impacts of online false information $[1,11,12]$. Regarding the measurement metrics, these works selected the statistics on engagements that a piece of false information generates on social media and the web. For instance, researchers use the times of consumer readings, the number of consumers it reaches via re-sharing and the temporal duration (e.g., days) it exists before it is removed. It is concluded by these works that popular misinformation pieces propagate more widely and attract a larger population of consumers, as shown in Figure 1.2.

Wide-spreading false information causes growing concern among the public. It disturbs political elections [11], manipulates individuals' awareness [13, 14], polarizes people's opinions, affects financial markets [15], threatens public health, and delays responses during disasters [16]. One well-known piece of false information is "Pizzagate" 2 which produced over 1 Million Twitter posts at the end of the 2016 US presidential election [17]. Additionally, one German official affirmed in 2017 about "investigating an unprecedented proliferation of fake news items amid reports of Russian efforts to influence the country's election later in this year" 3 . More recently, a video named "Plandemic" on Youtube ${ }^{4}$ shared false information about COVID-19 that severely blunted response to the pandemic. Wide influence of false information makes the web a battlefield.

\footnotetext{
${ }^{2}$ https://en.wikipedia.org/wiki/Pizzagate_conspiracy_theory

${ }^{3}$ https: / / www. theguardian.com/world/2017/jan/09/ germany-investigating-spread-fake-news-online-russia-election

${ }^{4}$ https: / / www. nytimes.com/2020/05/20/technology/ plandemic-movie-youtube-facebook-coronavirus.html
} 
The far-reaching negative impacts of widespread false information on the public highlights the urgency of evaluating the veracity of online textual claims. The difficulty comes in part from a human inability to accurately distinguish truth from falsehood. Studies showed that when given a fake news article, people believed it "somewhat or very accurate $75 \%$ of the time" [18]. Consequently, manual fact checking emerges with the promise to rectify this epidemic of false textual claims. A valuable attempt has been made by some third-party organizations such as: Snopes ${ }^{5}$, and Emergent ${ }^{6}$. These organizations employ professional journalists to manually check the veracity of claims made by organizations and public figures. The fact checkers must refer to previous reports and known facts, combined with reasoning to reach a verdict. Such a manual approach is very expensive and too slow to be able to check all the daily generated textual claims on the web. Thus, automatic tools are in great demand to speed up this verification process.

\subsection{Research Challenges}

With the above mentioned motivations, this thesis sets out to explore data-driven machine learning models to evaluate the veracity of textual claims on the web. A textual claim denotes a piece of words whose content is of interest to the public and can potentially exert negative influence on a group of people [19]. Veracity evaluation is defined as the task of classifying the truthfulness of a textual claim into one of the following categories: True and False (an additional category Unverified can be considered in other works).

False information exists widely in almost all information dissertation platforms, including news websites and social media like Twitter. To be specialized, this thesis studies false information with two application contexts: online news in Chapter 4 and social media in Chapter 5, 6 and 7. Correspondingly, claims are instantiated by news headlines in Chapter 4 and social media posts in other chapters. Veracity evaluation on other platforms, such as crowdsourced Wikipedia or structured knowledge graphs, is out of the scope of this thesis.

\footnotetext{
${ }^{5}$ https: //www. snopes.com/

${ }^{6}$ http $: / /$ ww . emergent. info/
} 
The task of automated veracity evaluation is one rife with challenges. We analyse and discuss major challenges that we tackle in the thesis.

- Stance detection is an important first step towards veracity evaluation. We study the stance detection task in the context of online news, because it simplifies veracity evaluation on the premise that supportive stances from reputed articles can prove claim truthfulness. Previous methods tackle stance detection in online news as a multi-class classification problem, i.e., classifying agree, disagree, discuss, and unrelated, which neglects the semantically hierarchical structure in the four stance classes. Also, there is often severe class imbalance in the stance datasets [20]. Consequently, commonly-used four-way classifiers fail to correctly classify instances that fall into minority classes, thus suffering from deficient performance.

- Beyond news articles, false information widely spreads in social media. Social users often express their stance by writing comments and replies that are important evidential signals. Hence, we also study how to utilize user replies to infer claim veracity, where user stances are indirectly considered. The major challenge is that claims and user replies are usually short and can be ambiguous [21]. There may be mixed objective description and misleading information. Previous works are mostly deterministic and cannot properly deal with the in-built semantic ambiguity and fail to capture salient credibility representation. It is thus necessary to develop a more effective way of utilizing user replies in social media.

- The above content-based models do not consider the dynamics of user engagement in misinformation spreading. Recent models use static graph neural networks to extract geometric patterns; others leverage time-series analysis by treating misinformation spread as a temporal event sequence [22, 23]. These two approaches each neglect the alternative engagement structure with neither leveraging both geometric and temporal dissemination features. Effective models of engagement dynamics need further investigation. 
In addition to the these challenges, it is essential to discuss practical issues when deploying machine learning models in a real-world scenario. First of all, veracity evaluation systems cannot be used on their own and that human fact checkers are needed for giving the final verdict on the veracity of a given statement. It is also very important for automated systems to provide an explanation, rationale, on why they have assigned a certain category. Secondly, it is necessary to pay attention to model efficiency in terms of time complexity and space storage requirement. A large number of claims are generated in news articles and social media platforms on a daily basis, while veracity evaluation is time-critical and requires swift actions in detecting and removing online misinformation. Thus, veracity evaluation models need to deal with these daily generated claims in an efficient way. The third issue is to consider how often a machine learning model should be re-trained and updated as more data are available, so as to avoid bias from the used datasets and the way data is used by the model. Such biases may make the social platforms favoured or against certain user accounts and the way of utterance. In following chapters, we will give more detailed discussion of how each proposed model can be deployed and what issues need to be addressed during deployment.

\subsection{Major Contributions}

In this section we summarize the main contributions of this thesis. The works presented in this thesis sit at the crossing between the fields of machine learning, natural language processing and social network analysis. Our works come in the forms of analytical, algorithmic and empirical contributions.

\subsubsection{Hierarchical Stance Detection}

We chose to study the stance detection task in the context of online news, because it simplifies veracity evaluation on the premise that supportive stances from reputed articles can prove claim truthfulness. Other contexts, such as social media conversations, usually involves users' instant reactions without careful investigations. Hence stance in these contexts alone cannot be reliably used to identify falsehood, but usually acts as an auxiliary signal in a multi-task setting. 
In the context of online news articles, the stance detection task aims to categorise the stance of an article towards a claim into four classes: agree, disagree, discuss and unrelated. Since agree, disagree and discuss can be assumed as related, we analyse that there is a semantically hierarchical structure among the four stance classes. Therefore, classification of related/unrelated is a different task from that of agreeldisagreeldiscuss.

In Chapter 4, we propose a two-layer neural network based on this analysis. The first layer aims at identifying relatedness, while the second layer aims at classifying, those identified as related, into the other three classes: agree, disagree and discuss. We improve state-of-the-art performance of the stance detection task. Moreover, by studying various levels of dependency assumptions between the two layers: (1) independent, when there is no error propagation between the two layers; (2) dependent, when the error propagation is left free, and; (3) learned, when the error propagation is controlled by Maximum Mean Discrepancy, we show that when learned, the model (a) better disentangles the distributions of related and unrelated stances and (b) outperforms the state-of-the-art accuracy for stance detection.

\subsubsection{User Replies Aided Misinformation Detection}

Besides news articles, people often express their stance by writing comments and replies in social media. Hence user replies in social media can provide evidential signals for newly emerging claims. To identify falsehood, existing models produce deterministic mappings from claims and people's replies to veracity predictions. One limitation is their inability to represent ambiguity of semantics. Chapter 5 presents a deep latent variable model that incorporates stochastic factors to semantic distribution and obtain more salient credibility features for veracity evaluation.

The model consists of three modules. First, we employ a Bidirectional Long Short Term Memory (BiLSTM) to extract textual features from claims, based on which we compute a latent distribution. Second, we use the same BiLSTM to encode the single reply and a LSTM to summarize all the replies of the claim in temporal order. Finally, the model computes the veracity prediction with the reply embedding and the sample from the latent distribution. To train the proposed 
deep latent variable model, due to the analytical intractability of the posterior distribution, we develop a Stochastic Gradient Variational Bayes (SGVB) algorithm. By marginalising over latent variables, the model is more robust against overfitting, which is essential for the relatively small-sized misinformation datasets.

\subsubsection{Detection and Forecast via Engagement Dynamics}

The above-mentioned models focus on the content of claims and news articles/social user replies. To extend these content-based models, we study the temporal dynamics of user engagement in misinformation on social media platforms. Specifically, engagement-based misinformation analysis makes use of patterns that can be attributed to the dynamics of spread. Specifically, we formulate the user engagement in misinformation as a dynamic graph with timestamped events, with each event containing engaged user profiles and textual content such as replies. We develop a Temporal Point Process (TPP) model to extract the temporal evolution patterns and geometric features of the graph. This work can be seen as an extension of user engagement-based veracity evaluation from purely textual information to a hybrid of textual, temporal and geometric features.

In Chapter 6, to improve the capability of the vanilla TPP in capturing temporal dynamics, we propose Self-Attentive Hawkes Process (SAHP) that incorporates self-attention to measure the influence of history events on the occurrence of subsequent events. One deficit of the conventional self-attention, when applied to event sequences, is that its position encoding only considers the order of a sequence ignoring the time intervals between events. To overcome this deficit, we modify position embeddings by translating time intervals into phase shifts of sinusoidal functions.

In Chapter 7, we build a dynamic engagement graph where user engagements are nodes. We view the addition of new engagement nodes as the chronological events. To extract both geometric and temporal features of this dynamic graph, we modify the SAHP model to generate the graph embedding. Claim veracity evaluation is converted to the the task of engagement graph classification. Further, we model user engagements in the temporal network, so as to forecast the future rate of spread for a claim identified as misinformation. 


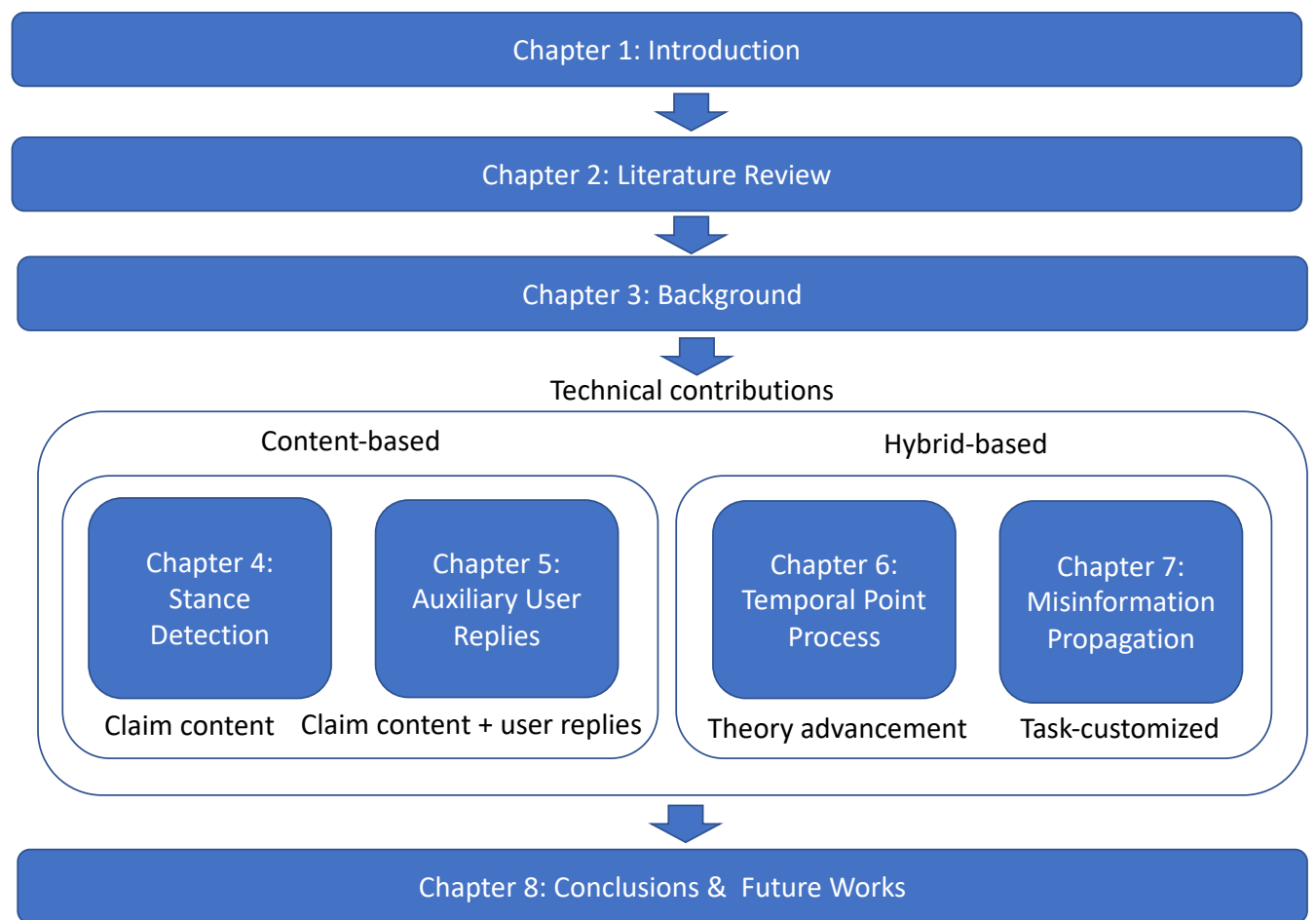

Figure 1.3: The overview of the section structure of this thesis.

\subsection{Thesis Overview}

This section provides an overview of this thesis in Figure 1.3.

- Chapter 1, to which this section belongs, gives an introduction to the subject of this thesis. This chapter also provides an overview of the research questions, the major challenges and the contributions.

- Chapter 2 then reviews the literature including terminology, related works and complementary research areas.

- Chapter 3 presents the preliminary mathematical background for the understanding of the following technical chapters in this thesis.

- Chapter 4 is about news stance detection towards the claim being evaluated. A hierarchical detection model is proposed and examined with three settings.

- Chapter 5 describes a deep latent variable model that allows multimodal semantic distribution and extracts salient information from people's replies on social media platforms. 
- Chapter 6 presents how we leverage the self-attention mechanism to modify temporal point processes.

- Chapter 7 is an application of the model proposed in Chapter 6 for the task of misinformation detection. We also show how to forecast the growth of the engagement network.

- Chapter 8 concludes this thesis, where we summarize the content and findings of this thesis and briefly reflect onto future work.

\subsection{Supporting Publications}

The selected publications which support the chapters of this thesis are listed as below. The full publication list is available in Appendix A.

Chapter 4 Q Zhang, S Liang, A Lipani and E Yilmaz. From Stances' Imbalance to Their Hierarchical Representation and Detection. WWW, San Francisco, 2019.

Chapter 5 Q Zhang, A Lipani, S Liang and E Yilmaz. Reply-Aided Detection of Misinformation via Bayesian Deep Learning. WWW, San Francisco, 2019.

Chapter 6 Q Zhang, A Lipani, O Kirnap and E Yilmaz. Self-Attentive Hawkes Process. ICML, Austria, 2020.

Chapter 7 Q Zhang, J Cook and E Yilmaz. Detecting and Forecasting Misinformation via Temporal and Geometric Propagation Patterns. ECIR, Norway, 2021. 


\section{Chapter 2}

\section{Literature Review}

In this chapter, we present the literature and relevant works that serve as a basis for this thesis. In Section 2.1, we start with definitions and distinctions of key terminologies, then Section 2.2 provides the analysis of human inability to distinguish false information with psychological and social causes. We continue with the literature review on computational veracity evaluation methods in Section 2.3 In Section 2.4, we describe areas that are strongly relevant to the research problem of this thesis. Two tables summarize typical veracity evaluation methods on different platforms in Section 2.5.

\subsection{Terminology}

False information originally is new articles with false claims or statements. It exists in various forms and by different names on the web. To avoid confusion, we hereby provide definitions and distinctions on key terminologies that are strongly relevant to false information.

- Rumor is a type of "information or story that is passed from person to person but has not been proven to be true" 1 that makes an ambiguous picture of facts. The truthfulness value of a rumor can be: True, False, or Unverified, as studied in $[24,25]$.

- Misinformation is defined as "information that is not true or accurate" 2 [26].

\footnotetext{
${ }^{1}$ https://www.merriam-webster.com/dictionary/rumor

${ }^{2}$ https: //www.merriam-webster.com/dictionary/misinformation
} 
- Disinformation refers to "false information deliberated and often covertly spread in order to influence public opinion or obscure the truth" 3 [26].

- Fake news is defined as "false stories that appear to be news, spread on the internet or using other media, usually created to influence political views or as a joke" 4 . It is usually seen as a subset of disinformation that appears to be genuine news articles [26].

- Satire news with adequate context "has no intent to mislead or deceive readers and can hardly be mis-perceived as factual" [17].

- Hate speech aims ar the use of emotive and abusive language instead of veracity evaluation [27].

We make two further declarations. (i) Rumors including long-term ones, such as conspiracy theories, as well as short-term emerging ones may not be properly verified [28]. While misinformation refers to pieces of information relevant to public news events that can be verified as false. (ii) Compared to misinformation, disinformation additionally assumes malicious motives to mislead readers and thus is considered a subset of misinformation [26]. This thesis aims to detect misinformation but does not distinguish it from disinformation.

\subsection{Human Inability to Distinguish Falsehood}

False information would not be able mislead the public if consumers could distinguish the falsehood from truth. It is therefore interesting to look into the reasons of human beings' susceptibility. Several works [1,29-31] find that people often confuse the truth from falsehood: fake articles with adequate references are mistaken to be true while true articles with few references are mistaken to be fake. This finding suggests that human beings give high emphasis on the appearance when judging the veracity. One potential danger is that, if false information is generated intentionally to look genuine, humans are quite easy to be deceived.

\footnotetext{
${ }^{3}$ https: / / www.merriam-webster.com/dictionary/disinformation

${ }^{4}$ https: / / dictionary. cambridge.org/dictionary/english/fake-news
} 
What make things worse is that, with the advent of deep learning, false information might be generated by machines. When intentionally fine-tuned for specific purposes, it can be as deceptive as that generated by human beings. One research work has confirmed that humans can easily misjudge false information when it is created purposely to look like true information by machine learning algorithms [31].

We give an analysis of the reasons of human inability to discern false information based on psychological and sociological theories.

\subsubsection{Psychological Factors}

Psychological factors considerably influence humans' vulnerability to false information on the web. Two of the most influential factors are naïve realism and confirmation bias [17]. Naïve realism means that people believe they have the true awareness of facts whereas opposing opinions are not accurate or even false [32]. Confirmation bias is defined as the propensity of humans to look for information pieces that agree with their pre-existing cognation [33]. This type of bias forces people to seek evidence, not matter of veracity, that verifies their own claims. This can be exemplified by the cases that conservative consumers tend to read posts and news articles that hold the conservative standpoints, and vice versa. Further, the social normative theory indicates that humans share information pieces confirming their peers' viewpoints, to gain acceptance and social safety, regardless of the veracity of the shared content [34].

Mentality and demographics are another factors that play important roles in the success of false information in deceiving humans. It has been investigated that there is clearly a positive correlation between the tendency of critical thinking and the ability to distinguish falsehood from true information [35]. This also provides evidence that the wide propagation of misinformation is partially caused by the lack of critical and analytical thinking of news consumers. Moreover, improper understanding of the intension of the publishers might lead consumers to misjudge an information news [36]. Recent analysis of demographics [37] find that humans with higher education spend more time on digital news, and the senior have a more accurate perception of the reality. 


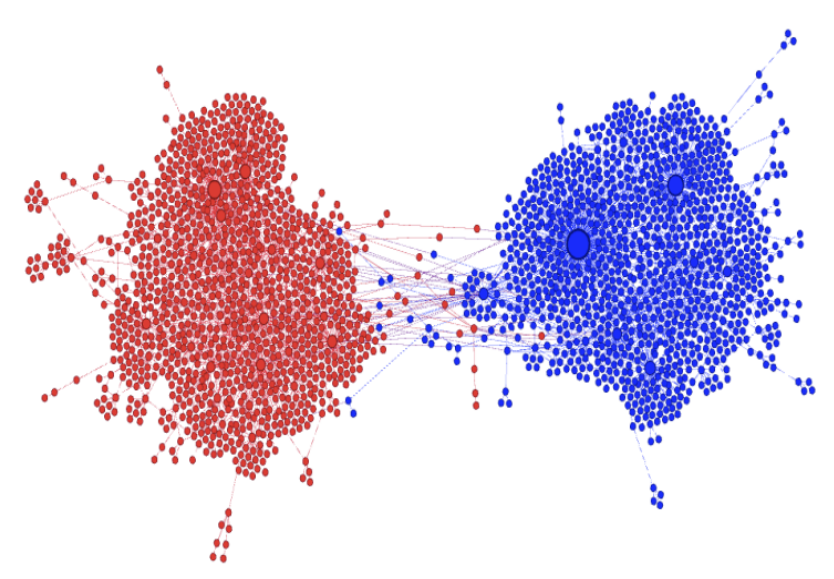

Figure 2.1: Social media platforms can produce echo-chambers, which lead to polarization and can encourage the spread of false information. This figure shows echochambers formation in retweet graph on controversial \#beefban topic. This figure is originally published in [2].

\subsubsection{Social Echo-Chamber}

The echo-chamber effect means the self-promotive polarizing phenomenon in a certain group of people who are surrounded by information confirming their preexisting beliefs. This effect has been observed to become severe, one reason of which is that recommendation algorithms intend to display same type of contents [38]. For instance, a political conservative might follow more conservatives on Facebook, like conservative-minded posts, and thus frequently be exposed to news articles that confirms her viewpoint. Figure 2.1 visualizes this echo-chamber effect on Twitter on a controversial topic of \#beefban, where red and blue nodes represent users with opposing beliefs and edges represent who-retweets-whom [2]. Notice that there are few message connections betwenn the two groups.

Several works have studied the roles of echo-chambers in the spread of misinformation. Quattrociocchi et al. [39] show that the echo-chamber effect, by polarizing humans' standpoints, make humans tend to believe what they are exposed to. This study also suggests that exposure to repeated content increases the tendency to accept the content, regardless of its veracity. Hence, one interesting direction is to develop efficient techniques to break echo-chambers among communities and diminish the propagation of false information. 


\subsection{Related Works}

In the previous section, we identify the issues that lead to humans' venerability of false information. Now, we review relative works of computational approaches for veracity evaluation.

\subsubsection{Claim Content-based Approaches}

Content is the most basic source of veracity cues. The content-based approaches can be applied to a variety of forms, including short social posts and long news articles. For news articles, there are a set of attributes that suggest veracity, such as authors, headlines, main body text and accompanied images and videos. These attributes might not exist in all the objects being verified. Researchers have employed textbased and multimedia-based approaches to distinguish true and false information.

\subsubsection{Text}

Textual claim classification needs training data from annotated claims with verdicts by professional journalists [40]. False information is fabricated to mislead readers and consumers for political or financial purposes. Often, false content is characterized as being distorted, opinionated, misleading or aim to incite confusion [41]. These characteristics require particular writing styles to tempt readers. For instance, the headline of fake articles are often eye-catching, known as "clickbait". Hence it is reasonable to exploit text-based misinformation detection approaches

Different types of textual claims have been studied in various contexts. For example, in Fake News Challenge ${ }^{5}$, claims are instantiated as news headlines and researchers have focused on document-level semantic feature extraction and stance comparison with claims [20], where we will present technical contributions in Chapter 4. More commonly, textual claims refer to a short sequence of words usually a sentence or occasionally a short paragraph. It is easier for short sentences to circulate on social media, and researchers investigate linguistic features at the sentence-level and word-level in this context $[42,43]$. These fine-grained linguistic features are also important in document-level claim studies.

\footnotetext{
$5_{\text {http: //www. fakenewschallenge.org/ }}$
} 
Linguistic features are important indicators for veracity evaluation. These features are categorized as lexical, syntactic and semantic. Lexical features can be both character-level and word-level, being unique words, prefix/suffix (various lengths possible), stemmed words and lemmatized words. Syntactic features are a bit higher-level, i.e., the sentence-level features, being parts-of-speech tagging [44]. For example, researchers tried to spot deception phases based on Deep syntax [42] and Rhetorical structure [43]. The Deep syntax model uses probabilistic context free grammar to convert sentences into syntax structure rules. These rules can be employed to capture the differences between truthful and deceptive sentences. Semantic features can be bag-of-words and n-gram. One study extracted the number of paragraphs in a news article, the number of external links and quoted words [45]. And misinformation is reported to have higher perplexity [46].

Objectivity indicators, usually at the word-level, are another important textual features for claim veracity evaluation, as false claims are reported to have a decreased objectivity. Typical examples include hyperpartisan and yellow-journalism. The former refers to the extremely supporting attitude towards a political party, which is found to have a strong correlation to misinformation [45]. The latter refers to articles with a propensity of eye-catching, emotional expressions, such as clickbait. These articles usually aim to utilize the deceptive titles to convey misleading information [47]. Some sentiment features, such as positive words (e.g., love, sweet), negating words (e.g., not, never), cognitive action words (e.g., cause, know) and inferring action words (e.g., maybe, perhaps), are reported to help detect rumour [48]. Rashkin et al. used the lexicon of Linguistic Inquiry and Word Count (LIWC) and estimated the usage of strongly and weakly subjective words [49].

With the advent of deep learning, it might be fruitful to try more advanced NLP models, such as sentence embeddings [50,51] and pre-trained language models like Bert [52] and GPT-3 [53]. For example, COVID-19 misinformation is reported to have higher perplexity than truthful statements according to language models [46]. Although language information could indicate whether a claim is factual or not [54], false sentences may also be looking credible. 


\subsubsection{Multimedia}

Apart from textual claim content, multimedia, such as images and videos, is another important source of features for misinformation detection. As we have characterized in Section2.2, online misinformation exploits the individual vulnerabilities of people and thus often relies on sensational or even fake images to provoke anger or other emotional response of consumers. Also, visual elements can strongly manipulate peoples' opinions and thus have been widely tried in propaganda ${ }^{6}$. For example, Deepfakes [55] employ deep learning methods to generate fake images and videos to convey misleading information.

The mainline of these research works aim to extract visual-based features with different machine learning methods to decide if an image or video is manipulated. Some are had-crafted, for example, one recent study extracted various types of visual and statistical features to evaluate veracity of news articles [56]. Visual features include clarity score, coherence score, diversity score, and clustering score while statistical features include image ratio, multi-image ratio, hot image ratio, long image ratio, etc. More methods employed deep learning applied to images and videos. A convolutional neural network is used to extract text and visual features simultaneously in [57]. Researchers applied self-learning to detect and localize image splices and decide whether an image is self-consistent, in order to identify fake imagery [58]. A multimodal variational auto-encoder is developed to extract visual features in fake news [59]. Recently, the attention mechanism has been studied. For example, Qian et al. [60] proposed a hierarchical multi-modal contextual attention network, where Bert and ResNet are used to represent the texts and images contained in a claim respectively.

This thesis focuses on text content and does not involve multimedia when designing veracity evaluation algorithms. This is mainly because multimedia is sometimes missing in some benchmark data samples and it is unfair to compare text and multimedia-based algorithms against solely text-based counterparts.

\footnotetext{
${ }^{6}$ https://www.wired.com/2016/12/photos-fuel-spread-fake-news/
} 


\subsubsection{Knowledge-based Approaches}

Besides content-based approaches, there are some research works that utilize external knowledge sources [61-63], which is termed as fact-checking. Fact-checking gives verdicts by "researching and identifying evidence, understanding the context of information and reasoning about what can be inferred from this evidence" [28].

This fact-checking technique is usually used to check a short sequence of words, such as a social post or a major claim in news articles. When applied to long news articles, there is a check-worthiness prediction module ahead to decide which sentence in an article is worthy to check, which is the first task in Figure 2.2. Expert-based fact-checking is a traditional and resource-demanding way that requires domain experts to look into relevant documents to identify claim veracity. For instance, PolitiFact ${ }^{7}$ and Snopes ${ }^{8}$ employ journalist to fact-check claims. Increasing efforts are denoted to an automated computational fact-checking system.

Computational-based fact-checking develops a scalable system with the reference to existing knowledge, as shown in Figure 2.2. Knowledge sources can be structured or unstructured. Structured knowledge is usually stored in knowledge graphs such as DBpedia and Google Relation Extraction Corpus. There are two ways of utilizing structured knowledge: (1) retrieving existing elements that contain information supporting or denying the claim being verified [64], and (2) employ the topology to predict how likely a claim can be expressed as a node in the knowledge graph [62]. Unstructured knowledge refers to factual documents and reference corpus, including Wikipedia [65] and the open web resources [66,67].

The second task in Figure 2.2 is another popular way: matching a claim with existing, previously fact checked ones. This reduces the task to sentence-level textual similarity, which for example is used in ClaimBuster [68], Truthteller by The Washington Post ${ }^{9}$ and Full Fact Live platform ${ }^{10}$. It is common to repeat the same

\footnotetext{
${ }^{7}$ https://www.politifact.com/

${ }^{8}$ https://www.snopes.com/

${ }^{9}$ https://www.knightfoundation.org/articles/ debuting-truth-teller-washington-post-real-time-lie-detection- $\backslash$ service-your-service-not-quite-yet

${ }^{10}$ https://fullfact.org/blog/2017/jun/automated-fact-checking-full-fact/
} 


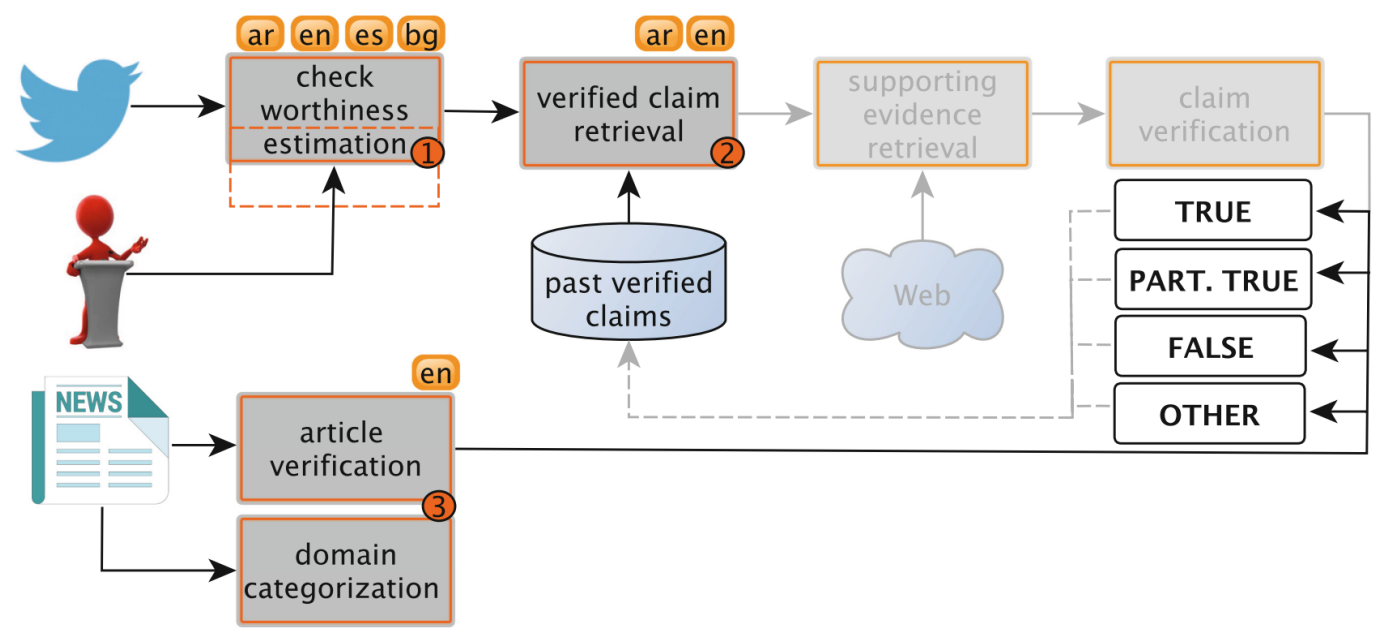

Figure 2.2: The fact-checking pipeline: 1 . check-worthiness estimation, 2 . verified claim retrieval, and 3. fake news detection. This figure is originally published in [3].

claims. However, this way cannot help with emerging claims.

Other ways to fact-checking have also been studied. Ferreira and Vlachos [69] convert fact checking to the task of Recognizing Textual Entailment (RTE) that aims to determine whether a hypothesis can justifiably be inferred from a premise [70-74]. The RTE-based models assume that the textual evidence to fact check a claim is given. Thus they are inapplicable in cases where this is not provided (as in HeroX), or it is a rather large textual resource such as Wikipedia (as in FEVER). For the latter, Thorne et al. (2018) developed a pipelined approach in which the RTE component is preceded by a document retrieval and a sentence selection component. There is also work focusing exclusively on retrieving sentence-level evidence from related documents for a given claim [75].

The growth of fact-checking has been hampered by the nature of the work. The main limitation is that verifying a claim needs extra knowledge, which is usually not contained in the claim itself but from an outside knowledge base. First, knowledge bases needs to be regularly updated, which is especially difficult in the case of newly emerging topics, such as the symptoms of COVID-19 in the early stages of the pandemic. Besides, NLP models trained on a certain corpus may have linguistic biases. Although this can be alleviated by structured data (subject, predicate, object), getting structured data is difficult. Therefore, ensuring the quality of the knowledge-base being used as ground truth is an additional challenge. 


\subsubsection{Social Context-based Approaches}

Social context refers to engagements of news consumers during the propagation process over time. It provides rich auxiliary features from a variety of perspectives to determine the veracity of claims. These approaches are designed in the context of social media platforms so the claims are usually short social posts. Commonly, we can categorize approaches based on social context as three types: users, replies, and networks.

\subsubsection{User-based}

User-based features are extracted from the user accounts that have interactions with or directly participate in the propagation process of false information. As misinformation is probably generated and spread by malicious users, like human accounts or social bots, examining the profiles of such users usually suggests the how reliable of engaged users are.

User-based features can be extracted from individual and group level. Individual-user features means features extracted from an user account, which can be a strong indicator of the user credibility. Examples of this type of features are demographics information, including whether this account is verified, the number of followers and followees and the registration time and location [76]. Group-user features aim to summarize the characteristics of a group of users [77]. True and false information usually disseminate in different communities, and it is assumed that the community features could reveal the veracity of information pieces. The most common way to capture group features is by aggregating individual features of involved users, such as taking the statistical average of followees or calculating the ratio of verified users $[48,78]$.

\subsubsection{Reply-based}

When engaging the propagation of misinformation, users often write replies to express their opinions. Such replies also indicate users' stance, such as supporting, denying or asking for further clarification. Features of a series of replies suggest reactions of the public and how these reactions change temporally. Hence researchers 
have studied the effectiveness of reply features, from three levels: individual, group, and temporal.

Individual-reply features are extracted from a single reply. As replies are actually texts, the previously mentioned text-based content features also apply in this case. Besides, stance has been recognized as an unique feature from user replies. Users expressed their stance either directly by clicking "thumbs up" and "thumbs down" in Facebook or implicitly by writing replies, from which researchers have identified stance as the unique reply feature. There are three types of coarse-grained stances: positive, negative and neutral. Some research considers the additional stance of unrelated that is more about topic similarity [20]. Positive and negative stance indicates an willingness and unwillingness to accept the posted claim to be true respectively.

Group-reply features are generated by aggregating features from relevant replies. Like group user features, this approach is a manifestation of "wisdom of crowds". Besides the way to compute the text-based features, we can, for example, count the respective number of positive, negative replies in the replies of a claim. A more comprehensive list of group-level reply features is summarized in [76].

Temporal-reply features consider how the reply features change along time [78]. It has been proposed to utilize unsupervised machine learning, such as an auto-encoder, to capture the temporal variation features [22,79]. For instance, how the stance of user replies vary has shown a correlation with claim veracity [80]. We can infer the claim veracity based on the temporal changes of stance values of all relevant posts.

As we analyse, reply-based models are quite similar to stance-based counterparts. This can be supported by the fact that stance-based models, by definition, utilize users' viewpoints from relevant post contents to infer the veracity of original claim. The differences come from more diverse operations of reply-based models: the group-reply and temporal-reply features are able to capture group activities and temporal evolution of stance. These extra information dimensions are important complements to individual-stance based models. 


\subsubsection{Network-based}

False information circulating in social media constitutes a network, where each node usually involves an engaged user and his/her reply. Such network is usually a subset of a user community where there is an echo chamber effect. The basic assumption is that the credibility of a claim is highly related to relevant social users and posts. This motivate researchers to capture the network characteristics in order to identify claim veracity. Although the network-based approach often involves user replies in nodes, which has overlap with the reply-based approach, the former focuses more on the network structure and thus is a hybrid of the content and network information

There are multiple types of ways to define networks. Stance network is built based on stance of user replies that are relevant to the claim being verified. Each node is a user reply and each edge is the stance value [81,82]. The stance network could be directional that indicates which user has a stance toward which claim. Friendship network is constructed based on the friendship relationship among engaged users. Each node is a user and each edge can be directed or undirected, depending on how the concept of friendship is defined by the social platform [48]. For example, the friendship in Twitter is by the following/followee structure that is directional while it is different in Facebook. Propagation network extends the friendship network by tracking the trajectory of the spread of a claim [48]. Both homogeneous and heterogeneous networks can be built for the propagation process. Homogeneous networks are defined as those involving a single type of entities, such as posts or events [81] while heterogeneous networks consist of different types of entities, such as posts and users [83,84]. Generally, propagation networks of false information are distinct from those of true information in terms of node features, network width and the speed of temporal evolution.

After constructing these networks, various network metrics have been tried to extract features. For instance, researchers have proposed to extract the commonly used degree and clustering coefficient of the friendship network and propagation network [48]. Others use singular value decomposition [22] or propagation algo- 
rithms [81] to obtain latent embeddings of nodes. Recent misinformation analysis models use static graph neural networks to extract geometric propagation patterns; others leverage time-series analysis by treating misinformation spread as a temporal event sequence $[22,23]$. These two approaches each neglect the alternative propagation structure with neither leveraging both geometric and temporal dissemination features. Effective methods to exploit network interactions are an open area of research and need further investigations.

\subsubsection{Recap}

This subsection analyses the approaches to identifying false information. Content-based approaches alone are capable to achieve acceptable performance. Knowledge-based approaches are deficient for newly-emerging, time-critical events. Social context-based approaches utilize reactions of the public on social media that provides rich auxiliary cues in users, replies and networks. When developing network-based algorithms, researchers usually incorporate user account features and reply content into network nodes. Due to the existence of reply, the network-based approach is more like a hybrid machine learning model with features coming from both the network structure and the claim/reply content in nodes.

\subsection{Complementary Research Areas}

Due the complexity of the veracity evaluation, the research problem can be addressed from different perspectives. We point out the links and differences between automated veracity evaluation and these listed areas by summarily describing the task goals and reviewing typical algorithms.

\subsubsection{Check-Worthiness Prediction}

The task of check-worthiness prediction is to decide whether a piece of text includes a claim that is of interest to public audience and potentially exert negative influence. Check-worthiness can be predicted in political debates and social posts. Checkworthy claims can be defined as those convey key opinions or statements [19]. It is viewed as the first step in the fact-checking pipeline, before claim verification with various methods mentioned in subsection 2.3. 


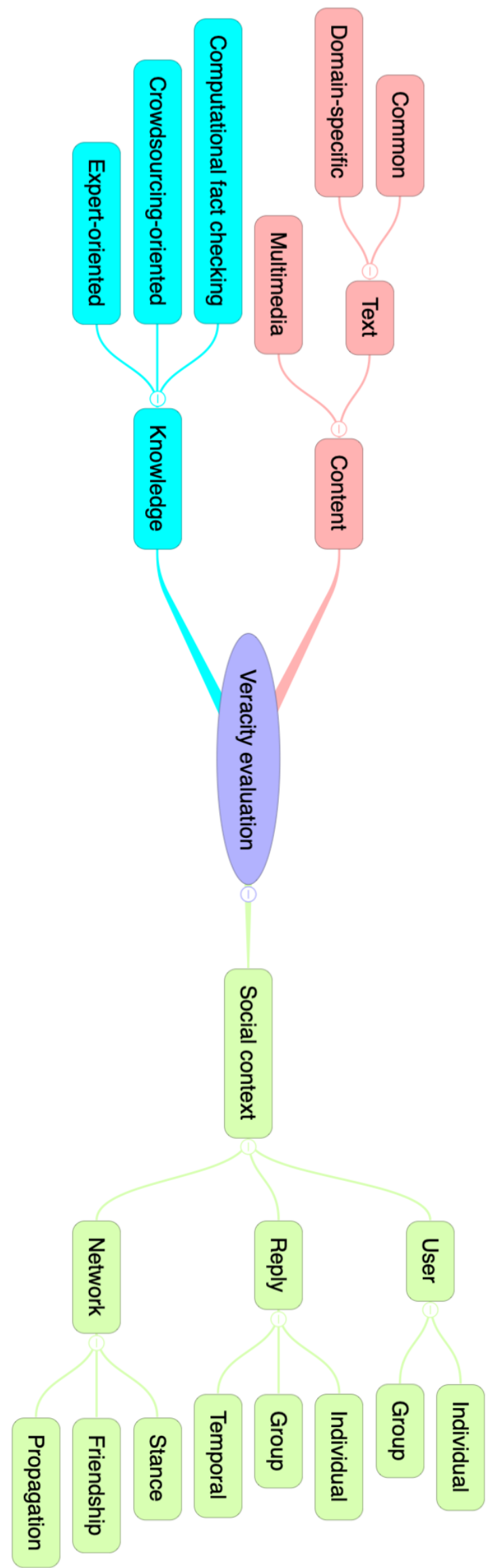

Figure 2.3: Mind map of the features and models for veracity evaluation. 
There have been organized challenges named CheckThat! that focus on checkworthiness prediction ${ }^{11}$. A public benchmark dataset, called ClaimBuster ${ }^{12}$ is developed $[85,86]$ to accelerate studies in this area. Researchers mainly rely on lexical and syntactic features extracted from the piece of text [87], such as Part-ofSpeech tags, named entities and Syntactic Dependency Trees. Contextual features have mainly been from pre-trained word/sentence embeddings based on neural networks, such as FastText [88] and Bert [52]. Statistical machine learning models, including Support Vector Machine (SVM) and Gradient Boosting Decision Tree, have achieved promising performance.

\subsubsection{Stance Detection}

Stance detection is defined as the task of identifying from a piece of text what stance is hold for a target entity [89]. Various texts are under study: social media posts [90] and news articles [91]. In the context of news stance detection, there are four stance classes: agree, disagree, discuss and unrelated [92]. Machine learning has been widely researched to tackle the task of stance detection. Most of previous works rely on content-based features, such as sentiment analysis and topic-specific features learned from labeled datasets.

This task has been studied in difference use cases: political debates, online products and misinformation detection. Besides as a task on its own right, stance detection is an important step towards detecting online false information in a number of ways. Supportive stance of an evidential article [92,93] from reputed sources indicates a true claim. Researchers have also studied the difference of true and false information in how user stance changes along time in social media [94]. Tacchini et al. [82] constructed a bipartite network of online users and social posts using agree stances, and developed a semi-supervised probabilistic algorithm to distinguish hoax and non-hoax posts. They further explored to utilize these stances in order to reveal the credibility of claims being verified. More details of how stance detection helps misinformation detection can be found in the survey [95].

\footnotetext{
11https://sites.google.com/view/clef2020-checkthat/

12 https: / / zenodo.org/record/3609356\#.Xywq-BP 0mL4
} 
Stance detection has also been studied in the context of social media. The public often express their stance towards certain targets through comments and replies on social platforms like Twitter and Facebook. The SemEval-2016 Task 6 formulates the stance detection task in Tweeter as follows: "given a tweet text and a target entity (person, organization, movement, policy, etc.), automatic natural language systems must determine whether the tweeter is in favor of the given target, against the given target, or whether neither inference is likely" [96]. The targets can be a person ("Hillary Clinton" and "Donald Trump"), a movement ("Feminist Movement"), a policy ("Legalization of Abortion") and an organization ("Atheism" and "Climate Change is a Real Concern"). The stance classes of user replies in the SemEval-2017 Task 8 and Pheme datasets are defined to be supporting, denying, commenting and questions [97]. The top ranked systems extracted common text features such as those n-grams, word vectors, and sentiment lexicons. These features are quite useful in the task of stance detection regardless of the context of social media or news articles. We use some of these features to constitute the input to our hierarchical stance detection model in Chapter 4. One concern is that the task setting does not involve unrelated that appears in news stance detection, so we have to find features for relevancy classification in Chapter 4.

We chose to study the stance detection task in the context of online news, because it simplifies veracity evaluation on the premise that supportive stances from reputed articles can prove claim truthfulness. Other contexts, such as social media conversations, usually involves users' instant reactions without careful investigations. Hence stance in these contexts alone cannot be reliably used to identify falsehood, but usually acts as an auxiliary signal in a multi-task setting, as we will discuss in the rumour classification.

\subsubsection{Rumor Classification}

A rumor is defined as "a piece of circulating information whose veracity status is yet to be verified at the time of spreading" [98]. Various studies for rumor analysis can be divided into four subtasks: rumor detection, rumor tracking, rumor stance classification, and veracity classification [98], which together make a pipeline. The 
first subtask, i.e., rumor detection, aims to identify whether a piece of information is rumor or not $[99,100]$; the second subtask, i.e., rumor tracking, attempts to collect and filter social media posts that are relevant to specific rumors; the third subtask, i.e., stance classification, aims to determine whether relevant posts confirms the rumor's veracity or not; last but not least, the forth subtask, i.e., veracity classification, predicts the veracity of the rumor, which is most related to this thesis. To decide rumor veracity, researchers often make use of the other subtasks, such as extracting stances or opinions from relevant posts. Stance detection has been studied in the context of not only online articles but also user replies in social media, to evaluate claim veracity. Motivated by the task setting of the SemEval-2017 Task 8, Kochkina et al. [97] show that the tasks of stance detection in user replies and rumour verification can be solved by multi-task learning with improved performance.

Rumor classification is quite relevant to our reply-based veracity evaluation in Chapter 5. Both of them rely on user comments and replies on social platforms to mine evidential information to indicate claim veracity. The major difference is that, as the above-mentioned, rumor classification is a sub-task of the broader rumor analysis, and thus has more interactions with the other three sub-tasks. For example, rumor analysis involves an extra unverified veracity class in its first sub-task while Chapter 5 focuses more on the classification of true and false. Rumor tracking is analogue to the engagement-based misinformation detection that we develop in Chapter 7 in terms of collecting temporally sequentially available user engagements.

\subsubsection{Truth Discovery}

The task of truth discovery aims to identify correct facts from multiple contradicting sources [101]. As a side product, this task determines the credibility of value sources. Instead of straightforwardly exploring fact statements, it depends on a set of conflicting sources that report attributes of objects to determine the correct value.

The research problem of veracity evaluation could benefit from the truth discovery technique. As mentioned, the credibility of conflicting sources can be inferred by truth discovery $[102,103]$. However, there are some concerns that must be 
considered before applying truth discovery techniques to automated veracity evaluation. First, truth discovery techniques usually take structured tuples as inputs, which are often the form of Subject-Predicate-Object; while this thesis have to deal with unstructured social posts. Second, truth discovery techniques require adequate number of sources, thus may not be feasible to tackle misinformation detection at early stages, during which time there are limited number of information outlets. Hence, we cannot directly utilize existing truth discovery techniques in this thesis.

\subsubsection{Source Credibility Analysis}

Source credibility analysis is a contributory research direction. As misinformation is usually published by untrustworthy individuals or automatic bots, credibility plays a crucial role in message communication [104,105]. Accurate and timely discrimination of such accounts inhibits the proliferation of misinformation at an early stage. [106] identify two components of source credibility, namely trustworthiness and expertise. Trustworthiness is generally taken to mean truthful, unbiased and well intentioned. Expertise instead is understood as knowledgeable, experienced and competent. Thus, features that can reveal the trustworthiness and expertise of information sources are strong indicators of source credibility. With the aid of information source [107] examine the credibility of tweets related to the Fukushima Daiichi nuclear disaster in Japan. They found that tweets from highly credible institutions and individuals are mostly correct. Useful account features can be derived from the account demographics, such as integrity of personal information, the number of followers and followees [76]. Besides, aggregating a group of account features are indicative, since spreaders of true and false claims might come from different communities [77], such as the percentage of verified user accounts [78] and the average number of followers [48]. Because of the importance of engaged user profiles, we extract user account features and incorporate them to node representations when we build the engagement graph in Chapter 7.

In addition, the rise of social bots has also increased the circulation of false information as they automatically retweet posts without verifying the facts [108]. The major challenge brought by social bots is that they can give a false impression 


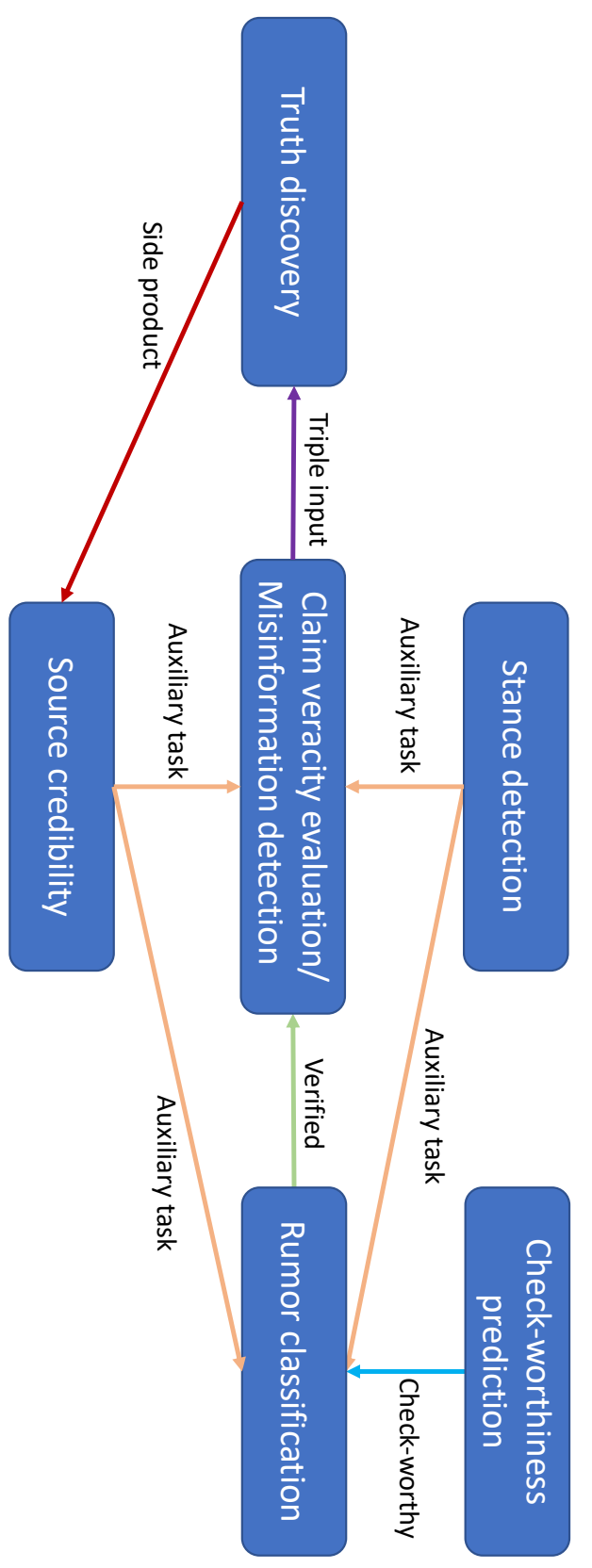

Figure 2.4: A diagram that shows how the listed research areas link to each other.

that information is highly popular and endorsed by many people, which enables the echo chamber effect for the propagation of misinformation. Previous approaches for bot detection are based on social network information, crowdsourcing, and discriminative features [108-110].

To visualize the relationships, Figure 2.4 shows how the listed research areas and tasks connect to each other. Each block represents an area and an arrow indicates how the starting area could be linked or converted to the ending area. For 
example, stance detection is seen as an auxiliary task for rumor classification, which is proved by improved performance of both by one multi-task model [97]. Besides, claim veracity evaluation in this thesis involves either true or false classes, which can be seen as a verified sub-class of rumour classification.

\subsection{Related Datasets}

Online news can be collected from different sources, such as news agency homepages, search engines, and social media websites. However, manually determining veracity is a challenging task, usually requiring annotators with domain expertise who performs careful analysis of claims and additional evidence, context, and reports from authoritative sources. Generally, news data with annotations can be gathered in the following ways: expert journalists, fact-checking websites, industry detectors, and crowdsourced workers. However, there is no agreement upon benchmark datasets for the veracity evaluation problem. Some publicly available datasets are listed below:

- FakeNewsChallenge ${ }^{13}$ : Ferreira and Vlachos [111] released a dataset for rumor debunking using data collected from the Emergent project [112]. For 300 claims, 2,595 corresponding news articles were collected and their stances were labeled as for, against or observing a claim. This dataset was extended for the 2017 false information Challenge dataset which consisted of approximately $50 \mathrm{~K}$ headline and body pairs derived from the original 300 claims and 2,595 articles.

- PHEME 14: The dataset includes rumor tweets, collected and annotated within the journalism use case of the project. These rumors are associated with 9 different breaking news. It contains Twitter conversations which are initiated by a rumorous tweet; the conversations include tweets responding to those rumorous tweets. These tweets have been annotated for support, certainty, and evidentiality.

\footnotetext{
${ }^{13}$ http://fakenewschallenge.org/

${ }^{14}$ https://figshare.com/articles/PHEME_rumour_scheme_dataset_ journalism_use_case/2068650
} 
- Twitter15 and Twitter16: Ma et al. [113] created two rumor datasets with tweets crawled from Twitter. There are 742 and 412 claims being verified in Twitter15 and Twitter 16 respectively. Claim veracity is manually annotated by fact-checkers. These dataset also provides rich social context on top of the original statements: (1) users that are involved in the propagation of statements, and (2) social engagements in the temporal order. There are about $190 \mathrm{~K}$ and $115 \mathrm{~K}$ social users involved in these two datasets respectively. Both datasets have over 200 retweets per claim on average.

We will give more details of the statistics of each dataset in the experiments of the following technical chapters.

\subsection{Summary}

Table 2.1 and 2.2 summarize existing veracity evaluation approaches based on different platforms and features. Efficient features are able to individually or jointly distinguish between true and false claims in various aspects. The most common features in false information detection are textual features, user account features and propagation features. Researchers derive such features from the characteristics analysis and show the differences in properties of true and false claims. These differences are then quantified by intelligently designed features. Supervised machine learning takes such features as input, where the target variable or label is known and explicitly used during model training. 


\begin{tabular}{l|l}
\hline Social Platform & Research Papers \\
\hline Twitter & $\begin{array}{l}\text { Bessi et al. [114], Ferrara et al. [108], Gupta et al. [2], } \\
\text { Howard et al. [115], Jin et al. [81, 116], Kim et al. [117], } \\
\text { Mendoza et al. [118], Mitra et al. [119, 120], Nied et al. [121], } \\
\text { Qazvinian et al. [122], Ruchansky et al. [22], Shah et al. [123], } \\
\text { Shao et al. [124, 125], Starbird et al. [126, 127], } \\
\text { Tripathy et al. [128], Vosoughi et al. [11], Zeng et al. [129], } \\
\text { Subrahmanian et al. [130], Zubiaga et al. [131] }\end{array}$ \\
Facebook & $\begin{array}{l}\text { Beutel et al. [132], Del et al. [38], Friggeri et al. [12], } \\
\text { Nguyen et al. [133],Silverman et al. [112], Tacchini et al. [82] }\end{array}$ \\
Sina Weibo & $\begin{array}{l}\text { Jiang et al. [134], Kim et al. [135], Ruchansky et al. [22], } \\
\text { Wu et al. [136], Yang et al. [77] }\end{array}$ \\
Cross-platforms & $\begin{array}{l}\text { Reddit+Twitter+4chan: Zannettou et al. [137] } \\
\text { other }\end{array}$ \\
& $\begin{array}{l}\text { False information articles: Horne et al. [138], Rubin et al. [139], } \\
\text { Wikipedia: Kumar et al. [1], } \\
\text { False information websites: Albright et al. [140, 141], } \\
\text { Fact checking website: Shu et al. [142] and Wang et al. [143], } \\
\text { Crowdsourcing and tabloid websites: Perez et al. [30] }\end{array}$ \\
\hline
\end{tabular}

Table 2.1: This table categorizes research on false information based on the platforms they study. 


\begin{tabular}{|c|c|}
\hline Feature Category & Research Papers \\
\hline Text & $\begin{array}{l}\text { Gupta et al. [2],Horne et al. [138], Howard et al. [115], } \\
\text { Kumar et al. [1],Mitra et al. [119, 120],Zeng et al. [129], } \\
\text { Yang et al. [77], Perez et al. [30], Qazvinian et al. [122], } \\
\text { Wang et al. [143],Rubin et al. [139], Silverman et al. [112], } \\
\text { Zubiaga et al. [131], }\end{array}$ \\
\hline User & $\begin{array}{l}\text { Yang et al. [77], Bessi et al. [114], Davis et al. [144] } \\
\text { Tacchini et al. [82], Gupta et al. [2], Jin et al. [81, 116], } \\
\text { Vosoughi et al. [11],Kumar et al. [1], Mendoza et al. [118], } \\
\text { Nied et al. [121],Shao et al. [124, 125], }\end{array}$ \\
\hline Graph & $\begin{array}{l}\text { Subrahmanian et al. [130], Bessi et al. [114], Daviset al. [144], } \\
\text { Vosoughi et al. [11],Friggeri et al. [12],Kumar et al. [1], } \\
\text { Starbird et al. [126,127], Mendoza et al. [118],Nied et al. [121], } \\
\text { Qazvinian et al. [122], }\end{array}$ \\
\hline Time & $\begin{array}{l}\text { Del et al. [38],Davis et al. [144],Friggeri et al. [12], } \\
\text { Shao et al. [124, 125], Vosoughi et al. [11], } \\
\text { Zannettou et al. [137], Zeng et al. [129] }\end{array}$ \\
\hline Propagation & $\begin{array}{l}\text { Vosoughi et al. [11], Friggeri et al. [12],Jin et al. [81, 116], } \\
\text { Zannettou et al. [137], Shao et al. [124, 125], Zeng et al. [129], } \\
\text { Yang et al. [77],Silverman et al. [112] }\end{array}$ \\
\hline
\end{tabular}

Table 2.2: This table categorizes research based on the characteristics and features of false information analyzed. 



\section{Chapter 3}

\section{Background}

This thesis sets out to evaluate claim veracity based on various machine learning models and algorithms. In this chapter, we present an introduction to the used machine learning techniques and relevant mathematical knowledge, which is a preliminary preparation for the following technical chapters. We begin by the presence of deep learning models, including convolutional and recurrent neural networks, the attention mechanism and latent variable models, followed by temporal point processes. Then we introduce the evaluation metrics that are commonly-used to compare machine learning performance.

\subsection{Deep Learning}

Deep learning, usually implemented as deep neural networks, is one of the most attention-attractive machine learning techniques [5] since 2010. Due to the availability of a large amount of data and great computing power, deep learning is able to recognize complex data patterns based on its strong capability of function approximation. Representative examples of deep learning can be Multi-Layer Perceptron (MLP), Convolutional Neural Networks (CNNs), Recurrent Neural Networks (RNNs), Graph Neural Networks (GNNs), the attention mechanism and the latent variable model. These models have achieved extensive success in computer vision [145], natural language processing [52], speech recognition [146], information retrieval [147], networks and graphs [148], etc. In what follows, we discuss the main idea and mathematical foundation of the models used by this thesis. 


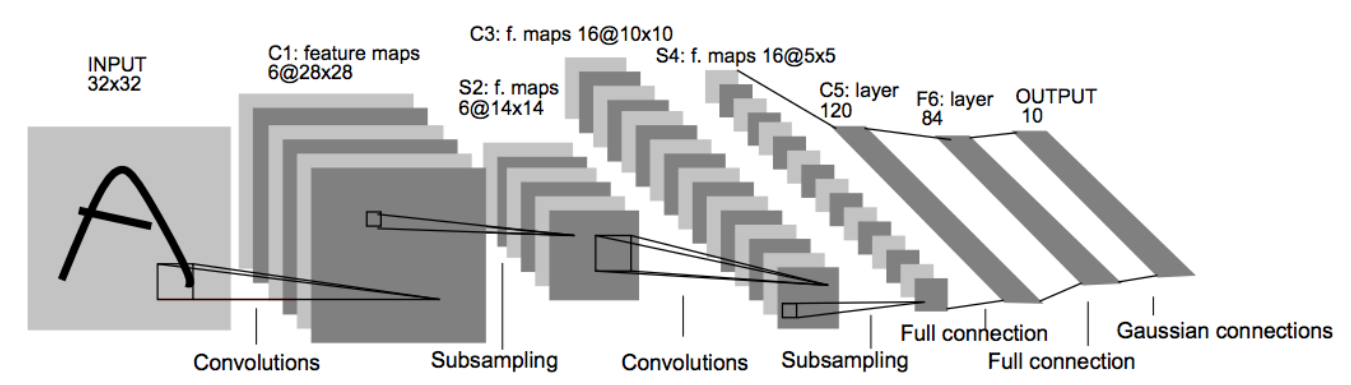

Figure 3.1: An example of the CNN model, LeNet-5, to classify characters. This figure is originally published in [4]

\subsubsection{Convolutional Neural Networks}

Convolutional neural network is a revolutionary architecture, which is initially proposed for computer vision and image understanding tasks [4] and then generalize to other tasks such as 1-dimensional sequence processing [149]. Similar to how human brains activate neurons in the visual cortex, CNNs learn to stimuli a limited area of the neuron units in its architecture, which is known as the receptive field. A set of such fields cover the whole visual region. Compared to conventional handengineered machine learning models, $\mathrm{CNNs}$ require simple data pre-processing and are able to learn proper feature extractors (a.k.a., filters) from training data. Figure 3.1 shows a typical CNN model for image classification.

There are a few essential modules in a typical CNN architecture: padding, striding, pooling and activation functions. We use padding to append certain values (usually zeros) to expand an image, based on which we compute the convolution covering all the input values. This ensures the size of the convolution output is the same as its input and we do not drop values at the input data boarder. Striding is employed to skip some neighbouring neuron units when it is not necessary to utilize all data in the input. Pooling aims to summarize a small portion of the input by taking an average of data in this portion (a.k.a., average pooling) or the maximum (a.k.a., maximum pooling). It can effectively reduce the size of its output. Activation functions are usually non-linear at the end of or between neural network modules. It decides the non-linearity of the whole neural network. There are different types of activation functions, with a representative being Rectified Linear Unit (ReLU). 


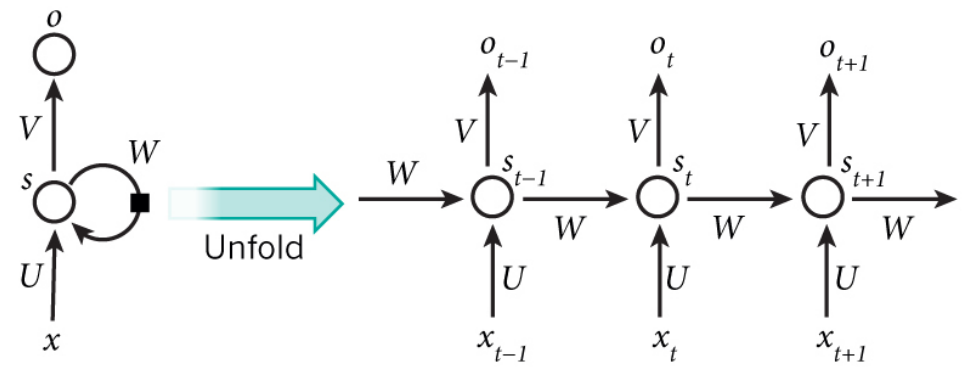

Figure 3.2: A recurrent neural network and the unfolding in time of the computation involved in its forward computation. This figure is originally published in [5].

\subsubsection{Recurrent Neural Networks}

Recurrent neural network is a type of neural networks that reuse previous outputs as inputs [5]. On top of feed-forward neural networks, RNNs can use the hidden states to memorize information from the input sequences with varying lengths. This characteristic allows RNNs to process sequentially organizing information such as natural language and speech. Take a sequence of words as an example. RNNs take one word as the input at a step and process them one by one as more words are sequentially available. The hidden state is supposed to contain information of the already seen data. At each forward step, the input word vector is concatenated to the hidden state and passes through an activation function to produce an output. Figure 3.2 unfolds a RNN in its forward computation.

RNNs are known to suffer from the problem of vanishing gradients when dealing with long sequences. Specifically, during the back-propagation phase, gradients from the beginning data of a sequence can be so small that RNN parameters cannot be efficiently updated to process these data. In order to address this problem, researchers developed two RNN variants: Long Short-Term Memory (LSTM) and Gated Recurrent Units (GRUs). Both models use internal gates in neuron units to control how information flows and determine which features should be kept for the task and which features can be thrown away. LSTM has three types of gates (input, output, and forget) to control information flow while GRU only uses two types of gates (update and reset). Because of fewer tensor calculations, GRU is reported to be faster to train while producing similar performance to LSTM [150]. 


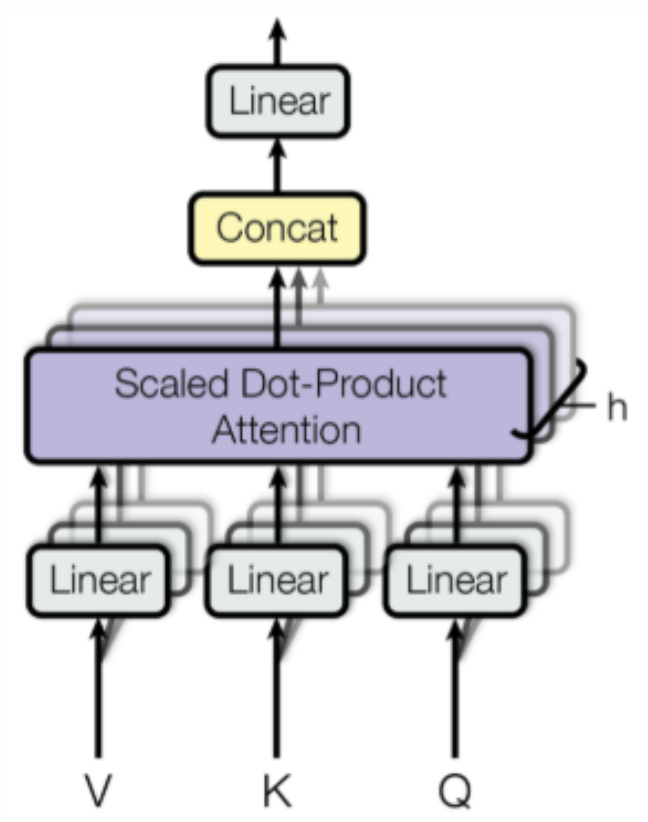

Figure 3.3: An illustration of self-attention. This figure is originally published in [6].

\subsubsection{Attention \& Self-Attention}

The attention mechanism enables to focus on a subset of the input sequence [151]. In sequence-to-sequence models with the attention, the input is represented with a sequence of key vectors $K$ and value vectors $V,(K, V)=\left[\left(k_{1}, v_{1}\right), \ldots,\left(k_{N}, v_{N}\right)\right]$. While, the output uses query vectors, $Q=\left[q_{1}, q_{2}, \ldots, q_{M}\right]$. These query vectors are used to find which part of the input sequence is more contributory [6].

Given these two sequences of vectors $(K, V)$ and $Q$, the attention mechanism computes a prediction sequence $O=\left[o_{1}, o_{2}, \ldots, o_{M}\right]$ as follows:

$$
o_{m}=\left(\sum_{n} f\left(q_{m}, k_{n}\right) g\left(v_{n}\right)\right) / \sum_{n} f\left(q_{m}, k_{n}\right),
$$

where $m \in\{1, \ldots, M\}, n \in\{1, \ldots, N\}, q_{m} \in \mathbb{R}^{d}, k_{n} \in \mathbb{R}^{d}, v_{n} \in \mathbb{R}^{p}, g\left(v_{n}\right) \in \mathbb{R}^{q}$ and $o_{m} \in$ $\mathbb{R}^{q}$. The similarity function $f\left(q_{m}, k_{n}\right)$ characterizes the relation between $q_{m}$ and $k_{n}$, whose common form is composed of: an embedded Gaussian, an inner-product, and a concatenation [152]. The function $g\left(v_{n}\right)$ is a linear transformation specified as $g\left(v_{n}\right):=v_{n} W_{v}$, where $W_{v} \in \mathbb{R}^{p \times q}$ is a weight matrix.

Self-attention is a special case of the attention mechanism [6], where the query vectors $Q$, like $(K, V)$, are from the encoder side. Self-attention is a method of 
encoding sequences of input tokens by relating these tokens to each other based on a pairwise similarity function $f(\cdot, \cdot)$. It measures the dependency between each pair of tokens from the same input sequence. To encode position information of tokens, position embeddings are calculated based on order numbers in a sequence. Consequently, self-attention encodes both token similarity and position information.

Self-attention is very expressive and flexible for both long-term and local dependencies, which used to be modeled by RNNs and CNNs [6]. Moreover, the self-attention mechanism has fewer parameters and faster convergence than RNNs. Recently, a variety of NLP tasks have experienced large improvements thanks to self-attention $[6,52]$.

\subsubsection{Latent Variable Model}

Probabilistic Latent Variable Model involves a random hidden variable with a distribution, which is different from the deterministic hidden variables in the abovementioned deep neural networks. The latent variable is supposed to capture the unseen factors from the input; its distribution indicates the uncertainty caused by these unseen factors [153]. One way to obtain the distribution of the latent variable is variational inference, based on which a Variational Auto-Encoder (VAE) is developed. The model training process is equivalent to maximizing a lower bound of data likelihood (referred to as the evidence lower bound, or ELBO).

$$
\begin{aligned}
\mathcal{L} & =\mathbb{E}_{\mathrm{q}_{\phi}(\mathrm{z} \mid \mathrm{x})} \log p_{\theta}(x \mid z)-K L\left[q_{\phi}(z \mid x) \| p_{\theta}(z)\right] \\
& =\sum_{i}\left[\mathbb{E}_{\mathrm{q}_{\phi}\left(\mathrm{z}_{\mathrm{i}} \mid \mathrm{x}_{\mathrm{i}}\right)} \log p_{\theta}\left(x_{i} \mid z_{i}\right)-K L\left[q_{\phi}\left(z_{i} \mid x_{i}\right) \| p_{\theta}\left(z_{i}\right)\right]\right]
\end{aligned}
$$

The basic intuition behind this objective is that the first term acts as a reconstruction loss and the Kullback-Leibler (KL) term acts as a regularizer. It usually sets a unit diagonal Gaussian prior on the latent variable: $p_{\theta}(z)=\mathcal{N}(0, I)$, and learns the distributions $q_{\phi}(z \mid x)$ and $p_{\theta}(x \mid z)$ jointly. As shown in Figure 3.4, the encoder network, also known as the inference network, maps data into a distribution over a latent space while the decoder network, also known as the generative network, maps samples from the latent space back into the original data space. 


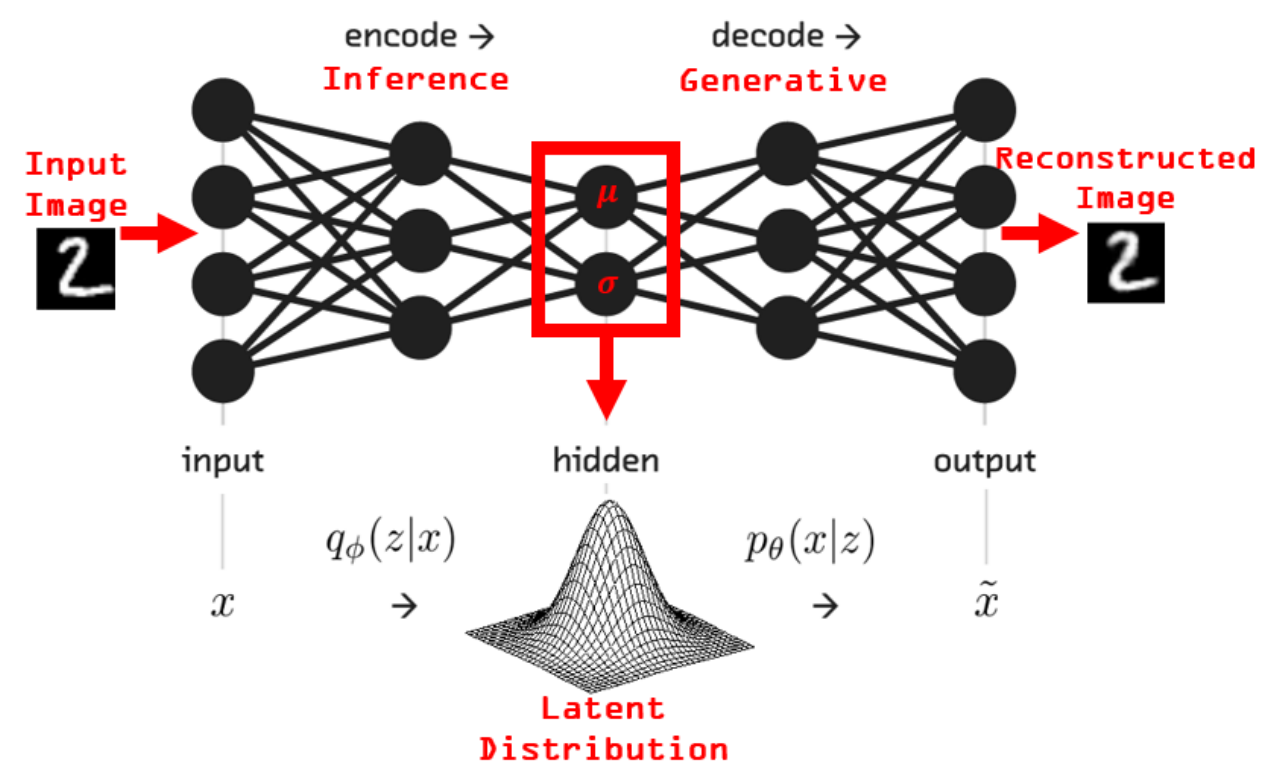

Figure 3.4: An illustration of VAE. This figure is originally published in [7].

\subsection{Temporal Point Processes}

A temporal point process is a stochastic process whose realization is a list of discrete events on the continuous time $t \in \mathbb{R}^{+}[154,155]$. A marked TPP allocates a type (a.k.a. mark) $u$ to each event. TPPs can be equivalently represented as a counting process $N(t)$, which records the number of events that have happened till time $t$. A multivariate TPP describes the temporal evolution of multiple event types $\mathcal{U}$.

We indicate with $\mathcal{S}=\left\{\left(v_{i}, t_{i}\right)\right\}_{i=1}^{L}$ an event sequence, where the tuple $\left(v_{i}, t_{i}\right)$ is the $i$-th event of the sequence $\mathcal{S}, v_{i} \in \mathcal{U}$ is the event type, and $t_{i}$ is the timestamp of the $i$-th event. We indicate with $\mathcal{H}_{t}:=\left\{\left(v^{\prime}, t^{\prime}\right) \mid t^{\prime}<t, v^{\prime} \in \mathcal{U}\right\}$ the historical sequence of events that happened before $t$. One typical way to characterize point processes is via an intensity function $\lambda^{*}(t)$, where $*$ means the intensity function is history dependent.

Given an infinitesimal time window $[t, t+\mathrm{d} t)$, the intensity function of a TPP is defined as the probability of the occurrence of an event $\left(v^{\prime}, t^{\prime}\right)$ in $[t, t+\mathrm{d} t)$ conditioned on the history of events $\mathcal{H}_{t}$ :

$$
\begin{aligned}
\lambda^{*}(t) \mathrm{d} t: & =P\left(\left(v^{\prime}, t^{\prime}\right): t^{\prime} \in[t, t+\mathrm{d} t) \mid \mathcal{H}_{t}\right) \\
& =\mathbf{E}\left(\mathrm{d} N(t) \mid \mathcal{H}_{t}\right)
\end{aligned}
$$




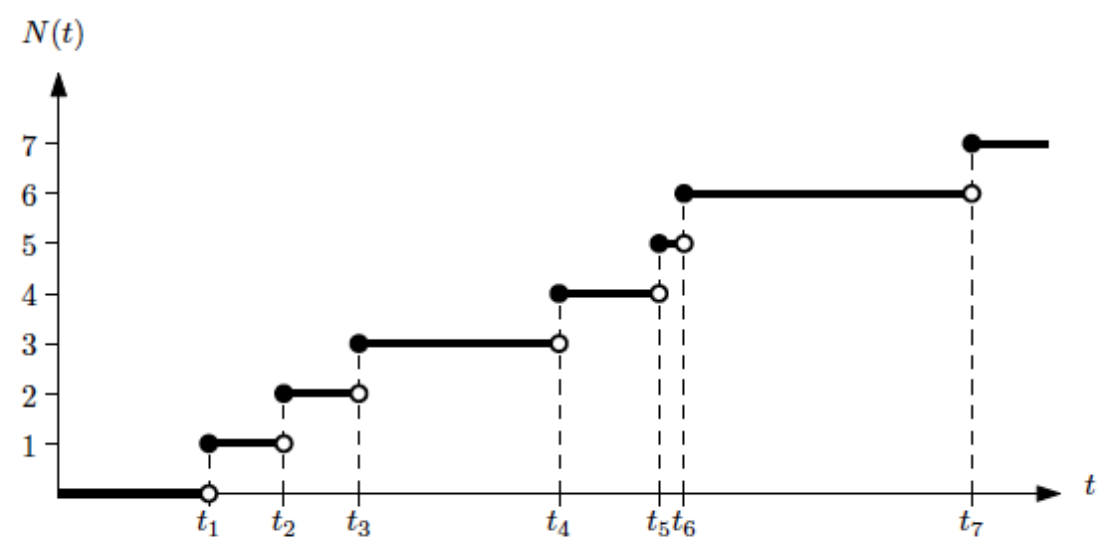

Figure 3.5: An example of the point process realization and it counting process. This figure is originally published in [8].

where $\mathbf{E}\left(\mathrm{d} N(t) \mid \mathcal{H}_{t}\right)$ denotes the expected number of events in $[t, t+\mathrm{d} t)$ based on the to the history $\mathcal{H}_{t}$. Without loss of generality, we assume that two events do not happen simultaneously, i.e., $\mathrm{d} N(t) \in\{0,1\}$.

Based on the intensity function, it is straightforward to derive the probability density function $p^{\star}(t)$ and the cumulative density function $F^{*}(t)$ [156]:

$$
\begin{gathered}
p^{*}(t)=\lambda^{*}(t) \exp \left(-\int_{t_{i-1}}^{t} \lambda^{*}(\tau) \mathrm{d} \tau\right) \\
F^{*}(t)=1-\exp \left(-\int_{t_{i-1}}^{t} \lambda^{*}(\tau) \mathrm{d} \tau\right) .
\end{gathered}
$$

\subsubsection{Poisson process}

A Poisson process is a type of temporal point process, where the intensity is history independent [157]. There are two types of Poisson processes: homogeneous and inhomogeneous. A homogeneous Poisson process has a constant intensity $\lambda^{*}(t):=$ $\mu \geq 0$, while a inhomogeneous Poisson process is defined by a time varying function $\lambda^{*}(t):=\psi(t) \geq 0$.

\subsubsection{Hawkes process}

An Hawkes process [158] models the self-excitation of events of the same type and the mutual excitation of different event types, in an additive way. Hence, the 


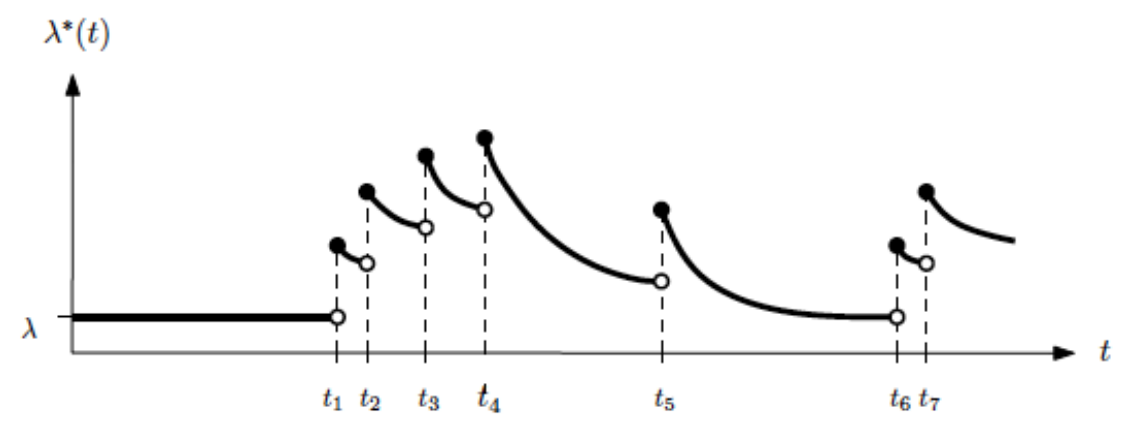

Figure 3.6: An example of the intensity function of an Hawkes process. This figure is originally published in [8].

definition of the intensity function is given as:

$$
\lambda^{*}(t)=\mu+\sum_{\left(v^{\prime}, t^{\prime}\right) \in \mathcal{H}_{t}} \phi\left(t-t^{\prime}\right)
$$

where $\mu \geq 0$ (a.k.a. base intensity) is an exogenous component of the intensity function independent of the history, while $\phi(t)>0$ is an endogenous component that is dependent on the history. Besides, $\phi(t)$ is a triggering kernel that contains the peer influence of different pairs of event types. To highlight the peer influence represented by $\phi(t)$, we write $\phi_{u, v}(t)$, which captures the impact of a historical type- $v$ event on a subsequent type- $u$ event [159]. In the example of Equation 3.6, the occurrence of a past type- $v$ event increases the intensity function by $\phi_{u, v}\left(t-t^{\prime}\right)$ for $0<t^{\prime}<t$.

Most commonly $\phi_{u, v}(t)$ is parameterized as $\phi_{u, v}(t)=\alpha_{u, v} \cdot \kappa(t) \cdot \mathbb{1}_{t>0}[160,161]$. The excitation parameter $\alpha_{u, v}$ quantifies the initial influence of the type- $v$ event on the intensity of the type- $u$ event. The kick function $\kappa(t)$ characterizes the time-decaying influence. Typically, $\kappa(t)$ is chosen to be exponential, i.e., $\kappa(t)=$ $\exp (-\gamma t)$, where $\gamma$ is the decaying parameter controlling the decaying speed.

\subsubsection{Self-correcting process}

A self-correcting process assumes the occurrence of history events inhibits the occurrence of future events [162]. The intensity function is defined as $\lambda *(t)=$ $\exp \left(\mu t-\sum_{\left(v_{i}, t_{i}\right) \in \mathcal{H}_{t}} \alpha\right)$. 


\subsubsection{Optimization}

To learn the parameters of temporal point processes, it is common to use Maximum Likelihood Estimation (MLE). Other advanced and more complex adversarial learning [163] and reinforcement learning [164] methods have been proposed. To apply MLE, a loss function is derived based on the negative log-likelihood.

Given the history $\mathcal{H}_{t}$ and the next timestamp $t_{i}$, the probability of $v_{i}$ is

$$
p\left(u=v_{i} \mid t_{i}\right)=\frac{\lambda_{v_{i}}\left(t_{i}\right)}{\lambda\left(t_{i}\right)},
$$

where $\lambda(t)=\sum_{u=1}^{u=|\mathcal{U}|} \lambda_{u}(t)$. The log-likelihood of an event sequence $\mathcal{S}$ over a time interval $[0, T]$ is given by:

$$
\begin{aligned}
\mathcal{L} & =\log \left[\prod_{i=1}^{L} p\left(t_{i}\right) p\left(u=v_{i} \mid t_{i}\right)\right] \\
& =\log \left[\prod_{i=1}^{L} \lambda\left(t_{i}\right) \exp \left(-\int_{t_{i-1}}^{t_{i}} \lambda(\tau) \mathrm{d} \tau\right) \frac{\lambda_{v_{i}}\left(t_{i}\right)}{\lambda\left(t_{i}\right)}\right] \\
& =\log \left[\exp \left(-\sum_{i=1}^{L} \int_{t_{i-1}}^{t_{i}} \lambda(\tau) \mathrm{d} \tau\right) \prod_{i=1}^{L} \lambda_{v_{i}}\left(t_{i}\right)\right] \\
& =\log \left[\exp \left(-\int_{0}^{T} \lambda(\tau) \mathrm{d} \tau\right) \prod_{i=1}^{L} \lambda_{v_{i}}\left(t_{i}\right)\right] \\
& =\sum_{i=1}^{L} \log \lambda_{v_{i}}\left(t_{i}\right)-\int_{0}^{T} \lambda(\tau) d \tau
\end{aligned}
$$

where the first term is the sum of the log-intensity functions of past events, and the second term corresponds to the log-likelihood of infinitely many non-events. By non-event, we mean there are no events at a certain timestamp. Intuitively, the probability that there is no event of any type in the infinitesimally time interval $[t, t+d t)$ is equal to $1-\lambda(t) d t$, the $\log$ of which is $-\lambda(t) d t$.

To maximize the log-likelihood objective with stochastic gradient methods, we need to get an unbiased estimate of the gradient with respect to the model parameters. Monte Carlo sampling is used to estimate the gradients of the integral part in Equation 3.8. It takes the average of several samples and thus reduces the variance of gradient estimation, which is also used in [165]. 


\subsection{Evaluation}

In this section, we discuss how to evaluate the performance of machine learning algorithms for veracity evaluation. As we treat veracity evaluation as a binary classification problem, we focus on the classification evaluation metrics. Classification algorithms are usually evaluated based on the following metrics: Accuracy, $\mathrm{F}_{1}$, Precision, and Recall. Accuracy is defined as the fraction of correct predictions over all predictions:

$$
\text { Accuracy }=\frac{T P+T N}{T P+T N+F P+F N}
$$

where $T P=$ True Positives (correctly predicted true claims), $T N=$ True Negatives (correctly predicted false claims), $F P=$ False Positives (false claims predicted as true), and $F N=$ False Negatives (true claims predicted as false). Precision is defined as the fraction of true claims predicted as true over all predicted claims:

$$
\text { Precision }=\frac{T P}{T P+F P} \text {. }
$$

and Recall is defined as the fraction of true claims predicted as true over all true claims:

$$
\text { Recall }=\frac{T P}{T P+F N}
$$

$F_{1}$ is defined as the harmonic mean of Precision and Recall:

$$
F_{1}=2 \cdot \frac{\text { Precision } \cdot \text { Recall }}{\text { Precision }+ \text { Recall }}
$$

Although Accuracy is commonly used for classification tasks, it is less reliable when datasets suffer from class imbalance. The evaluation measures Precision, Recall and $\mathrm{F}_{1}$ complement Accuracy, and $\mathrm{F}_{1}$ is selected as a single metric that indicates the overall performance when datasets have a class imbalance problem.

The above evaluation metrics are commonly used to compare machine learning algorithms. They allows us to evaluate the performance of a classifier from different perspectives. Specifically, Accuracy measures the similarity between predicted misinformation and real misinformation. Precision measures the fraction of all de- 
tected misinformation that are annotated as misinformation, addressing the important problem of identifying which news is fake. However, because misinformation datasets are often skewed, a high Precision can be easily achieved by making fewer positive predictions. Thus, Recall is used to measure the sensitivity, or the fraction of annotated misinformation articles that are predicted to be misinformation. $F_{1}$ is used to combine Precision and Recall, which can provide an overall prediction performance for veracity evaluation. Note that for all the metrics, Precision, Recall, $F_{1}$ and Accuracy, the higher the values are, the better performance one model achieves.

\subsection{Summary}

This section introduces preliminary concepts of deep neural networks, temporal point processes, and the evaluation metrics that we use in this thesis. It paves way for the following technical chapters. 



\section{Chapter 4}

\section{Hierarchical Stance Representation and Detection for Online News}

In this chapter, we present our research work regards the stance detection task for online news - a hierarchical model for stance representation and detection. This work has been accepted as a full paper by the Web Conference 2019 [166].

\subsection{Description of Stance Detection Task}

Stance detection plays a significant role in veracity evaluation, as discussed in Literature Review. It can contribute from following aspects: (1) supporting/opposing stances from credible sources can be directly used as powerful evidence to determine veracity; (2) false information often triggers versatile, mostly controversial stances, thus joint detection of stance and veracity helps extract prominent, invariant features; (3) stances can also be used to infer credibility of unknown sources, based on the assumption that credible sources are more likely to support true information and vice versa.

The task we address is to detect the stance of a piece of text towards a claim as one of the four classes: agree, disagree, discuss and unrelated [20].

- agree - the piece of text supports the claim.

- disagree - the piece of text denies the claim.

- discuss - the text discusses the same topic as the claim, but does not take a position.

- unrelated - the text discusses a different topic but not that in the claim. 


\subsection{Related Works}

Machine learning techniques are widely researched to tackle the stance detection task. We briefly analyse the previous stance detection works in the context of online news articles. Most of these works rely on content-based features, such as sentiment analysis and topic-specific features learned from labeled datasets for a closed set of topics, and performed well in the Fake News Challenge in $2017^{1}$.

Some methods use separate models to classify the four types of stances. The lemmatized $n$-gram matching and a rule-based procedure is used to decide the text relatedness, and a three-way logistic regression classifier to distinguish among the relevant classes [167]; a gradient boosted decision tree (GBDT) model [168] is developed to determine the text relatedness, then another GBDT model is used to distinguish stances of the text towards the claim [169]. These methods involve feature engineering in separate models and cannot be jointly optimized to achieve the best performance.

The first stage of Fake News Challenge (FNC-1) [170] is actually a stance detection task. The winner team uses a 50\%/50\% weighted average between a GBDT model and a convolutional neural network (CNN) [171]. The second best performance is achieved by an ensemble of five multi-layer perceptrons (MLPs) where input features include bag-of-words, semantic analysis in addition to the baseline features developed by the challenge organizers [172]. Compared to the above two solutions, the third best team does not try ensemble methods. They use TF-IDF features and an MLP as a four-way classifier [91]. A ranking method is proposed to tackle the task and achieve empirical performance improvements [20]. However, these methods all neglect the hierarchical structure among the four types of stances and suffer from class imbalance.

Deep learning-based methods have also been applied. Bajajpope et al. [173] utilize LSTM, CNN and their variants to detect stances. They find that an attentionaugmented CNN obtains the best performance. The effectiveness of different text coding, such as independent coding, bidirectional conditional encoding and atten-

\footnotetext{
${ }^{1}$ http://www. fakenewschallenge.org/
} 


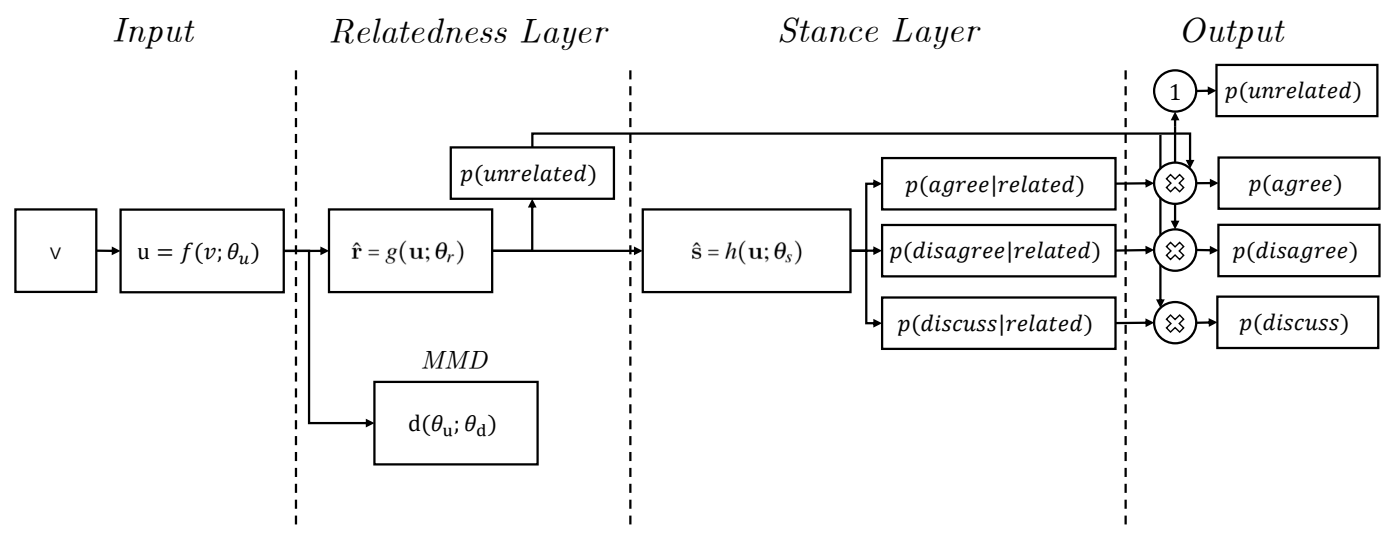

Figure 4.1: The architecture of our proposed two-layer neural network.

tive readers is analysed in [174]. A multi-task learning algorithm is proposed to jointly detect rumours and stances [113]. However, all these methods fail to achieve high accuracy for the agree and disagree classes.

There are three major defects in all the aforementioned methods: (a) they neglect the hierarchical relationships among the four stances; (b) they suffer from the class imbalance problem, and; (c) they fail to achieve acceptable detection performance for the agree and disagree classes.

\subsection{Hierarchical Classification}

In this section, we detail our proposed two-layer neural network for stance detection. $\S 4.3 .1$ outlines the model. In order to better differentiate between the related and unrelated classes, we design an MMD regularization term in $\S 4.3 .2$. This is then integrated into the two-layer neural network loss function in $\S$ 4.3.3. In Figure 4.1, we show the architecture of our model.

\subsubsection{Two-Layer Neural Network}

Let the input space be formed by $m$-dimensional real vectors in a neural network, denoted as $\mathbf{v} \in \mathbb{R}^{m}$. The four-class label can be transformed into a one-hot vector $\mathbf{y}$. The $i$-dimension of $\mathbf{y}\left(y_{i}\right)$ is 1 when the stance is the $i$-element in the label set $\{$ agree, disagree, discuss, unrelated $\}$ and 0 otherwise. The hidden layer with 
74 Chapter 4. Hierarchical Stance Representation and Detection for Online News

parameters $\theta_{u}$ learns to map $\mathbf{v}$ to a $k$-dimensional hidden representation $\mathbf{u} \in \mathbb{R}^{k}$ :

$$
\mathbf{u}=f\left(\mathbf{v} ; \theta_{u}\right)
$$

For the two-layer classification, the first layer decides whether the text is related to a claim. Hence, the first classification layer is called the relatedness layer. This layer is parameterized by $\theta_{r}$ and learns to produce a 2 -dimensional normalized vector $\hat{\mathbf{r}}$ as follows:

$$
\hat{\mathbf{r}}=g\left(\mathbf{u} ; \theta_{r}\right)
$$

Note that the Softmax function is included in $g$ to normalize the 2-dimensional vector, so each component of the vector $\hat{\mathbf{r}}$ denotes the probability that the neural network assigns $\mathbf{v}$ to the related and unrelated classes, i.e., p(related) and p(unrelated $)$.

The second layer classifies the text into the related classes, i.e., agree, disagree, or discuss stances. Hence, the second classification layer is called the stance layer. The stance layer is parameterized by $\theta_{s}$ and learns to produce a 3-dimensional normalized vector $\hat{\mathbf{s}}$ :

$$
\hat{\mathbf{s}}=h\left(\mathbf{u} ; \theta_{s}\right)
$$

Note that the Softmax function is also included in $h$ to normalize the 3-dimensional vector, so that each component of the vector $\hat{\mathbf{s}}$ denotes the conditional probability that the neural network assigns $\mathbf{v}$ to agree, disagree and discuss given that $\mathbf{v}$ is related, i.e., $p$ (agree|related $), p($ disagree $\mid$ related $)$, and $p($ discuss $\mid$ related $)$.

We define the classification loss by the Kullback-Leibler (KL) divergence [175], which measures the difference between the network outputs and labels:

$$
l^{r}\left(\theta_{u}, \theta_{r}\right):=\operatorname{KL}(\mathbf{r} \| \hat{\mathbf{r}})
$$

where $\mathbf{r}$ is the ground-truth relatedness of the input data. $\mathbf{r}$ is computed from a label $\mathbf{y}$ as follows:

$$
\mathbf{r}=\left(\mathbb{1}\left(\mathbf{y} \neq \mathbf{e}_{4}\right), \mathbb{1}\left(\mathbf{y}=\mathbf{e}_{4}\right)\right),
$$


where $\mathbb{1}$ is the indicator function, $\mathbf{e}_{4}$ is a 4-dimensional one-hot vector with fourth element equal to 1 . When $\mathbf{y}=\mathbf{e}_{4}$ is verified, it indicates that the label belongs to the unrelated class. Similarly, the stance classification loss can be defined as:

$$
l^{s}\left(\theta_{u}, \theta_{r}, \theta_{s}\right):=\operatorname{KL}(\mathbf{s} \| \hat{\mathbf{s}}),
$$

where $\mathbf{s}$ is the ground-truth stance of the input data. $\mathbf{s}$ is computed from a label $\mathbf{y}$ as follows:

$$
\mathbf{s}=\left(\mathbb{1}\left(\mathbf{y}=\mathbf{e}_{1}\right), \mathbb{1}\left(\mathbf{y}=\mathbf{e}_{2}\right), \mathbb{1}\left(\mathbf{y}=\mathbf{e}_{3}\right)\right)
$$

where $\mathbf{e}_{1}, \mathbf{e}_{2}, \mathbf{e}_{3}$ are 4-dimensional one-hot vectors with first, second, and third elements equal to 1 . When $\mathbf{y}=\mathbf{e}_{1}$ is verified, it indicates that the label belongs to the agree class, when $\mathbf{y}=\mathbf{e}_{2}\left(\right.$ or $\left.\mathbf{y}=\mathbf{e}_{3}\right)$ is verified, it indicates that the label belongs to the disagree (or discuss) class.

Finally, we now define the loss function for the two-layer neural network as the linear combination between the loss function of the relatedness layer $\left(l^{r}\right)$ and the loss function of the stance layer $\left(l^{s}\right)$ :

$$
l^{c}\left(\theta_{u}, \theta_{r}, \theta_{s}\right):=l^{r}\left(\theta_{u}, \theta_{r}\right)+\alpha \cdot l^{s}\left(\theta_{u}, \theta_{r}, \theta_{s}\right)
$$

where $\alpha$ leverages the importance of the two classification layers.

\subsubsection{Maximum Mean Discrepancy}

As analysed before, the classification of related/unrelated stances is a different task from that of agree/disagree/discuss stances. We classify relatedness in the first layer and the fine-grained stance in the second layer. Data representations of unrelated and the other three stance can be seen as samples drawn from two different semantic distributions. In order to measure the discrepancy between these two distributions, we employ the Maximum Mean Discrepancy (MMD) [176] based on the learnt sample representation $\mathbf{u}$ from two distributions. It does not involve density estimation, and has been reported to achieve success in face recognition and image annotation [177]. We give its definition followed by practical implementations. 
76 Chapter 4. Hierarchical Stance Representation and Detection for Online News

Definition 1. Maximum Mean Discrepancy [176]: "Let $p$ and $q$ be two Borel probability distributions over a space $\mathcal{X}$ and let $X$ and $Z$ be sets with independent identically distributed samples drawn from $p$ and $q$. The MMD is defined by a class $\Psi$ of map functions $\psi: \mathcal{X} \rightarrow \mathcal{H}$ as:

$$
M M D(p, q, \Psi)=\sup _{\psi \in \Psi}\left(\mathbf{E}_{p}[\psi(x)]-\mathbf{E}_{q}[\psi(z)]\right)
$$

Here, $x$ and $z$ are samples from $X$ and $Z$."

In other words, the MMD equation defines the largest possible distance between two expectations over the set of functions $\Psi$. Moreover, it has been discussed in $[176,178]$ that "when $\mathcal{H}$ is the reproducing kernel Hilbert space (RKHS) [179], this means that for all $x \in \mathcal{X}$, the linear point evaluation function mapping $\psi \rightarrow \psi(x)$ exists and is continuous. When $\Psi$ is the unit ball in a universal RKHS, it is guaranteed that $M M D(p, q, \Psi)$ will detect any discrepancy between $p$ and $q$." Hence we use a set of linear functions $\Psi$.

Specializing to the task of news stance detection, we let $p$ denote the distribution for the unrelated hidden representations in our model, with sample set $\mathcal{U}^{1}=\left\{\mathbf{u}_{1}^{1}, \ldots, \mathbf{u}_{n_{1}}^{1}\right\}$ and according to Eq. (4.1) their generating input set $\mathcal{V}^{1}=\left\{\mathbf{v}_{1}^{1}, \ldots, \mathbf{v}_{n_{1}}^{1}\right\}$. And, $q$ denotes the distribution for the related samples (including agree, disagree and discuss hidden representations), with sample set $\mathcal{U}^{2}=\left\{\mathbf{u}_{1}^{2}, \ldots, \mathbf{u}_{n_{2}}^{2}\right\}$ and according to Eq. (4.1) their generating input set $\mathcal{V}^{2}=$ $\left\{\mathbf{v}_{1}^{2}, \ldots, \mathbf{v}_{n_{2}}^{2}\right\} . n_{1}$ and $n_{2}$ are the number of samples in $\mathcal{U}^{1}$ and $\mathcal{U}^{2}$ respectively. Thus we have $\mathcal{X}=\mathcal{R}^{k}$ and $\mathcal{H}=\mathcal{R}^{j}$. The function $\psi$ is implemented as a linear kernel $\psi(x)=\theta_{d} x$, where $\theta_{d}$ is a $j \times k$ matrix in the projection layer. $k$ and $j$ are the space dimensions. According to Eq. (4.1), the hidden representation $\mathbf{u}$ is parameterized by $\theta_{u}$, thus the empirical expression of MMD is parameterized by $\theta_{u}$ and $\theta_{d}$ :

$$
\begin{aligned}
d\left(\theta_{u}, \theta_{d}\right) & =\frac{1}{n_{1}} \sum_{i=1}^{n_{1}} \theta_{d} \mathbf{u}_{i}^{1}-\frac{1}{n_{2}} \sum_{i=n_{1}+1}^{n_{1}+n_{2}} \theta_{d} \mathbf{u}_{i}^{2} \\
& =\frac{1}{n_{1}} \sum_{i=1}^{n_{1}} \theta_{d} f\left(\mathbf{v}_{i}^{1} ; \theta_{u}\right)-\frac{1}{n_{2}} \sum_{i=n_{1}+1}^{n_{1}+n_{2}} \theta_{d} f\left(\mathbf{v}_{i}^{2} ; \theta_{u}\right)
\end{aligned}
$$


We assume the larger the MMD term is, the easier it is to distinguish the unrelated from the related (actually consisting of three different stance) samples. By constantly changing the projection layer parameterized by $\theta_{d}$, we find the maximum expectation difference between the data representations of unrelated and the other three stances. With the MMD regularization term in the whole loss function, data representations can be modified in favour of related/unrelated classification and agree/disagree/discuss classification.

\subsubsection{Optimization}

In order to make the distributions easier to be distinguished, a larger MMD regularization term is preferred, and we treat the regularization term as an extra goal besides classification. We integrate the two-layer classification loss (see Eq. (4.8)) and use MMD as a regularization term (see Eq. (4.10)) into a single objective function $(L)$. Specifically, we add these two sub-goals with a hyperparameter $\beta$ as follows:

$$
L\left(\theta_{u}, \theta_{r}, \theta_{s}, \theta_{d}\right)=l^{c}\left(\theta_{u}, \theta_{r}, \theta_{s}\right)-\beta \cdot d\left(\theta_{u}, \theta_{d}\right),
$$

where $\beta$ leverages the importance of the regularization. The larger the MMD regularization term is, the easier is for the classifier to distinguish between the related and unrelated stances. Thus, the sign of the regularization term is negative.

The optimization involves the minimization of the classification loss $L$ with respect to $\theta_{u}, \theta_{r}, \theta_{s}$, and $\theta_{d}$ as follows:

$$
\min _{\theta_{u}, \theta_{r}, \theta_{s}, \theta_{d}} L\left(\theta_{u}, \theta_{r}, \theta_{s}, \theta_{d}\right)
$$

Optimizing the model consists of two sub-goals. On the one hand, we want to maximize the distribution discrepancy between the two classification layers. On the other hand, we want to minimize the classification loss of both layers. Both of these two sub-goals involve the feature layer parameter $\theta_{u}$ update, but in opposite update directions. The optimization process will not stop until a saddle point (the feature layer parameters can be well applied in both sub-goals) is reached. 
78 Chapter 4. Hierarchical Stance Representation and Detection for Online News

\subsubsection{Prediction}

Given as input a feature vector $\mathbf{v}$, the classifier outputs the following probabilities: $p$ (unrelated $), p($ agree $\mid$ related $), p($ disagree $\mid$ related $)$, and $p($ discuss $\mid$ related $)$. However, these last 3 probabilities are not comparable with the first one. To make them comparable we derive $p$ (agree), $p$ (disagree) and $p$ (discuss). By observing that the class agree is assumed as related, thus $p($ agree, related $)=p($ agree $)$, we derive that:

$$
\begin{aligned}
p(\text { agree }) & =p(\text { agree }, \text { related }) \\
& =p(\text { agree } \mid \text { related }) \times p(\text { related }) \\
& =p(\text { agree } \mid \text { related }) \times(1-p(\text { unrelated })) .
\end{aligned}
$$

Similarly, for the other two classes we derive that:

$$
\begin{gathered}
p(\text { disagree })=p(\text { disagree } \mid \text { related }) \times(1-p(\text { unrelated })), \\
p(\text { discuss })=p(\text { discuss } \mid \text { related }) \times(1-p(\text { unrelated })) .
\end{gathered}
$$

Thereby, the model actual output $\hat{\mathbf{y}}$ is:

$$
\hat{\mathbf{y}}=(p(\text { agree }), p(\text { disagree }), p(\text { discuss }), p(\text { unrelated })),
$$

where the class with the highest probability corresponds to the predicted stance. Algorithm 1 shows the parameter update process, which is based on the mini-batch gradient descent algorithm. 


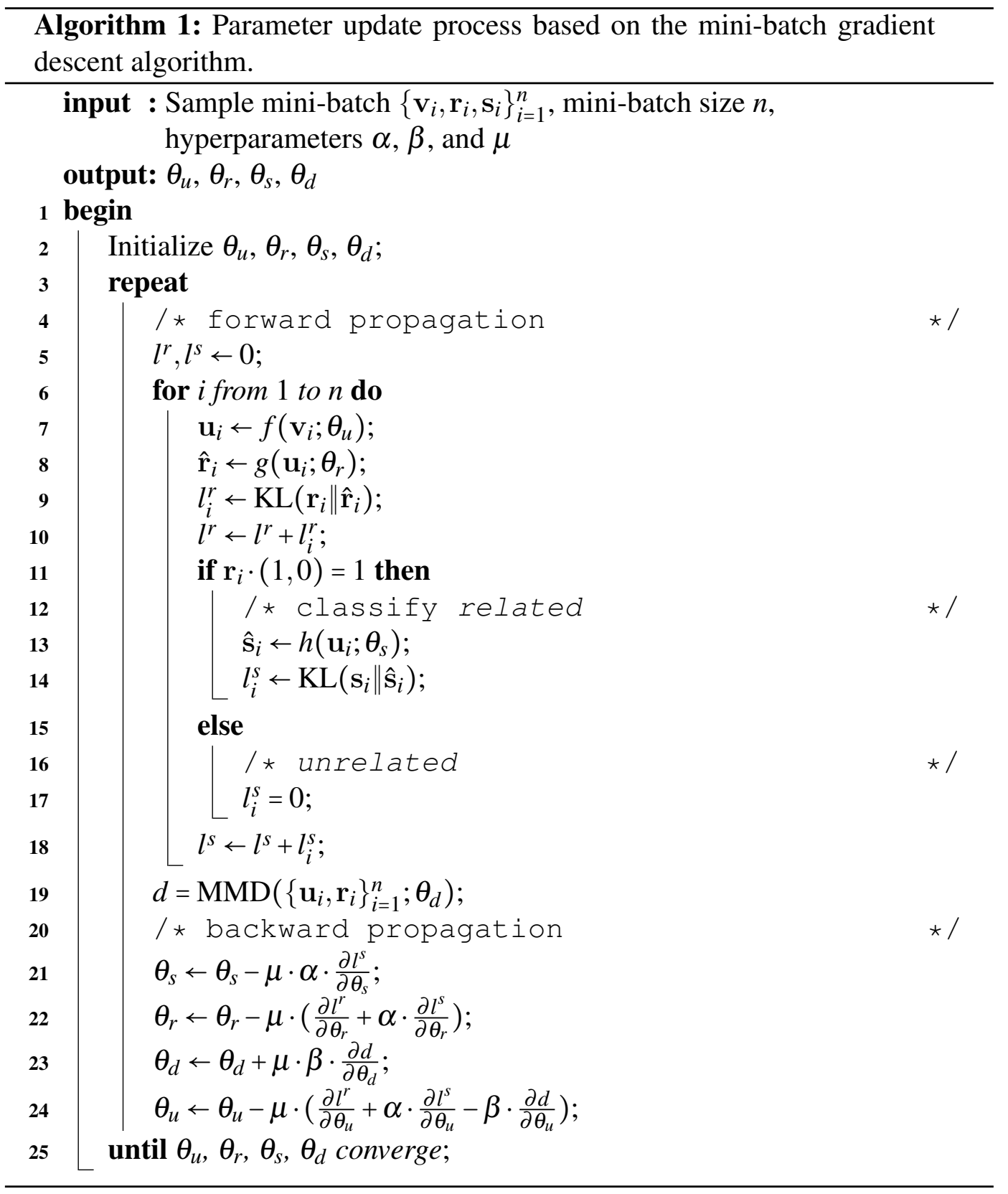


80 Chapter 4. Hierarchical Stance Representation and Detection for Online News

\subsection{Experimental Setup}

We start this section by presenting the datasets and evaluation measures relevant to the news stance detection task. Then, we describe the features used by our model and the model parameterization. Finally, we present the baselines.

Experiments are conducted on two publicly available datasets: the Emergent dataset $^{2}$ [111] and the $F N C-1$ dataset $^{3}$. In these two datasets, a claim consists of a news article headline and of a news article content. These datasets are split into train and test subsets; see Table 4.1 for statistics about the splits. Hyper-parameters are tuned on the validation set split from the training subset.

The FNC-1 dataset consist of 75,385 instances. Each instance in the dataset is a pair claim-text labeled as one of the four stances: agree, disagree, discuss and unrelated. The ratio of training data over testing data in the FNC-1 dataset is $~ 2: 1$. Every class accounts for a similar percentage in the train and test subsets. The unrelated stances are the majority (over 70\%) in both subsets, while the disagree stances are less than 3\%. The agree and discuss stances are less than $20 \%$ and $10 \%$.

The Emergent dataset is similar to the FNC-1 dataset, however it contains only agree, disagree and discuss stances. Hence, it needs to be augmented with unrelated stances. Similarly to how the FNC-1 dataset unrelated stances have been labeled, we manually labeled unrelated stances by pairing a claim with an unrelated text, i.e., paired with another claim. Moreover, to make the class distributions less imbalanced, we make the ratio of related stances and unrelated ones $\sim 1: 1$. The augmented Emergent dataset contains 4,071 training labels and 1,024 testing labels with a ratio of $\sim 4: 1$. Class distributions between train and test subsets are similar.

\subsubsection{Research Questions}

To validate the effectiveness and facilitate the understanding of the hierarchical stance detection model, we seek to answer the following four research questions, which will be guide the remainder of the chapter:

RQ1 What is the overall performance of the proposed model against baselines?

\footnotetext{
${ }^{2}$ https://github.com/willferreira/mscproject.

${ }^{3}$ https://github.com/FakeNewsChallenge/fnc-1.
} 
Table 4.1: Statistics of the datasets.

\begin{tabular}{lccccc}
\hline \multirow{2}{*}{ Subset } & Stance & \multicolumn{2}{c}{ Emergent } & \multicolumn{2}{c}{ FNC-1 } \\
& & Number & Percentage & Number & Percentage \\
\hline \hline Training & agree & 992 & 24.37 & 3,678 & 7.36 \\
& disagree & 303 & 7.44 & 840 & 1.68 \\
& discuss & 776 & 19.06 & 8,909 & 17.83 \\
& unrelated & 2,000 & 49.13 & 36,545 & 73.13 \\
\hline \hline Testing & agree & 4,071 & & 49,972 & \\
& disagree & 916 & 24.02 & 1,903 & 7.49 \\
& discuss & 776 & 19.89 & 697 & 2.74 \\
& unrelated & 500 & 48.83 & 4,464 & 17.57 \\
& & & 18,349 & 72.20 \\
\hline
\end{tabular}

RQ2 Do the hierarchical architecture and the MMD regularization improve the model performance?

RQ3 How does the dimension of the hyper-parameter $\alpha$ and $\beta$ affect model's performance?

RQ4 What is the contribution of the used features in classifying stance?

\subsubsection{Datasets}

Compared to the FNC-1 dataset, the class distributions of the augmented Emergent dataset is more balanced. The percentage of unrelated stances is about $50 \%$, whereas the percentages of agree and disagree stances are about $24 \%$ and $8 \%$. Both datasets have similar percentages of the discuss stances.

\subsubsection{Evaluation Measure}

In line with the FNC-1 challenge, the evaluation is based on a weighted two-level scoring system based on the accuracy measure. This evaluation measure, called relative score, evaluates a model by splitting the stance detection task into two subtasks, related/unrelated and agree/disagree/discuss classification sub-tasks. To the former sub-task is given a $25 \%$ weight. This is done because this sub-task is considered to be easier than the latter sub-task to which is given a $75 \%$ weight.

We report the evaluation measures: relative score, accuracy, and accuracy on a per class basis. 


\subsubsection{Feature Extraction}

To represent claims and texts we use a bag-of-words approach. For each claim and text we generate a TF-IDF vector, and for each pair claim-text we compute their cosine similarity. We also include the FNC-1 official features into the input feature vector.

The final set of features include:

- TF-IDF vectors of claims;

- TF-IDF vectors of texts;

- Cosine similarity (CosSim) between the claim vector and the text;

- Ratio of word overlap (WordLap) between the claim and the text;

- An Indicator whether a claim has refuting words (RefWord);

- The polarity (Pol) of the claim and the text;

- The number of overlapping $n$-grams (NGrams) for $n \in\{2,3,4,5,6\}$ between the claim and the text.

For the TF-IDF vectors, we only use the top 2,000 most frequent terms except stopwords. All of these features are concatenated to form the input feature vector $\mathbf{v}$. Compared to conventional stance detection discussed before, we did not use pretrained word embeddings in the input features, which is because they lead to decreased performance during model evaluation.

\subsubsection{Experimental Setting}

The following hyperparameters have been set via a five-cross validation on the train subsets:

- The dimension $k$ of hidden representations is set to 100 ;

- The dimension $j$ of the MMD is set to 10 ;

- The activation function used in the hidden layers is set to ReLu; 
- The parameters $\alpha$ are set to 1.5 and 1.3 for the Emergent and FNC-1 datasets.

- The parameter $\beta$ is set to 0.001 ;

The function $f$ and $g$ in (4.1), (4.2) and (4.3) are implemented as multi-layer perceptrons (MLP). We include a L2 regularization term [180] for the MLP weight parameters in the final loss function to mitigate overfitting. Dropout is also used to mitigate overfitting with rate set to 0.6. We train in mini-batches of size 64 over the entire train subset. Note that the gradient steps in Algorithm 2 can easily be alternated with a more powerful optimizer such as the Adam optimizer [181]. Early stopping is applied when the classification loss on the validation subset does not get smaller for three continuous iterations. The whole model is implemented with TensorFlow.

\subsubsection{Baselines}

We compare our model against the baseline methods. These methods are chosen because they either performed well in the Fake News Challenge in 2017 or is a follow-up work with improved performance published later. Among them we distinguish between methods that use the same features as ours and methods that learn their representations. We start with the latter type, we call these representation learning-based baselines:

Bidirectional LSTM (BiLSTM). [182] uses bidirectional LSTM: a LSTM encodes the claim, and the other encodes the text with the encoded claim set as initial states.

Attentive CNN (AtCNN). [173] builds an attention-augmented CNN. The claim and the text are input to a convolutional neural network to obtain hidden representations, and the attention mechanism is employed to locate the most influential words or phases on the final results;

Memory Network (MN). [183] develop an end-to-end memory network for stance detection. The network operates at the paragraph level and integrates 
84 Chapter 4. Hierarchical Stance Representation and Detection for Online News convolutional and recurrent neural networks, as well as a similarity matrix as part of the overall architecture;

Ranbking Model (RM). [20] build a ranking method to tackle the stance detection and achieve empirical performance improvements. A ranking loss function is proposed to replace Softmax and maximize the representation difference between four classes of stance.

We now review the second type of baselines: those methods that use the same features as our method, we call these feature engineering-based baselines:

Official Baseline (OB). This is the FNC-1 official baseline that uses one gradient boosting decision trees model for four-way classification;

Logistic Regression (LR). [167] use $n$-gram matching and a rule-based procedure to decide relatedness, and three-way logistic regression to distinguish among the related classes;

Gradient Boosted Decision Trees (GBDT). [169] develop two GBDT models, one to determine the relatedness of an text to a claim, and another to distinguish among the related classes;

Multi-Layer Perception (MLP). This model [91] achieved the third best performance in FNC-1. It extracts TF-IDF and cosine similarity between claims and texts as input features, and uses a MLP as the four-class classifier.

\subsection{Results and Discussion}

In this section, we start by analyzing the dependency assumption. Then, we compare and contrast our model against the baselines. Next, we provide a sensitivity analysis of the hyperparameters. We conclude with an impact analysis of the features used by the model.

\subsubsection{Overall Performance (RQ1)}

In Tables 4.2 and 4.3 we compare our model against the state-of-the-art models. Our model achieves the best stance detection performance for the relative score on 
Table 4.2: Performance comparison of our model against the State-of-the-Art models on the augmented Emergent dataset.

\begin{tabular}{|c|c|c|c|c|c|}
\hline \multirow[t]{2}{*}{ Model } & \multicolumn{4}{|c|}{ Accuracy $(\%)$} & \multirow{2}{*}{$\begin{array}{c}\text { Relative Score } \\
(\%)\end{array}$} \\
\hline & agree & disagree & discuss & unrelated & \\
\hline \multicolumn{6}{|c|}{ Feature Engineering-Based Baselines } \\
\hline OB & 33.56 & 23.44 & 70.23 & 84.00 & 74.86 \\
\hline LR ( [167]) & 66.73 & 40.51 & 78.33 & 78.00 & 83.45 \\
\hline GBDT ( [169]) & 80.62 & 50.42 & 83.52 & 88.00 & 87.53 \\
\hline $\operatorname{MLP}([91])$ & 58.53 & 23.64 & 79.05 & 95.00 & 85.43 \\
\hline RM ( [20]) & 64.56 & 40.42 & 85.45 & 96.00 & 87.69 \\
\hline \multicolumn{6}{|c|}{ Representation Learning-Based Baselines } \\
\hline BiLSTM ( [182] ) & 43.21 & 12.57 & 78.55 & 96.00 & 81.37 \\
\hline $\operatorname{AtCNN}([173])$ & 44.78 & 14.60 & 72.44 & 97.00 & 83.56 \\
\hline MN ( [183]) & 54.64 & 40.05 & 72.10 & 89.00 & 85.92 \\
\hline \multicolumn{6}{|l|}{$\overline{\text { Our Models }}$} \\
\hline Independent & 74.54 & 45.32 & 82.59 & 95.49 & 86.33 \\
\hline Dependent & 63.54 & 44.68 & 68.35 & 95.00 & 86.72 \\
\hline Learned & 82.52 & 69.05 & 84.30 & 97.00 & 89.30 \\
\hline
\end{tabular}

Table 4.3: Performance comparison of our model against the State-of-the-Art models on the FNC-1 dataset.

\begin{tabular}{|c|c|c|c|c|c|}
\hline \multirow[t]{2}{*}{ Model } & \multicolumn{4}{|c|}{ Accuracy $(\%)$} & \multirow{2}{*}{$\begin{array}{c}\text { Relative Score } \\
(\%)\end{array}$} \\
\hline & agree & disagree & discuss & unrelated & \\
\hline \multicolumn{6}{|c|}{ Feature Engineering-Based Baselines } \\
\hline $\mathrm{OB}$ & 10.51 & 1.00 & 79.66 & 97.98 & 75.20 \\
\hline $\operatorname{LR}([167])$ & 67.42 & 31.61 & 75.23 & 95.36 & 80.63 \\
\hline GBDT ( [169]) & 82.93 & 69.82 & 33.52 & 95.42 & 86.72 \\
\hline $\operatorname{MLP}([91])$ & 44.04 & 6.60 & 81.38 & 97.90 & 81.72 \\
\hline $\mathrm{RM}([20])$ & 64.90 & 27.26 & 84.41 & 99.12 & 86.66 \\
\hline \multicolumn{6}{|c|}{ Representation Learning-Based Baselines } \\
\hline BiLSTM ( [182]) & 35.96 & 0.94 & 80.33 & 98.54 & 78.70 \\
\hline $\operatorname{AtCNN}([173])$ & 38.67 & 8.24 & 70.63 & 91.25 & 75.77 \\
\hline MN ( [183]) & 16.92 & 60.22 & 81.27 & 95.50 & 79.92 \\
\hline \multicolumn{6}{|l|}{ Our Models } \\
\hline Independent & 72.41 & 37.90 & 68.23 & 97.43 & 83.47 \\
\hline Dependent & 61.34 & 42.93 & 59.38 & 99.05 & 85.32 \\
\hline Learned & 80.61 & 72.35 & 77.49 & 99.53 & 88.15 \\
\hline
\end{tabular}

both datasets. The model achieves $89.30 \%$ on the augmented Emergent test subset and $88.15 \%$ on the FNC-1 test subset.

By comparing with four-way classification baselines (OB, MLP, BiLSTM, AtCNN, MN and RM) we demonstrate the advantage of separating the relatedness detection from the stance detection. We observe that these classifiers perform 
poorly on the disagree class, which is caused by the large percentage difference between the minority disagree class and the majority unrelated class. Further, the more imbalanced the evaluation dataset becomes, the worse performance the fourway classifiers achieve on the minority disagree class.

By comparing with baselines that separate the relatedness detection from the stance detection (LR and GBDT) we demonstrate the superiority of a single end-toend model. Removing the regularization term from our model reveals contribution of the regularisation to model's performance. Specifically, removing the regularization term leads to decreased detection performance for all the four stances.

Figure 4.2 shows the confusion matrix. Here we observe the detection performance on a per class basis. For the related/unrelated classification, we correctly classify $97.00 \%$ and $99.53 \%$ unrelated instances on the augmented Emergent and the FNC-1 test subsets. There is some misclassification between the agree and unrelated classes, and between the discuss and unrelated classes. The misclassification of the disagree class accounts for the largest error of the unrelated instances.
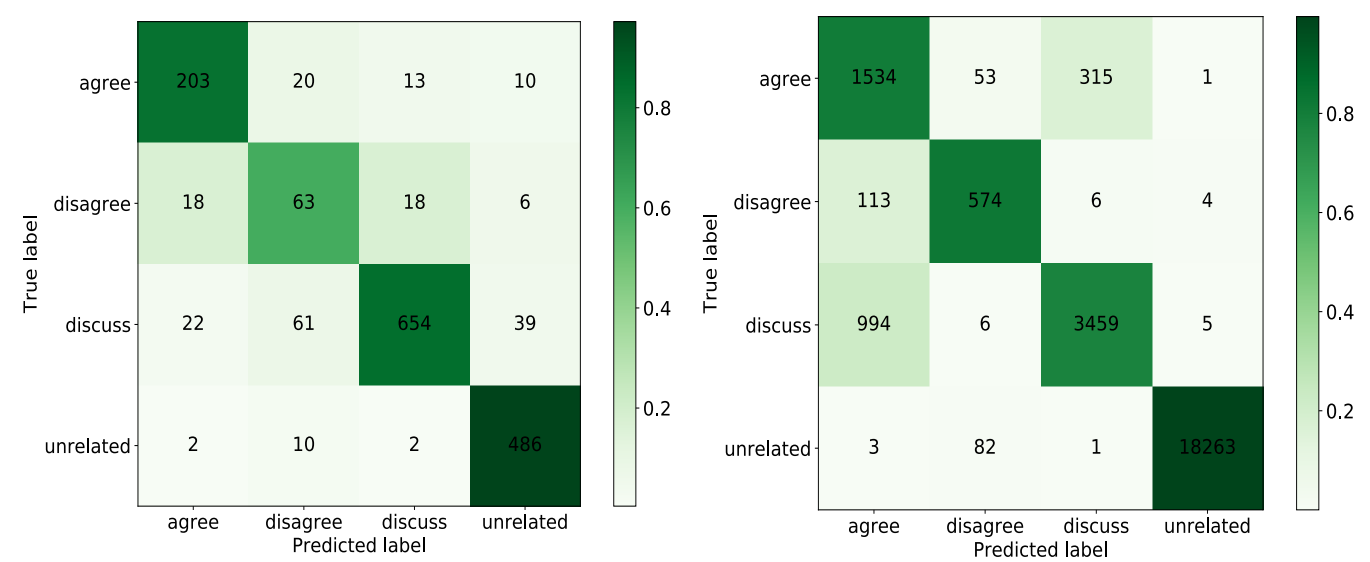

Figure 4.2: The confusion matrices of our model for the augmented Emergent (on the left) and FNC-1 (on the right) datasets.

Our model achieves an accuracy of $69.05 \%$ and $72.35 \%$ for the disagree class on the Emergent and the FNC-1 test subsets. The classification accuracy is largely improved compared to the state-of-the-art. Some misclassification error exists between agree and disagree. However, our model can distinguish between the discuss and the disagree with few errors. While the number of discuss cases is the largest 
and the number of disagree instances is the smallest, our model does not mistake disagree instances as discuss ones, i.e., the model has learned the core representation difference between these two classes. Due to ambiguous expressions, misclassification between agree and discuss is the cause of most errors between these classes, which leads to a slightly worse accuracy for the discuss class on the Emergent $(84.30 \%)$ and FNC-1 (77.49\%) test subsets.

Two reasons account for the improved empirical performance observed on our model. On the one hand, the mitigation of the class imbalance problem. Contrary to the four-way classifiers that directly compare the disagree and unrelated instances, the hierarchical model avoids the direct comparison of this minority disagree class (less than $2 \%$ in the FNC-1 dataset) with the majority unrelated one (over $70 \%$ in the FNC-1 dataset). On the other hand, the MMD term that maximizes the discrepancy between the unrelated class and the aggregated related classes. Since the agree, disagree and discuss belong to the same class, the related class, the MMD regularization promotes the emergence of features that are useful to separate the class pairs: agree with unrelated, disagree with unrelated, and discuss with unrelated.

\subsubsection{Dependency Assumption (RQ2)}

In Figure 4.3 we show the effect of the 3 dependency assumptions by visualizing the learned representations using a t-SNE projection [184]. We observe that when the classifiers are assumed independent, i.e., the classification is performed in cascade — no error is propagated from the second layer to the first during training — then the learned representation well separates the unrelated class from the unrelated ones. When the classifiers are assumed dependent, i.e., the two classifiers are trained together - the error is left free to propagate from the second layer to the first - then the learned representation is not very well separated. However, when the dependence assumption of the two classifiers is learned via the MMD regularization, i.e., the two classifiers are trained together with the error propagation controlled by the regularizer, then the learned representation is again well separated like in the first case. Well-separated representations suggest a greater discriminative power of the model — the unrelated and related classes are almost linearly separable. 


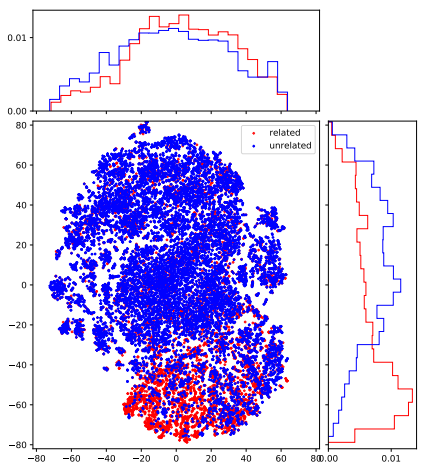

(a) Independent

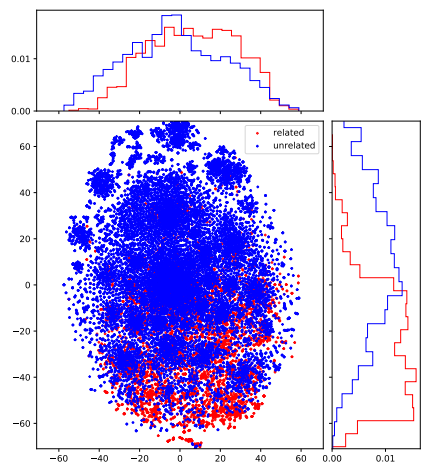

(b) Dependent

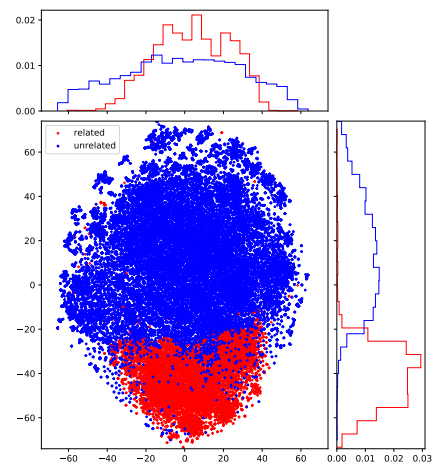

(c) Learned

Figure 4.3: t-SNE visualization of the hidden representations on the training data. Blue points denote hidden representations of unrelated samples from the first layer while red points denote those of stance samples from the second layer. The curves indicate the distribution of data points when projected to the onedimensional space (the $x$-axis and the $y$-axis). The hidden representations of model trained (a) with separated layers (b) together but without regularization, and (c) with MMD regularization.

The last three rows of Tables 4.2 and 4.3 show the performance of our model on the two test subsets for each one of the three assumptions: independent, dependent, and learned. Looking at the accuracy of the unrelated class, we observe that the accuracy is greater when the learned representations are well-separated, as in the independent and learned cases. Furthermore, looking at all the other scores, we observe the learned assumption outperforms both the independent and dependent assumptions in all other cases, demonstrating that learning together both, relatedness and stance of texts towards claims, is beneficial to the stance detection task.

\subsubsection{Hyper-parameter Sensitivity (RQ3)}

In this subsection we discuss the sensitivity to the hyperparameters of our model. The most influential hyperparameters for the proposed model are $\alpha$ and $\beta$. The former controls the relative importance of classification layers. The latter leverages the regularization.

In Figures 4.4(a) and 4.4(b) we show how the performance of the model changes when varying $\alpha$ and $\beta$ for the augmented Emergent and FNC-1 test subsets. $\alpha$ is searched between 0.1 and 3.0 with steps of 0.1 , and $\beta$ is searched in 


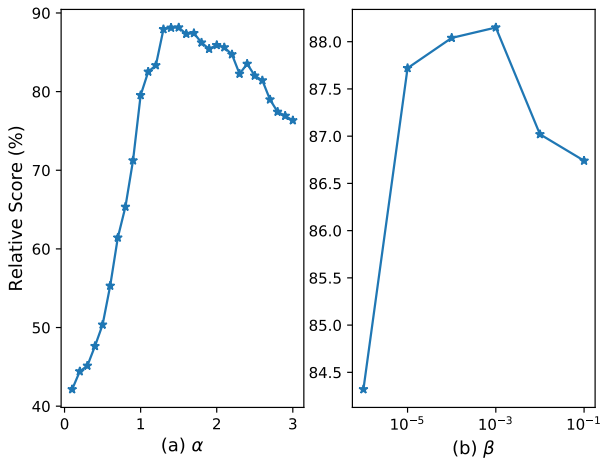

(a) Emergent
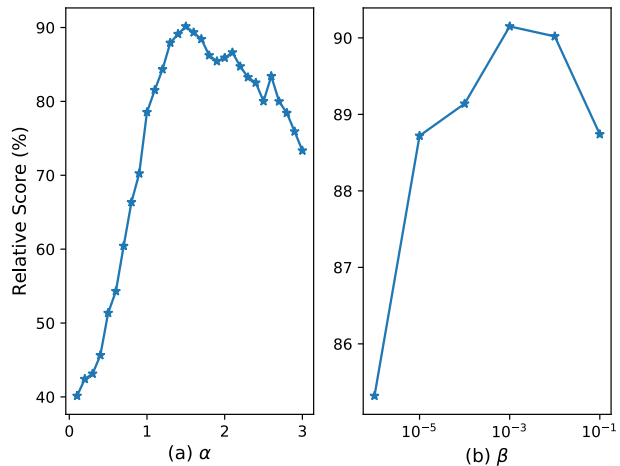

(b) FNC-1

Figure 4.4: Sensitivity of the trained model when varying $\alpha$ and $\beta$ on the test subset of the augmented Emergent (on the left) and FNC-1 (on the right) datasets.

Table 4.4: Performance of our model with different feature sets on the FNC-1 dataset. "”" denotes no feature set is removed.

\begin{tabular}{lcccc}
\hline Removed Feature Set & \multicolumn{3}{c}{ Accuracy $(\%)$} \\
& agree & disagree & discuss & unrelated \\
\hline \hline CosSim & 71.53 & 85.08 & 78.76 & 69.37 \\
WordLap & 67.43 & 80.49 & 77.31 & 77.89 \\
RefWord & 74.43 & 64.37 & 77.03 & 97.49 \\
Pol & 60.49 & 67.93 & 80.92 & 98.79 \\
NGrams & 74.27 & 75.73 & 87.82 & 84.52 \\
/ & 80.61 & 82.35 & 77.49 & 99.53 \\
\hline
\end{tabular}

$\{0,0.1,0.01,0.001,0.0001,0.00001\}$. For $\alpha$, we observe that the performance of the model improves quickly as $\alpha$ increases and peaks at 1.5 and 1.3 for the FNC-1 and augmented Emergent datasets, then the performance experiences a slight decrease when $\alpha$ is increased. We hypothesize that the optimal $\alpha$ is related to the class balance between the unrelated class and the related ones. The more unbalanced the dataset is towards the unrelated class, larger is the optimal $\alpha$. For $\beta$, we observe that the performance is the highest when $\beta$ is set to 0.001 . This happens for both augmented Emergent and FNC-1 test subsets. These optimal values of $\alpha$ and $\beta$ observed on the test subsets are equal to the one found when training the model.

\subsubsection{Feature Analysis (RQ4)}

In this subsection we evaluate and discuss the importance of each feature towards the final prediction. To examine the influence of each feature on the final performance, we do a leave-one feature set-out approach and record the classification 
accuracy on the stance detection task. The following analysis is only based on the FNC-1 dataset. Similar results are observed on the augmented Emergent dataset.

In Table 4.4 we show the results of this analysis. We observe that removing the Cosine Similarity (CosSim) feature leads to a large decrease in accuracy for the unrelated class. Similarly, the use of Ratio of Word Overlap (WordLap) has a positive effect for the agree class, and it also contributes to the unrelated class. The Refuting Words (RefWord) and Polarity (Pol) features help for the classes agree and disagree, while removing the Overlapping $n$-Grams (NGram) feature leads to an increase on the discuss class, i.e., the NGram feature causes confusion between the discuss and the other classes.

\subsection{Discussion}

\subsubsection{Hierarchy and MMD}

Hierarchy is very common in the nature and the human society. Conceptualized objects are often hierarchically organized with different abstraction granularities. To develop human-like intelligence, machine learning models are supposed to efficiently recognize and deal with objects at different abstraction levels, i.e., in a hierarchical way [185]. In unsupervised learning, researchers have proposed hierarchical clustering to group objects into a hierarchy of clusters based on their mutual similarity, which has proved to produce more accurate results [186] than flat clusters. As for supervised classification, hierarchical machine learning can be implemented as multi-level classifiers by integrating features from different grain-sizes, e.g., the coarse, the middle-level and the fine. This has been studied in wide areas such as image retrieval [187]. Another typical case is the Hierarchical softmax (H-Softmax), an approximation to the softmax function, which is inspired by binary trees and used to predict words in neural language models [188]. With a similar multi-level learning strategy, the hierarchical stance detection model allows us to decompose a complex task, e.g., the four-way news stance classification, into sub-tasks at different abstraction levels, e.g., the related/unrelated and agree/disagree/discuss classifications, so as to utilize different grain-sized information. 
An additional benefit of hierarchical classification is the potential to alleviate the class imbalance problem. In the hierarchical setting, data of different classes can form a new class at a more abstract level with a bigger group. In our experimental datasets, data at the fine-grained level (i.e., agree, disagree and discuss) are from the minority classes, and constitute a larger group (i.e., the new related class), which balances the ratio of class sizes over the unrelated class. This benefit may not generalize to other hierarchical cases because the constituted larger group in the coarse layer (i.e., the up-level layer) can worsen the ratio of class dataset sizes when the majority is at the fine-grained level.

The MMD regularization term modifies the learnt data representations before they are input to the multi-level classifiers. What it does during the optimization of the objective function is to try to enlarge the discrepancy between representations of unrelated samples and the other three types of stance. Such behaviour improves the overall classification performance, because enlarging the discrepancy makes it easier for the first-level classification based on just coarse representations, and then fine-grained representations are learnt in the second-level classification. The rational behind this is that the three fine-grained stances have a conceptual abstraction - related, which is exactly opposed to unrelated. Verifying whether MMD can be generalized to all hierarchical machine learning is out of the scope of this thesis, but we suspect this generalization may not hold at least for some cases. For example, H-Softmax does not predict words based on conceptual abstraction at different granularities, thus may not benefit from MMD. Other tasks of stance detection, such as those on the social media (e.g., the Pheme dataset), may not experience such a benefit as well, because the stance classes, i.e., supporting, denying, commenting and questions, are not semantically hierarchically distributed and thus there does not exist a semantically hierarchical structure.

We do not assume that MMD contributes to the alleviation of class imbalance. What MMD influences is the learnt representations of the data input. It cannot balance the ratio of class sizes or add penalty to misclassified minority data, the two ways of which are commonly used to solve the class imbalance problem [189]. To 
verify this assumption, one can compare a hierarchical machine learning model on a balanced dataset, with and without the MMD regularization term.

\subsubsection{Model Deployment}

The hierarchical stance detection can be readily deployed by news websites. It extracts common features as the input, such as TF-IDF, $n$-grams and polarity indicators. The main modules are implemented by common deep learning modules including multi-layer perceptron and the regularization term is implemented as matrix multiplication. These make the computational complexity at an acceptable level. One more benefit is that, as the experiment results show, the proposed model is not sensitive to hyper-parameters such as $\alpha$ and $\beta$ that determine the loss function. This means the model will not be influenced too much when the proportions of stance classes are moderately different from the training datasets.

One minor issue is that the TF-IDF values need to be re-calculated as more articles are available in the corpus. Otherwise, the proposed model may be biased in terms of the words used in an article.

\subsection{Summary}

In this chapter, we study the problem of news stance detection: the classification of the stance of a piece of text towards a claim into one of the four classes: agree, disagree, discuss and unrelated. We observe that the baselines perform poorly on the disagree class, which is caused by the large percentage difference between the minority disagree class and the majority unrelated class. Further, the more imbalanced the evaluation dataset becomes, the worse performance the four-way classifiers achieve on the minority disagree class. By comparing with baselines that separate the relatedness detection from the stance detection (LR and GBDT) we demonstrate the superiority of a single end-to-end model. LR and GBDT are better on the disagree class, although their overall performance is worse than our model. By comparing with four-way classification baselines we demonstrate the advantage of separating the relatedness detection from the stance detection.

Two reasons account for the improved empirical performance observed on our 
model. On the one hand, the mitigation of the class imbalance problem. Contrary to the four-way classifiers that directly compare the disagree and unrelated instances, the hierarchical model avoids the direct comparison of this minority disagree class with the majority unrelated one. The disagree class is separated with the agree and discuss class, in which case the instance number ratio of disagree over discuss is 4 times larger than that of disagree over unrelated in the FNC-1 dataset. On the other hand, the MMD term that maximizes the discrepancy between the unrelated class and the aggregated related classes. Since the agree, disagree and discuss belong to the same class, the related class, the MMD regularization promotes the emergence of features that are useful to separate the class pairs: agree with unrelated, disagree with unrelated, and discuss with unrelated. 



\section{Chapter 5}

\section{User Replies Aided Misinformation}

\section{Detection}

Besides news articles studied in last chapter, people often express their stance in social media by writing comments and replies towards a claim. For newly emerging topics and events, long well-formulated articles are usually limited or even not available for a certain period. In contrast, opinions from multiple users and sources in social media provide instant evidential signals when it comes to identifying the truthfulness of an emerging claim [17]. For example, false information often triggers versatile, mostly controversial discussion (involving the stance of agree and disagree) among the public. In this chapter, we study how to utilize user replies towards a claim in social media to predict veracity.

Researchers have studied to utilize evidential signals from social media to detect false information. The SemEval-2017 Task 8 defines a research task where participants aim to verify "rumours in the form of claims made in user-generated content" [94] in social media. The Task 8 involves two subtasks: (1) stance detection of tweets towards claims in the context of Twitter, and (2) veracity classification of the claim at question. The stance detection of tweets has different class definition from news stance: a new questioning is added while unrelated does not exist. In terms of veracity classification, there are two settings: (a) the closed setting requires to predict veracity solely based on the claim itself and (b) the open setting provides access to the claim context including an additional Wikipedia dump 
and user replies. Models developed in the closed setting is quite similar to the content-based approach we discuss in Chapter 2, while the open setting equipped with Wikipedia and user replies can fall into the knowledge-based and the social context-based approaches respectively.

\subsection{Problem Statement}

For the sake of clarity, we specify the research task and define relevant symbols used in this chapter. The task of veracity evaluation is to predict the truthfulness of textual claims, i.e., social posts in this chapter, given their content and people's replies in social media [190].

Let $\mathcal{C}=\left\{c_{1}, c_{2}, \ldots, c_{N}\right\}$ be a set of $N$ textual claims. The claim $c_{i}$ is instantiated as a post in social media and commented by a set of $M$ user replies $\mathcal{D}_{i}=\left\{d_{i, 1}, d_{i, 2}, \ldots, d_{i, M}\right\}$. These replies are temporally ordered with the last one being latest, and they usually constitute a conversation [25]. For example, in Figure 5.1 we show a false claim posted on Twitter about an alleged shooting in Ottawa. There are a number of user replies towards this post. We use $y_{i}$ to denote the binary veracity label of the claim $c_{i}$, which could be either $y_{i}=1$ for true or $y_{i}=0$ for false. We do not take the unverified case into consideration of the veracity classes, because we set out to simplify the research task and focus on the pattern difference of true and false claims.

The tuple of a claim and people's replies, i.e., $\left\{c_{i}, \mathcal{D}_{i}\right\}$, forms a data instance to predict the claim veracity $y_{i}$. For the sake of clarity, in the following, we will omit the subscripts $i$ when describing a single instance: $\{c, \mathcal{D}, y\}$.

\subsection{Motivation}

False information content or social context can be ambiguous in the raw semantic space. Most existing models employ feature engineering or deep learning to extract features from claims' content and people's replies. These models produce deterministic mappings to capture the difference between true or false claims. A major limitation of these models is their inability to represent ambiguity of semantics. It has been pointed out that latent distributions help discriminative deep learning 
Police have clarified that there were two shootings in Ottawa today, not three: at the War Memorial and Parliament Hill.

11:26 AM - 22 Oct 2014

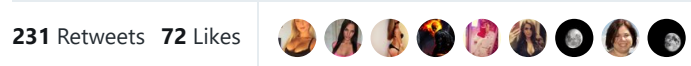

$Q 6 \quad \uparrow\urcorner \quad 231 \quad 72$

dimelo_kan3lla @KandiiKay·22 Oct 2014

Replying to @CTVNews

@CTVNews surprising to learn not all security is armed at the Hill..Y Not ??

๑】

Jen Gehl de Laforest @janiejennifer·22 Oct 2014

Replying to @CTVNews

@CTVNews @ctvsaskatoon so what happened at Rideau? Nothing?

Q

$\uparrow 】$

Global Awareness 101 @Mononoke_Hime ·22 Oct 2014

Replying to @CTVNews

RT @CTVNews Police have clarified that there were two shootings in Ottawa today, not three: at the War Memorial and Parliament Hill. \#Canada

Q

$\uparrow \downarrow$

@Champagneveli $\cdot 22$ Oct 2014

Replying to @CTVNews

“@CTVNews: Police have clarified that there were two shootings in Ottawa today, not three: at the War Memorial and Parliament Hill." wow

๑】

Figure 5.1: An example of a false claim and people's replies to it. From the replies, 231 users chose to trust the claim and re-tweeted it as if it was true, while only 4 users asked for further clarifications.

models adapt to the ambiguity inherent in sentences and obtain more salient information $[150,191]$. Compared to its deterministic counterparts, the stochastic factors incorporated by latent random variables allow multi-modal semantic distributions. Besides, we find that the misinformation datasets in social media are relatively small-sized, only containing thousands or even hundreds of data instances. Deep learning models trained with such datasets are prone to be overfitting. By marginalising over latent variables, a deep latent variable model is more robust against overfitting, which is essential for these small-sized datasets [192]. Therefore, we incorporate a latent distribution to deep learning for the task of claim veracity evaluation with user replies. This work has been accepted as a full paper by the Web Conference 2019 [190]. 


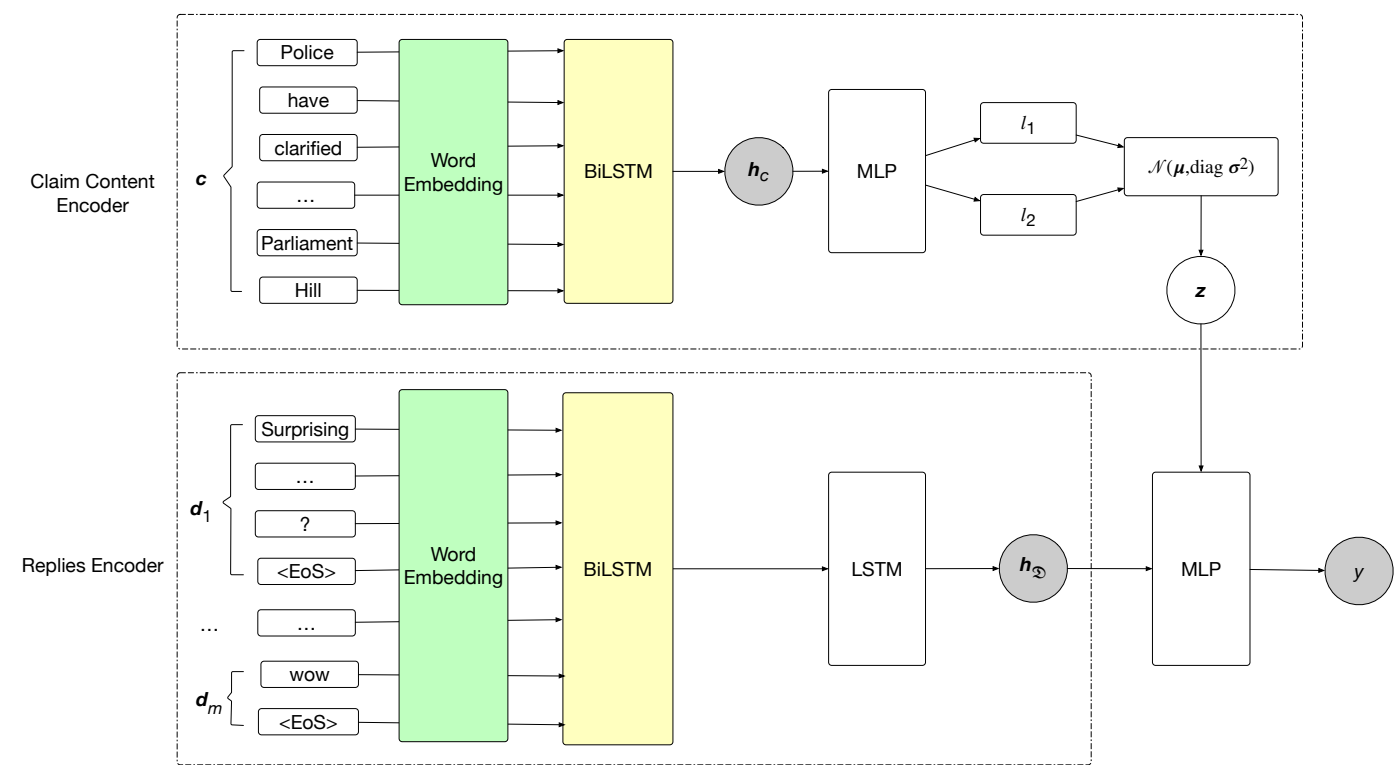

Figure 5.2: The Framework of the deep latent variable model. The framework consists of two major parts, the claim encoder $(\S 5.3 .1)$ and the replies encoder $(\S 5.3 .2)$, the concatenation of which determines the claim veracity. Blocks and nodes represent computation modules and variables. Grey nodes are observed variables while blank nodes are latent variables (similarly with Figure 5.3). Note that blocks of the same color denote the same module.

\subsection{Deep Latent Variable Model}

In this section, we present a deep latent variable model that effectively integrates claim and people's replies. We will first introduce how to encode claim content with deep learning and generate a latent distribution. We then describe the temporalordered approach to encode people's replies, which captures semantic variation along the time line. Finally, we show how to use the latent distribution and people's replies to predict claim veracity. Figure 6.2 describes the proposed deep latent variable model.

\subsubsection{Encoding a Claim}

As content are strong indicators of claim veracity [193], we apply deep learning to extract linguistic features from the claim $c$. To avoid the ambiguity of claims and obtain salient credibility information, we generate a latent distribution based on the extracted linguistic features. Let each claim $c$ be a sequence of discrete words or tokens, i.e., $c=\left[w_{1}, w_{2}, \ldots, w_{l}\right]$, where $w_{l} \in \mathbb{R}^{d}$ is a $d$-dimensional word embedding vector. Based on the sequence of word embeddings, textual features are extracted 
via a Bidirectional Long Short Term Memory (BiLSTM) neural network [194]. The BiLSTM captures long and short semantic dependencies both from previous time steps and future time steps via forward and backward states, and has serves as a de facto basic tool for natural language understanding. The BiLSTM takes as input $c$, converts the sequence of word embeddings into a dense representation, and outputs the concatenation of two hidden states capturing past and future information:

$$
h_{c}=\operatorname{BiLSTM}(c) \text {, }
$$

where $h_{c}$ is the concatenated hidden states from the future and the past directions.

To avoid the ambiguity of claims, instead of a deterministic non-linear transformation, we generate a latent distribution, from which we sample a latent stochastic variable $z$. To embed linguistic information into the latent variable, we set the latent variable to be conditional on $h_{c}$ :

$$
z \sim p_{\theta}\left(z \mid h_{c}\right)
$$

where $p$ is a latent distribution and $\theta$ denotes the non-linear transformation of $h_{c}$ to generate the parameters of $p$. This non-linear transformation is essential to capture higher level representations of $h_{c}$; we implement this non-linear transformation via a Multi-Layer Perceptron (MLP).

We assume that the latent variable $z$ is continuous and follows a multivariate Gaussian distribution. The variable $z$ is parameterized as follows:

$$
p_{\theta}\left(z \mid h_{c}\right)=\mathcal{N}\left(z \mid \mu_{\theta}, \operatorname{diag}\left(\sigma_{\theta}^{2}\right)\right)
$$

where $\mu_{\theta}$ and $\operatorname{diag}\left(\sigma_{\theta}^{2}\right)$ are the mean and the covariance matrix of the multivariate Gaussian distribution. Since the variable $z$ is conditional on the the claim hidden states $h_{c}$, we derive these two parameters of the Gaussian distribution from $h_{c}$ through a deep neural network:

$$
\pi_{\theta}=f_{\theta}\left(h_{c}\right)
$$




$$
\mu_{\theta}=l_{1}\left(\pi_{\theta}\right) \quad \ln \left(\sigma_{\theta}\right)=l_{2}\left(\pi_{\theta}\right),
$$

where $f_{\theta}$ denotes a MLP, $l_{1}$ and $l_{2}$ denote two Linear Transformations (LT). Since LT can generate negative values, to produce $\sigma_{\theta}$ we exponentiate the result of $l_{2}$.

In order to make $\mu_{\theta}$ and $\sigma_{\theta}$ differentiable and backpropagate the loss through the latent distribution $(p)$, the following reparameterization trick is used:

$$
z=\varepsilon \cdot \sigma_{\theta}+\mu_{\theta} \quad \text { where } \varepsilon \sim \mathcal{N}(\mathbf{0}, \mathbf{I})
$$

where $\mathbf{0}$ is a vector of zeros and $\mathbf{I}$ is the identity matrix. By making use of the latent variable $(z)$, our model is able to capture complex noisy patterns in the data.

\subsubsection{Encoding People's Replies}

We now present the people's replies encoder. Replies in social media are chronologically listed as shown in Figure 5.1, where the earliest reply appears at the top of the list. Truth about an event can be gradually manifest as more evidence emerges, thus we assume the latest replies tend to be more reliable and more important than the earlier ones. Based on this assumption, we design a two-layer LSTM to encode replies: the first-layer applies a BiLSTM to summarize the semantic information of each reply and the second-layer applies a LSTM to capture the temporal semantic variation of the replies. Given a claim $c$ commented by a list of replies $\mathfrak{D}=\left\{d_{1}, \ldots\right.$ $\left.\ldots, d_{m}, \ldots, d_{M}\right\}$, these replies are ranked based on their temporal order. The content of a $d_{m}$ consists of a sequence of words $d=\left[w_{1}, \ldots, w_{k}\right]$. To project the claim and replies into the same semantic space, we use the same pre-trained word embeddings for both claims and replies. Hence, $w_{k} \in \mathbb{R}^{d}$ is a $d$-dimensional vector such as the word embedding vector used to encoding the claim. For the sake of semantic coherence, we also employ the same BiLSTM to encode both the claim and its replies. Take the reply $d_{m} \in \mathfrak{D}$ for example, the concatenation of hidden states from forward and backward directions, $h_{d_{m}}$, is denoted as:

$$
h_{d_{m}}=\operatorname{BiLSTM}\left(d_{m}\right)
$$


In order to capture the semantic information of all replies, we sequentially input the concatenated hidden states of each reply into a LSTM. We use the LSTM rather than a BiLSTM because the former gives high weights to recent input, which matches our assumption on the relative importance of the latest reply. Specifically, the LSTM takes the hidden states of each reply as input in a sequential way:

$$
h_{\mathfrak{D}}=\operatorname{LSTM}\left(h_{d}^{M}\right) \text {, }
$$

where $h_{d}^{M}=\left\{h_{d_{1}}, \ldots, h_{d_{m}}, \ldots, h_{d_{M}}\right\}$.

The last hidden state $h_{\mathfrak{D}}$ contains both the linguistic information of each reply and the semantic changes between replies along the time line.

\subsubsection{Veracity Modeling}

In this section we predict veracity by combining the claim and reply information via a MLP. The strong non-linearity of MLPs make them suitable to find complex relationships between the claim and its replies. Specifically, the MLP input is the latent claim variable $z$ concatenated to the hidden state of replies $h_{\mathfrak{D}}$ :

$$
y=\operatorname{MLP}\left(z, h_{\mathfrak{D}}\right) .
$$

This is the final prediction of our model for veracity evaluation.

\subsection{Optimization}

The stochastic variables of our model are non-linear and non-conjugate [195]. Hence, the posterior distribution cannot be derived analytically. To approximate the posterior distribution, we construct an inference model parameterized by $\phi$ to approximate the intractable true posterior $p_{\theta}\left(z \mid h_{c}\right)$; then we derive an objective function to measure how well $p_{\theta}\left(z \mid h_{c}\right)$ is approximated; finally we exploit the Stochastic Gradient Variational Bayes (SGVB) method $[153,196]$ to learn the inference model parameters $\phi$ together with the generative model parameters $\theta$. Figure 5.3 shows the graphical representation of the generative model and the inference model. 


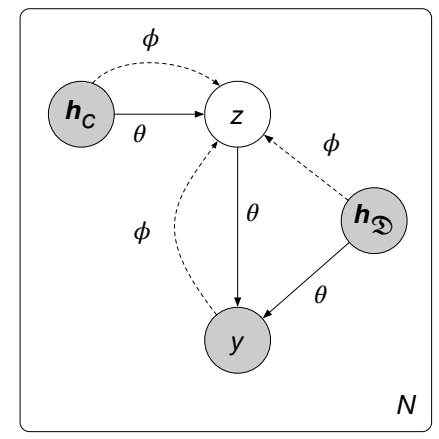

Figure 5.3: The directed graphical model. Grey nodes represent observed variables while blank nodes represent latent variables. Solid lines denote the generative model $p_{\theta}\left(z \mid h_{c}\right) p\left(y \mid z, h_{\mathfrak{D}}\right)$, dashed lines denote the variational approximation $q_{\phi}\left(z \mid y, h_{c}, h_{\mathfrak{D}}\right)$ to the intractable posterior $p_{\theta}\left(z \mid h_{c}\right)$. The variational parameters are learned together with the generative model parameters.

\subsubsection{Inference Model}

Following the neural variational inference approach [153], we construct an inference model (as in Fig. 5.3) parameterized by $\phi$ to compute an approximated posterior distribution, called variational distribution. Given the observed variables, we define a variational distribution $q_{\phi}\left(z \mid y, h_{c}, h_{\mathfrak{D}}\right)$ to approximate the true posterior distribution $p_{\theta}\left(z \mid h_{c}\right)$. Like for the Variational Auto Encoder (VAE) [153], similarly to Eq. 5.3 for $p_{\theta}\left(z \mid h_{c}\right)$, the variational distribution is chosen to be a multivariate Gaussian distribution:

$$
q_{\phi}\left(z \mid y, h_{c}, h_{\mathfrak{D}}\right)=\mathcal{N}\left(z \mid \mu_{\phi}, \operatorname{diag}\left(\sigma_{\phi}^{2}\right)\right)
$$

where $\mu_{\phi}$ and $\operatorname{diag}\left(\sigma_{\phi}^{2}\right)$ are the mean and the covariance matrix of the multivariate Gaussian distribution. We use a deep neural network to derive these two parameters from the observed variables:

$$
\begin{gathered}
\pi_{\phi}=f_{\phi}\left(y, h_{c}, h_{\mathfrak{D}}\right), \\
\mu_{\phi}=l_{3}\left(\pi_{\phi}\right), \quad \ln \left(\sigma_{\phi}\right)=l_{4}\left(\pi_{\phi}\right),
\end{gathered}
$$

where $f_{\phi}$ denotes a MLP, and $l_{3}$ and $l_{4}$ denote two LTs. Note that in the inference model to compute $\mu$ and $\log \sigma$ we use $y, h_{c}, h_{\mathfrak{D}}$ and not only $h_{c}$ as in the generative model. 


\subsubsection{Objective Function}

In the following we derive the objective function of our model following the variational principle. To maximize the $\log$-likelihood $\ln \left(p\left(y \mid h_{c}, h_{\mathfrak{D}}\right)\right)$, we derive an Evidence Lower Bound (ELBO) objective function, which ensures a correct approximation of the true posterior. To simplify the notation of the derivation of the objective function we make the following substitutions: $p_{\theta}(y)=p_{\theta}\left(y \mid z, h_{\mathfrak{D}}\right)$, $p_{\theta}(z)=p_{\theta}\left(z \mid h_{c}\right), q_{\phi}(z)=q_{\phi}\left(z \mid y, h_{c}, h_{\mathfrak{D}}\right)$. The objective function is derived as follows:

$$
\begin{aligned}
\ln \left(p\left(y \mid h_{c}, h_{\mathfrak{D}}\right)\right) & =\ln \left(\int p_{\theta}(y) p_{\theta}(z) d z\right) \\
& \geq \int q_{\phi}(z) \ln \left(\frac{p_{\theta}(z)}{q_{\phi}(z)} p_{\theta}(y)\right) d z \\
& \left.=\mathbb{E}_{q_{\phi}(z)}\left[\ln \left(p_{\theta}(y)\right)\right]-D_{K L}\left[q_{\phi}(z)\right) \| p_{\theta}(z)\right] \\
& =\mathcal{L}\left(\theta, \phi \mid y, h_{c}, h_{\mathfrak{D}}\right),
\end{aligned}
$$

where $\mathcal{L}\left(\theta, \phi \mid y, h_{c}, h_{\mathfrak{D}}\right)$ is the ELBO objective function. The second line of the derivation is possible by using the Jensen's inequality [197]. Since the ELBO objective function is a lower bound of the $\log$-likelihood $\ln \left(p\left(y \mid h_{c}, h_{\mathfrak{D}}\right)\right)$, its maximization maximizes the log-likelihood.

\subsubsection{Gradient Estimation}

Large-scale inference needs minibatch optimization. Thus, we derive a minibatchbased SGVB estimator to differentiate and optimize the ELBO objective function $\left(\mathcal{L}\left(\theta, \phi \mid y, h_{c}, h_{\mathfrak{D}}\right)\right)$ with respect to both the inference parameters $(\phi)$ and the generative parameters $(\theta)$.

Through Monte Carlo estimation we compute the expectation part of the ELBO objective function. Let the minibatch size to be $B$ and, for each claim $c_{i}$ with $i \in$ $[1, B], S$ denotes a sample drawn from the variational posterior distribution $\tilde{z} \sim q_{\phi}$. Given a subset of claims, we can construct an estimator of ELBO objective function 


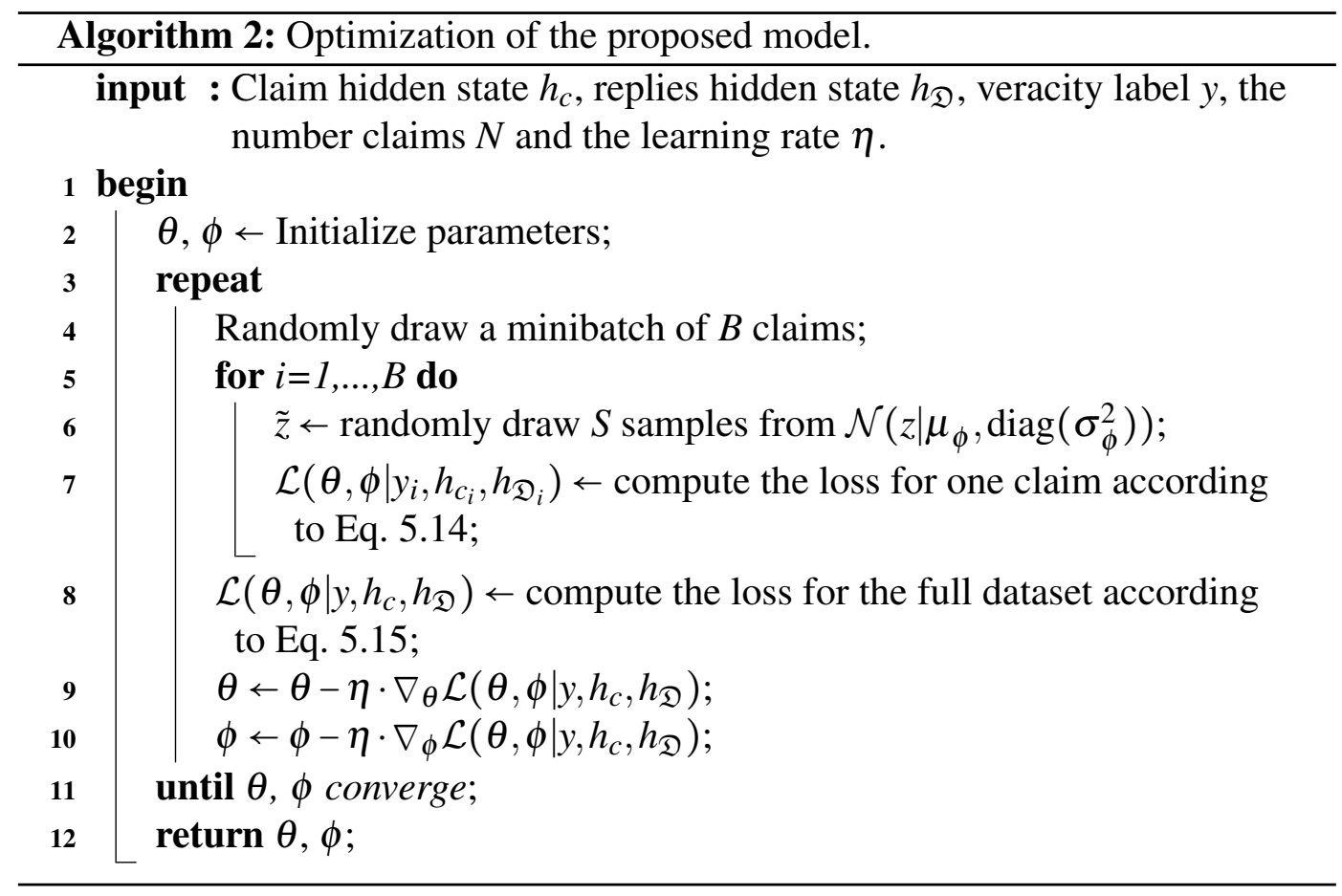

for the full dataset based on mini-batches as follows:

$$
\begin{aligned}
\mathcal{L}\left(\theta, \phi \mid y_{i}, h_{c_{i}}, h_{\mathfrak{D}_{i}}\right)= & \frac{1}{S} \sum_{s=1}^{S}\left[\log p_{\theta}\left(y_{i} \mid \tilde{z}^{(s)}, h_{\mathfrak{D}_{i}}\right)\right] \\
& \left.-D_{K L}\left[q_{\phi}(z)\right) \| p_{\theta}(z)\right] \\
\mathcal{L}\left(\theta, \phi \mid y, h_{c}, h_{\mathfrak{D}}\right) \approx & \frac{N}{B} \sum_{i=1}^{B} \mathcal{L}\left(\theta, \phi \mid y_{i}, h_{c_{i}}, h_{\mathfrak{D}_{i}}\right),
\end{aligned}
$$

where $\mathcal{L}\left(\theta, \phi \mid y_{i}, h_{c_{i}}, h_{\mathfrak{D}_{i}}\right)$ denote the estimates based on the $i$ claim and $N$ is the total number of claims. Algorithm 2 shows the mini-batch gradient descent optimization process for both the generative $(\theta)$ and inference $(\phi)$ parameters. Note that the gradient steps in Algorithm 1 can easily be alternated with a more powerful optimizer such as the Adam algorithm [181].

Although both $q_{\phi}\left(z \mid y, h_{c}, h_{\mathfrak{D}}\right)$ and $p_{\theta}\left(z \mid h_{c}\right)$ are modeled as parameterized Gaussian distributions. The former is an approximation of the latter that only functions during learning. The latter, instead, is the learned distribution from which samples are generated in order to classify claim veracity. 


\subsubsection{Prediction}

After training, we compute the posterior distribution $p_{\theta}\left(z \mid h_{c}\right)$ through the generative network. The actual prediction of a claim veracity is given by taking the expectation of $S$ samples:

$$
\bar{y}=\frac{1}{S} \sum_{s=1}^{S} \operatorname{MLP}\left(z^{s}, h_{\mathfrak{D}}\right),
$$

where $z^{s}$ denote the samples drawn from the true posterior distribution $p_{\theta}\left(z \mid h_{c}\right)$.

\subsection{Experiment Setup}

\subsubsection{Research Questions}

To validate the performance of the proposed model, we seek to answer the following research questions, which will be guide the remainder of the chapter. These ablation studies will help justify the choice of designing such a model.

RQ1 Does our model outperform the state-of-the-art baselines?

RQ2 Does the latent distribution outperforms a deterministic counterpart?

RQ3 Do user replies produce more accurate predictions of claim veracity?

RQ4 Is the temporal order better than random when encoding replies?

RQ5 Is it beneficial to incorporate a latent variable to encode replies?

RQ6 How does the dimension of the latent variable $z$ affect model's performance?

\subsubsection{Datasets}

In order to compare the performance of our proposed model against the baselines, we experimented with two real-world benchmark datasets, the RumourEval [198] and the Pheme [90] datasets. Both datasets contain Twitter conversation threads about claims (like the example shown in Figure 5.1). A conversation thread consists of a tweet making a true or false claim, and branches of people's replies expressing their opinion about it. A summary of the datasets statistics is available in Table 5.1.

The RumourEval dataset has been developed for the SemEval-2017 Task 8 competition. This dataset consists of 325 source tweets and 5,568 user reply tweets. 
Table 5.1: Statistics of the datasets.

\begin{tabular}{l|l|cc|cc}
\hline Subset & Veracity & \multicolumn{2}{|c|}{ RumourEval } & \multicolumn{2}{c}{ Pheme } \\
& & \#Claims & \#Replies & \#Claims & \#Replies \\
\hline \hline Training & True & 83 & 1,949 & 861 & 24,438 \\
& False & 70 & 1,504 & 625 & 17,676 \\
& Total & 153 & 3,453 & 1,468 & 42,114 \\
\hline Validation & True & 10 & 101 & 95 & 1,154 \\
& False & 12 & 141 & 115 & 1,611 \\
& Total & 22 & 242 & 210 & 2,765 \\
\hline Testing & True & 9 & 412 & 198 & 3077 \\
& False & 12 & 437 & 219 & 3,265 \\
& Total & 21 & 849 & 417 & 6,342 \\
\hline
\end{tabular}

The veracity of each tweet can be true (45\%), false (23\%) or unverified (32\%). Since we aim to focus on the difference between true and false claims, we filter out the unverified tweets. The filtered dataset is divided into a training subset, a validation subset and a testing subset. The training subset contains 153 claims with 3,453 replies, the validation subset contains 22 claims with to 242 replies, and the test subset contains 21 claims with 849 replies. Hyper-parameters are tuned on the validation set.

The Pheme dataset is constructed to help understand how users treat online rumour. Like the RumourEval dataset, the Pheme dataset is divided into a training subset, a validation subset and a testing subset. Specifically, $70 \%$ of the claims are randomly selected as training instances, $10 \%$ as validation instances and the rest as testing instances. Users' replies are divided according to the claims. Note that we do not use the leave-one-out evaluation setting that holds all information about testing events from training like the prior work [97]. Instead, we want to simulate the more practical case that there are a certain (or limited) number of annotated claims even for emerging events.

Because these two datasets are relatively small-sized, we repeat the experiments for 10 times and test statistical significance of observed differences between our model and the best baseline, Multitask, using a two-tailed paired t-test. In our experiments, statistical significance is denoted using * for significant differences with $\alpha=0.05$. 


\subsubsection{Evaluation Measures}

The misinformation detection task is a binary classification task. Such tasks are commonly evaluated by the following evaluation measures: Accuracy, $\mathrm{F}_{1}$, Precision, and Recall. Accuracy is a common evaluation measure for classification tasks. However, it is less reliable when datasets suffer from class imbalance. The evaluation measures Precision, Recall and $\mathrm{F}_{1}$ complement Accuracy because not suffering from this problem.

\subsubsection{Hyperparameters Setting}

The activation function of the three LSTMs is tanh. The activation function of the MLPs is ReLu. The hyperparameters tuned on the validation subset are: the dimension of the hidden layer of all three LSTMs is 30; the dimension of the latent variables is 10; the minibatch size is 32; the number of samples used in Monte Carlo estimates is 20 .

State-of-the-art techniques have been employed to optimize the objective function: Dropout [199] is applied to improve neural networks training, L2-norm regularization is imposed on the weights of the neural networks, Adam optimizer [181] is exploited for fast convergence, and stepwise exponential learning rate decay is adopted to anneal the variations of convergence.

\subsubsection{Baselines}

We test our deep latent variable model against six state-of-the-art models. For a fair comparison, only those models using the claim content and users' replies have been selected. We re-train these models and obtain their evaluation performance.

Support Vector Machine (SVM). This model evaluates the performance of manually extracted features. The extracted features from claim content include: bag-of-words representation, presence of URLs, presence of hashtags, proportion of supporting and denying response [198]. These features are then input to a linear Support Vector Machine classifier that achieves the highest misinformation detection performance in the SemEval-2017 Task $8^{1}$;

\footnotetext{
1http://alt.qcri.org/semeval2017/task8/
} 
Convolutional Neural Networks (CNN). This model evaluates the performance of CNNs on the veracity detection task. Apart from the sequential approach such as BiLSTM, the convolutional model is another powerful neural architecture for natural language understanding [57,200-203]. CNN takes as input pre-trained word embeddings generated with Word2Vec [204] trained on the Google News dataset. To capture features similar to $n$-grams, we apply different convolutional window sizes. A max poling layer is applied to compress the output information of the convolutional layers [203];

Tensor Embeddings (TE). This model leverages tensor decomposition to derive concise claim embeddings, which are used to create a claim-by-claim graph on which labels propagate [205];

Evidence-Aware Deep Learning (DeClarE). This model retrieves evidences from replies using claims as a queries [206]. Then both claims and retrieved replies are input into a deep neural network with attention mechanism. Claim veracity is then computed by aggregating over the prediction generated by every claim-retrieved reply pair;

Multitask Learning (Multitask). This model leverages the relationship between two tasks of the veracity detection pipeline [97], rumour stance detection and veracity prediction tasks. The task of rumour stance detection is based on Branch-LSTM, which is not our baseline because it only classify stance but does not predict veracity. The Multitask model is trained on both jointly. We apply the hard parameter sharing mechanism, where different tasks share the same hidden LSTM layers. Task-specific layers takes the shared hidden information and generate per-task predictions;

Tree-structured RNN (TRNN). This model learns discriminative features from replies content by following their non-sequential propagation structure. Among the proposed two structures, we select the top-down structure for tweet representation learning because marginally better than the bottom-up structure [207]. 
Table 5.2: Performance comparison of the proposed deep latent variable model against the baselines.

\begin{tabular}{l|l|c|c|c|c|c|c|c}
\hline Dataset & Measure & SVM & CNN & TE & DeClarE & Multitask & TRNN & Ours \\
\hline \hline RumourEval & Accuracy (\%) & 71.42 & 61.90 & 66.67 & 66.67 & 66.67 & 76.19 & $\mathbf{8 0 . 9 5}^{\star}$ \\
& Precision (\%) & 66.67 & 54.54 & 60.00 & 58.33 & 57.14 & 70.00 & $\mathbf{7 7 . 7 8}^{\star}$ \\
& Recall (\%) & 66.67 & 66.67 & 66.67 & 77.78 & $\mathbf{8 8 . 8 9}$ & 77.78 & $\mathbf{7 7 . 7 8}^{\star}$ \\
& F (\%) & 66.67 & 59.88 & 63.15 & 66.67 & 69.57 & 73.68 & $\mathbf{7 7 . 7 8}^{\star}$ \\
\hline \hline Pheme & Accuracy (\%) & 72.18 & 59.23 & 65.22 & 67.87 & 74.94 & 78.65 & $\mathbf{8 0 . 3 3}^{\star}$ \\
& Precision (\%) & $\mathbf{7 8 . 8 0}$ & 56.14 & 63.05 & 64.68 & 68.77 & 77.11 & $78.29^{\star}$ \\
& Recall (\%) & 75.75 & 64.64 & 64.64 & 71.21 & $\mathbf{8 7 . 8 7}$ & 78.28 & $79.29^{\star}$ \\
& F1 (\%) & 72.10 & 60.09 & 63.83 & 67.89 & 77.15 & 77.69 & $\mathbf{7 8 . 7 8}^{\star}$ \\
\hline
\end{tabular}

\subsection{Results and Discussion}

\subsubsection{Performance Comparison (RQ1)}

Table 5.2 summarizes the classification performance of the baselines and our deep latent variable model on the RumourEval and Pheme datasets. We can observe that: (1) In terms of Accuracy and $F_{1}$, most deep learning-based models, such as ours, TRNN and Multitask, outperform the feature engineering-based models, i.e., SVM. This demonstrates that deep neural networks indeed help to learn better hidden representation of claims and replies. (2) Methods exploring relationships between a claim and its replies, such as ours, TRNN and Multitask, achieve better performance than claim content-based methods like TE and CNN. This demonstrates the significance of utilizing people's replies in the misinformation detection task. (3) Our model significantly outperforms the best baseline on both datasets in terms of the Accuracy and $F_{1}$ metrics, demonstrating its effectiveness in the misinformation detection task. This is true despite, our system is not the best for precision and recall alone. This is because precision and recall alone do not offer a clear picture about the performance since one measure can be increased at the expense of the other.

Specifically, our model achieves the highest accuracy (80.95\%), precision $(77.78 \%)$ and $F_{1}(77.78 \%)$ on the RumourEval test subset, and the highest Accuracy $(80.33 \%)$ and $F_{1}(78.78 \%)$ on the Pheme test subset. Larger is achieved by the PDL on the smaller dataset, which proves that the stochastic approach is more robust than purely deterministic approaches on smaller-scale dataset. The TRNN is the strongest baseline achieving the second highest accuracy and $\mathrm{F}_{1}$ on both RumourEval (76.19\% and $73.68 \%)$ and Pheme (78.65\% and $77.69 \%)$ test subsets. 
Compared to the Multitask baseline [97], our model involves a latent distribution while the model in [97] is deterministic. The basic advantage is that the latent distribution enables to sum over all the possibilities in terms of semantics. From the perspective of optimisation, one of the most important benefits is that marginalising the latent distribution in a Bayesian approach is less prone to overfitting.

Correctly Predicted True Claim

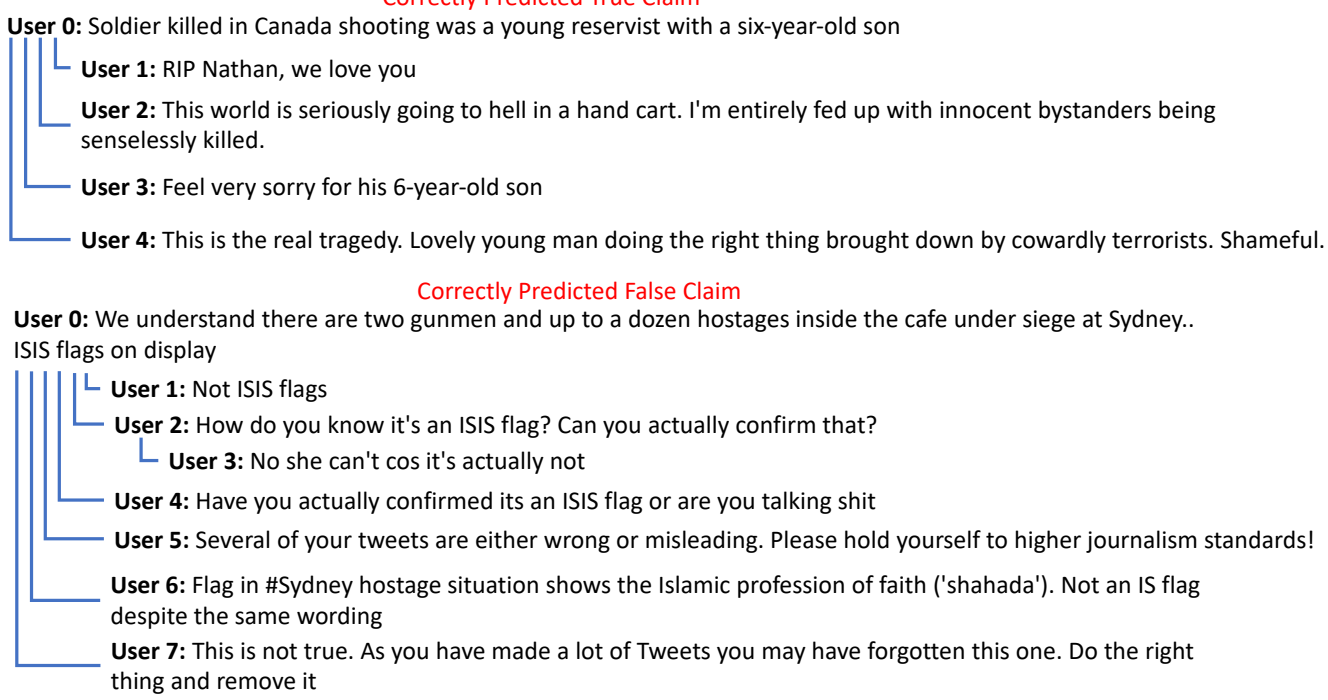

(a) Correctly predicted claims

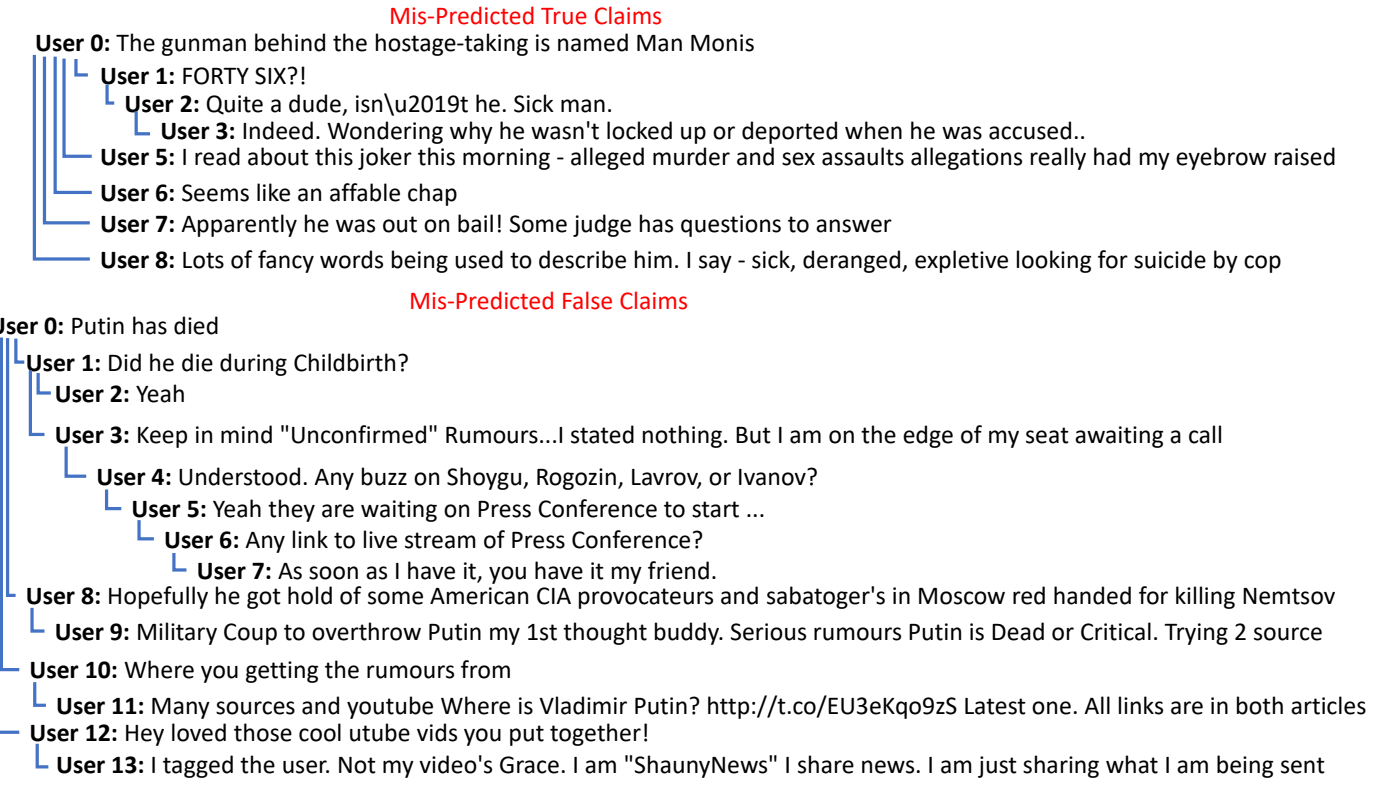

(b) Mis-predicted claims

Figure 5.4: Example data instances from the Pheme dataset that are correctly predicted in Figure (a) and mis-predicted in Figure (b). 
To have a good understanding of the model's predictions, we select four data instances from the pheme dataset, which are correctly predicted true claims, correctly predicted false claims, mis-predicted true claims and mis-predicted false claims. Based on these four instances, we analyse that the proposed model can make correct predictions when user replies are clearly supporting/denying the original claims, or user replies are semantically extension of the claims. However, the model may make mistakes when user replies are not semantically consistent with the claims or there are more semantic information that is out of the claim.

The model is good at processing replies that are flat, that is, replies towards the original claim. Highly hierarchically organized replies can be another error source, as the proposed model deals with replies in a sequence that is chronologically ordered and ignores the branch structure of replies.

\subsubsection{Ablation of the Latent Distribution (RQ2)}

In this subsection, we evaluate the impact of using a latent distribution into the claim encoder on the misinformation detection task. To evaluate the impact of the latent distribution $p$, we ablate $p$ in our model and compare its classification performance against the full model. Specifically, the ablation is done by taking the output of the BiLSTM hidden states, i.e., $h_{c}$ and give this as input to the output MLP. The rest of the model remains unchanged. Since no latent distribution is involved, the ablated model is optimized in accordance with the Softmax loss minimization.

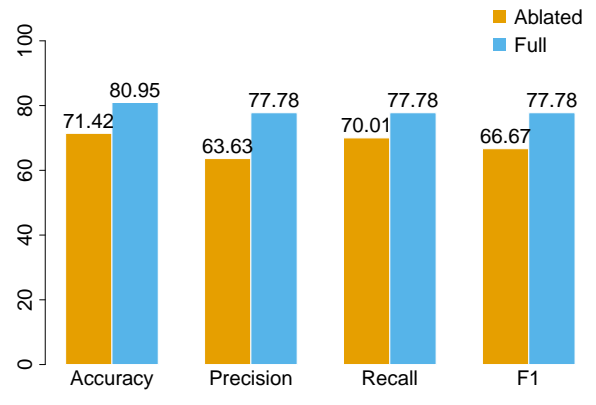

(a) RumourEval

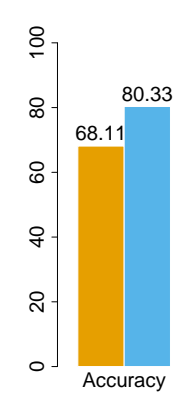

(b) Pheme

Figure 5.5: The impact of the latent distribution $p$ on the model performance. In both figures we show the performance change on all the evaluation measures of the model with (Full) or without (Ablated) $p$. Figure (a) shows it for the RumourEval test subset and Figure (b) shows it for the Pheme test subset. 
In Figure 5.5(a) and 5.5(b) we show the classification performance of the ablated model against the full model on the RumourEval and Pheme test subsets. The full model outperforms the ablated one by at least $7.77 \%$ on every evaluation measure. This demonstrates the effectiveness of introducing stochastic units to claim semantics and credibility information for the veracity evaluation task.

\subsubsection{Ablation of People's Replies (RQ3)}

We now evaluate the contribution people's replies in the misinformation detection task. In order to examine its contribution we compare our full model with and without replies. Specifically, we ablate the input coming from the replies to the final MLP, which now is used only to perform a non-linear transformation of the latent variable $z$.

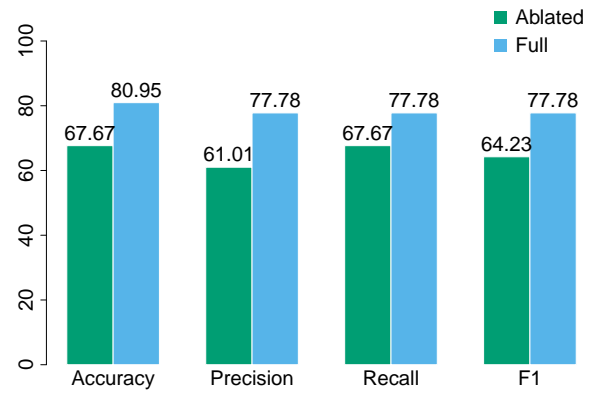

(a) RumourEval

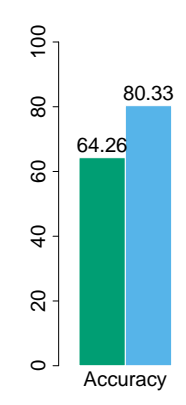

(b) Pheme

Figure 5.6: The impact of people's replies on the model performance. In both figures we show the performance change on all the evaluation measures of the model with (Full) or without (Ablated) replies information. Figure (a) shows performance for the RumourEval test subset Figure (b) shows it for the Pheme test subset.

In Figure 5.6(a) and 5.6(b) we show the classification performance of the ablated model against the full model on the RumourEval and the Pheme test subsets. Here, we observe that the auxiliary information extracted from people's replies has a large impact to the final performance our model. In fact, every evaluation measure is increased by at least $10.11 \%$.

\subsubsection{Random vs. Temporal Ordered Replies (RQ4)}

The proposed model rank people's replies based on the temporal order. In this subsection, we analyze the contribution of ranking the replies according to their 
temporal order. We compare this against a random order.Specifically, we randomize the $h_{d}^{M}$ before it is input to the LSTM.

In Figure 5.7(a) and 5.7(b) we show the performance comparison of replies encoded in these two orders. We observe that the temporal ordered replies achieve better performance than the random ordered. Besides, the random ordered model is still worse TRNN yet better than Multitask. This is probably because TRNN takes the temporal structure of replies into the model while Multitask fail to involve temporal information.

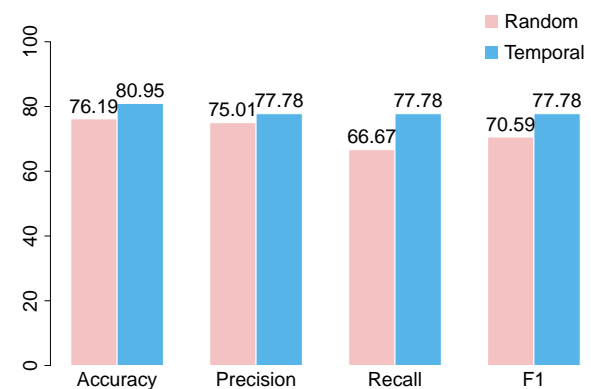

(a) RumourEval

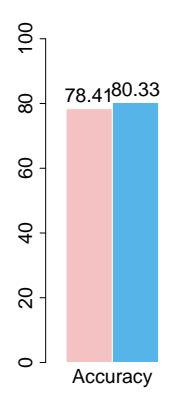

(b) Pheme

Figure 5.7: The effect of the temporal order of the reply encoder on model performance. In both figures we show the performance change on all the evaluation measures of the model with random and temporal ordered people's replies. Figure (a) shows it for the RumourEval test subset and Figure (b) shows it for the Pheme test subset.

\subsubsection{A Latent Distribution for Replies (RQ5)}

Considering the improved performance brought by the latent distribution for claim semantics, in this subsection we answer whether it would be beneficial to incorporate a latent distribution also for semantics of a sequence of user replies. In order to answer this research question, we expand our model by adding a new latent distribution in the reply encoder. Similarly to what done for the claim encoder, the new latent distribution is designed as a multidimensional Gaussian distribution with mean and covariance matrix derived from the LSTM output $h_{\mathcal{D}}$ (as in Eq. 5.3, 5.4 and 5.5). A new latent variable is sampled similarly as in Eq 5.6 and input to the MLP to predicting veracity of the claim. In Figure 5.9(a) and 5.9(b) we show the 


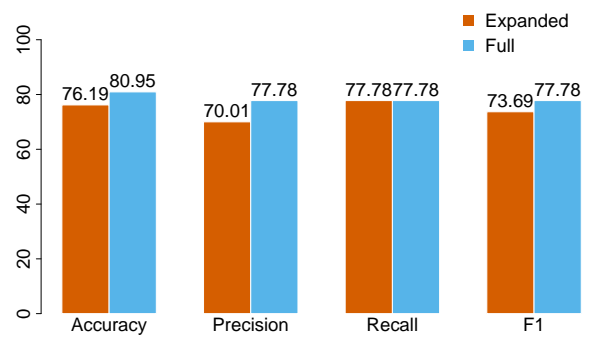

(a) RumourEval
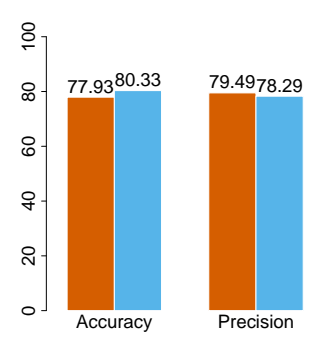

(b) Pheme

Figure 5.8: The effect of an additional latent distribution for people's replies on the model performance. In both figures we show the performance change on all the evaluation measures of the model with (Expanded) and without (Full) an additional latent variable for the people's replies. Figure (a) shows it for the RumourEval test subset and Figure (b) shows it for the Pheme test subset.

model performance comparison. We observe that the new latent distribution does not have an effect on the performance on the model for all the evaluation measures and dataset test subsets. Based on this analysis, we conclude that the incorporation of the additional latent distribution for replies does not provide any additional improvement in performance. This is possibly because that the stochastic factors incorporated by the latent distribution are adequate for claim veracity evaluation. Adding additional randomness for reply ambiguity would make it more difficult for the model to find the proper mapping from claims and replies to veracity predictions. Besides, compared to one latent variable, it is more challenging to approximate the posterior distributions of two latent variables. Based on the finding and analysis, we decide not to add another latent distribution for user replies.

\subsubsection{Sensitivity Analysis (RQ6)}

In this subsection we evaluate the effect of the dimension of the latent variable $z$. To do this after setting a dimension for $z$ we optimize the rest of the hyperparameters on the validation subset. In Figure 5.9(a) and 5.9(b) we show the effect on performance of the dimension of $z$ on both datasets. We observe that the results are similar for both evaluation measures, accuracy and $F_{1}$. Varying the dimension from 1 to 5 the model brings a larger performance improvement than when varying it from 5 to 25. When the dimension is 15 the model obtains the highest accuracy, $81.22 \%$, on the RumourEval test subset while when the dimension is 10 the model obtains the 


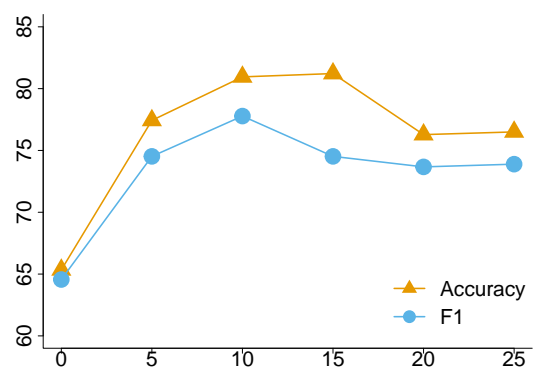

(a) RumourEval

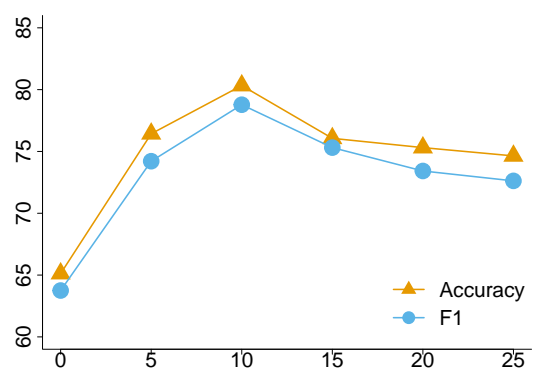

(b) Pheme

Figure 5.9: The effect of the latent variable dimension on model performance. In both figures we show how the accuracy and $\mathrm{F}_{1}$ scores change when varying the dimension of $z$. Figure (a) shows it for the RumourEval test subset and Figure (b) shows it for the Pheme test subset.

highest $\mathrm{F}_{1}, 78.78 \%$, on the RumourEval test subset and highest accuracy, $80.33 \%$ and $\mathrm{F}_{1}, 78.78 \%$, on the Pheme test subset.

These results also show that the increase in model capacity may not necessarily lead to an improvement in performance. The reason could be found on the limited size of the datasets, which might cause overfitting when the model is too complex.

\subsection{Discussion}

\subsubsection{Stance Detection and Veracity Evaluation}

Stance detection has been studied in the context of not only online articles but also user replies in social media, to evaluate claim veracity. Motivated by the task setting of the SemEval-2017 Task 8, Kochkina et al. [97] show that the tasks of stance detection in user replies and rumour verification can be solved by multi-task learning with improved performance. Although our model may indirectly utilize stance expressed in textual replies when determining claim veracity, it is interesting to extend the stance detection model in Chapter 4 from online news articles to social user replies, and more explicitly utilize stance signals to improve veracity evaluation. One challenge of such extension comes from the mismatched stance classes between two scenarios. Stance detection of tweets is differently defined compared to the news stance detection that we study in Chapter 4 . The stance classes of user replies in the SemEval-2017 Task 8 and Pheme datasets are defined to be support- 
ing, denying, commenting and questions [97], and the first three can be mapped to agree, disagree and discuss respectively in news article datasets in Chapter 4. The questioning stance in Pheme and the unrelated stance in news articles characterize the conceptual difference between two scenarios, thus can not be mutually converted. Consequently, there does not exist a semantically hierarchical relationship among these stance classes in Pheme. This absence hinders straightforward application of the hierarchical stance detection architecture to social user replies.

Moreover, it is non-trivial to use the MMD regularization term as a plug-in of alternative stance detection models for user replies. Although it is feasible to manually build a hierarchy among any stance classes, such as questioning versus the other three as the first level and then commenting versus supporting and denying as the second level, MMD may not fit such hierarchy. As we discussed in Chapter 4, agree, disagree and discuss have a concept abstraction- related, which is exactly opposed to unrelated. MMD is designed to disentangle unrelated and the other three stance classes by finding the abstraction. MMD does not hold when the concept abstraction is absent in the manually-built hierarchy. Therefore, it requires more careful design to extend the stance detection model for veracity evaluation.

\subsubsection{Probabilistic Modeling and Uncertainty}

The proposed model involves a latent distribution that incorporates randomness to the mapping from claims and user replies to veracity. The model outputs a probabilistic distribution of veracity predictions, rather than point estimates. This probabilistic manner provides a way of measuring the uncertainty in making predictions. Generally, the uncertainty of deep learning models can be measured in a variety of ways: the entropy of the softmax output, Monte Carlo Dropout in the testing phase [208], a set of predictions from deep ensemble models [209] and a specialised Prior Network [210].

Veracity evaluation is a task where levels of certainty play a crucial role because of the potentially high impact of erroneous decisions. Our probabilistic modeling is rather similar to the deep ensemble model in terms of multiple predictions are made for uncertainty measurement. However, uncertainty measurement is not 
the real probability of a prediction being correct. Instead, it is only a way of ordering predictions from least likely to be correct to mostly likely. Uncertainty measurement can be in a different scale for different datasets and models, thus can be straightforwardly compared. It should be well-calibrated to the actual class membership probability: 0.4 uncertainty means $40 \%$ of predictions are not correct.

Recently, uncertainty measurement of veracity evaluation is studied in [211] to obtain an understanding of a model's decisions. Two types of uncertainty is considered: data uncertainty (aleatoric) due to imperfections in data collection and model uncertainty (epistemic) caused by model parameters. It shows that removing hard instances with high uncertainty filters out many incorrect predictions. Also, claim veracity is a time-sensitive problem: when new information becomes available, the model prediction is supposed to be less uncertain. Therefore, the authors explore an uncertainty dynamics by adding one user reply at a time. This enables to track how uncertainty changes with more user replies.

\subsubsection{Model Deployment}

The proposed deep latent variable model makes use of engaged user replies on social media to infer claim veracity. When deployed on social platforms, there are a few issues to consider. First of all, the model involves $S$ samples from the variational distribution to construct an estimation of the ELBO objective function. The sampling and storage step can be computationally expensive and dependent on the size of $S$. Besides, the model provides a measurement of prediction uncertainty, i.e., the variance of prediction $\bar{y}$. This uncertainty measurement needs to be calibrated in practical applications so as that low uncertainty leads to reliable veracity predictions. When the uncertainty becomes high, the model prediction is less accurate and there tends to be more false predictions. Such calibration process ensures the calculated variance lives to the expectation of practical usage and does not introduce any bias. One further application of the uncertainty measurement is to enable the third veracity class - unverified. It is feasible to produce unverified predictions if the well-calibrated uncertainty is fairly high, in which case the veracity prediction is not confident enough for being either true or false. 


\subsection{Summary}

To predict veracity of claims in social media, we develop a deep learning model with a latent distribution. To enable a deep learning model adapt to semantic ambiguity and obtain salient credibility information, we incorporate the latent distribution whose stochastic factors allow multimodal semantic distribution; and marginalizing the latent distribution makes the model more robust over overfitting in relatively small experimental datasets.

Besides textual replies from engaged users, in the next chapter, we will consider the dynamics of such engagements during misinformation propagation in social media. 


\section{Chapter 6}

\section{Self-Attentive Hawkes Process}

The previous chapters study content-based claim veracity evaluation models that focus on text of claims and engaged user replies. In addition to content-based models, we study a hybrid model that additionally consider how to trace the dynamics of the engagement for tasks such as early detection or spread forecasting. From this chapter on, we pay attention to user engagement dynamics of misinformation in social media. Specifically, users engage in information on social media in the form of a cascade, where the "infection" is initiated by a few nodes that created or posted the false content, and then it interacts with other nodes that get influenced by this piece of misinformation. The engagement process among social users makes a graph, where a new node will be added when an user engages in the information. Such engagements are asynchronous events like commenting and reposting.

We set out to study the temporal evolution of the engagement graph, that is, when and which asynchronous event will be added next. In this chapter, we introduce an attention-based Hawkes Process published in ICML 2020 [212], which works for asynchronous event modeling. In next chapter, we describe how to modify such a Hawkes Process for a hybrid model that considers both content and engagement dynamics.

\subsection{Introduction}

Humans and natural phenomena often generate a large amount of irregular and asynchronous event sequences. These sequences can be, for example, user activ- 
ities on social media platforms [213], high-frequency financial transactions [214], healthcare records [215], gene positions in bioinformatics [216], or earthquakes and aftershocks in geophysics [217]. Three characteristics make these event sequences unique, their: asynchronicity, multi-modality, and cross-correlation. A sequence is asynchronous when multiple events happening in the continuous time domain are sampled with unequal intervals. In contrary to discrete sequences where events have equal sampling intervals. A sequence is multi-modal when sequences contain multiple type of events. A sequence is cross-correlated when the occurrence of one type of event at a certain time can excite or inhibit the happening of future events of the same or another type. Figure 6.1 shows four types of events and their mutual influence. A classic problem with these sequences is to predict which type and when future events will happen.

The occurrence of asynchronous event sequences are often modeled by temporal point processes (TPPs) [154,218]. They are stochastic processes with (marked) events on the continuous time domain. One special but significant type of TPPs is the Hawkes process. A considerable amount of studies have used Hawkes process as a de facto standard tool to model event streams, including: topic modeling and clustering of text document $[219,220]$, construction and inference on network structure [221-223], personalized recommendations based on usersâĂŹ temporal behavior [224], discovering of patterns in social interaction [80,225], and learning causality [161]. Hawkes processes usually model the occurrence probability of an event with a so called intensity function. For those events whose occurrence are influenced by history, the intensity function is specified as history-dependent.

The vanilla Hawkes processes specify a fixed and static intensity function, which limits the capability of capturing complicated dynamics. To improve its capability, recurrent neural networks (RNNs) have been incorporated as result of their success in dealing with sequential data such as speech and language. RNN-based Hawkes processes use a recurrent structure to summarize history events, either in the fashion of discrete-time [226, 227] or continuous-time [165]. This solution brings two benefits: (1) historical contributions are not necessarily additive, and (2) 


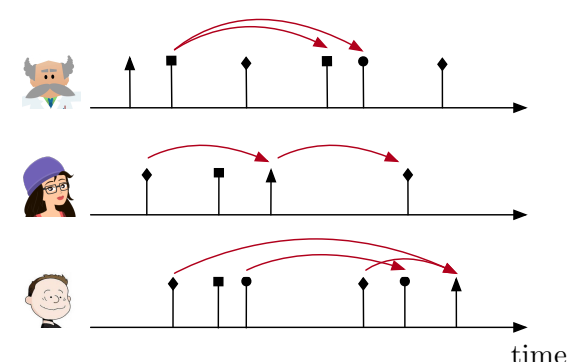

(a)

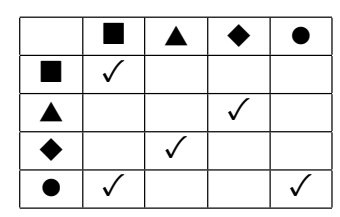

(b)

Figure 6.1: Three users on social media platforms exert different types of actions. The filled dark symbols in (a) mean four action types while the red arrows denote actions influencing another actions. A $\checkmark$ symbol in the cell $(i, j)$ in (b) indicates the influence of the column event type $j$ on the row type $i$ on future events.

allows for complex memory effects such as delay. However, recent developments in natural language processing (NLP) have led to an increasing interest in selfattention. Although self-attention is empirically superior to RNNs in processing word sequences, it has yet to be researched whether self-attention is capable of processing event sequences that are asynchronous, multi-modal and cross-correlated.

In this work we investigate the usefulness of self-attention to Hawkes processes by proposing a Self-Attentive Hawkes Process (SAHP). First, we employ self-attention to measure the influence of historical events to the next event by computing its probability. As self-attention relies on positional embeddings to take into account the order of events, conventional embedding methods are based on sinusoidal functions where each position is distanced by a constant shift of phase, which if used for our sequences would ignore the actual time interval between events. We remedy this deficiency by proposing a time-shifted position embedding method: time intervals act as phase shifts of sinusoidal functions. Second, we argue that the proposed SAHP model is more interpretable than the RNN-based counterparts: The learnt attention weights can reveal contributions of one event type to the happening of another. Code is available at this link ${ }^{1}$. The contributions of this research work can be summarized as follows:

- To the best of our knowledge, this work is the first to link self-attention to Hawkes processes. SAHP inherits improved capability of capturing complicated dynamics and is more interpretable.

\footnotetext{
${ }^{1}$ https://github.com/QiangAIResearcher/sahp_repo
} 
- To consider inter-event time intervals, we propose a time-shifted position embedding that converts time intervals to phase shifts of sinusoidal functions.

- Through extensive experiments on one synthetic dataset and three real-world datasets with different sequence lengths and different numbers of event types, we demonstrate the superiority of SAHP.

\subsection{Related Work}

Neural temporal point process. Complicated dynamics of event occurrence demands for higher capacity of Hawkes processes. To meet this demand, neural networks have been incorporated to modify the intensity function. A discrete-time RNN is proposed in [226] to encode history to fit parameters of the intensity function. A continuous-time Long Short-Term Memory model can avoid encoding time intervals as explicit inputs [165]. Another two works chose not to model the intensity function. The cumulative distribution function is calcculated with a feed forward neural network [228], yet it suffers from two problems: (1) the probability density function is not normalized and (2) negative inter-event times are assigned non-zero probability, as claimed in [229]. Then, it is suggested to model the conditional probability density distribution by a log-normal mixture model [229]. They only studied the one-dimensional distribution of inter-event times, neglecting mutual influence among different event types. Also, despite claimed flexibility, it fails to achieve convincing performance on predicting event types. Above all, history has always been encoded by a recurrent structure.

However, RNN and its variants have been empirically proved to be less competent than self-attention in NLP [6,52]. Moreover, RNN-modified Hawkes processes do not provide a simple way to interpret the peer influence among events. Each historical event updates hidden states in the RNN cells but the process is lack of straightforward interpretability [230,231]. While, our method learns an attention weight tensor, which can quantify the contribution of on event type to another.

Position embedding. Self-attention has to rely on position embeddings to capture sequential orders. [6] computed the absolute position embedding by feeding order 
Table 6.1: The symbols used throughout the paper

\begin{tabular}{ll}
\hline \hline Symbol & Description \\
\hline $\mathcal{U}$ & a set of event types. \\
$\mathcal{S}$ & an event sequence. \\
$u, v$ & the time of an event. \\
$i, j$ & the type of an event. \\
$N_{u}(t)$ & the order number of an event in a sequence. \\
$\mathcal{H}_{t}$ & the counting process for the events of type $u$. \\
$\lambda^{*}(t)$ & the conditional intensity function. \\
$p^{*}(t)$ & the conditional probability density function. \\
$F^{*}(t)$ & the cumulative distribution function. \\
\hline \hline
\end{tabular}

numbers to sinusoidal functions. In contrast, the relative position embeddings use relative distance of the center token to others in the sequence. [232] represented the relative position by learning an embedding matrix. [233] introduced a structural position to model a grammatical structure of a sentence, which involves both the absolute and the relative strategy. However, these methods only consider order numbers of tokens, which ignores time intervals for temporal event sequences.

\subsection{Self-Attentive Hawkes Process}

In this section, we describe how to adapt the self-attention mechanism to Hawkes processes as Figure 6.2 shows.

Event type embedding. The input sequence is made up of events. To obtain a unique dense embedding for each event type, we use a linear embedding layer,

$$
t p_{v}=e_{v} W_{E}
$$

where $t p_{v}$ is the type- $v$ embedding, $e_{v}$ is a one-hot vector of the type- $v$ and $W_{E}$ is the embedding matrix.

Time-shifted positional encoding. Self-attention utilizes positional encoding to inject order information to a sequence. To take into account time intervals of subsequent events, we modify the conventional position encoding. For an event $\left(v_{i}, t_{i}\right)$, the positional encoding is defined as a $K$-dimensional vector such that the $k$-th di- 


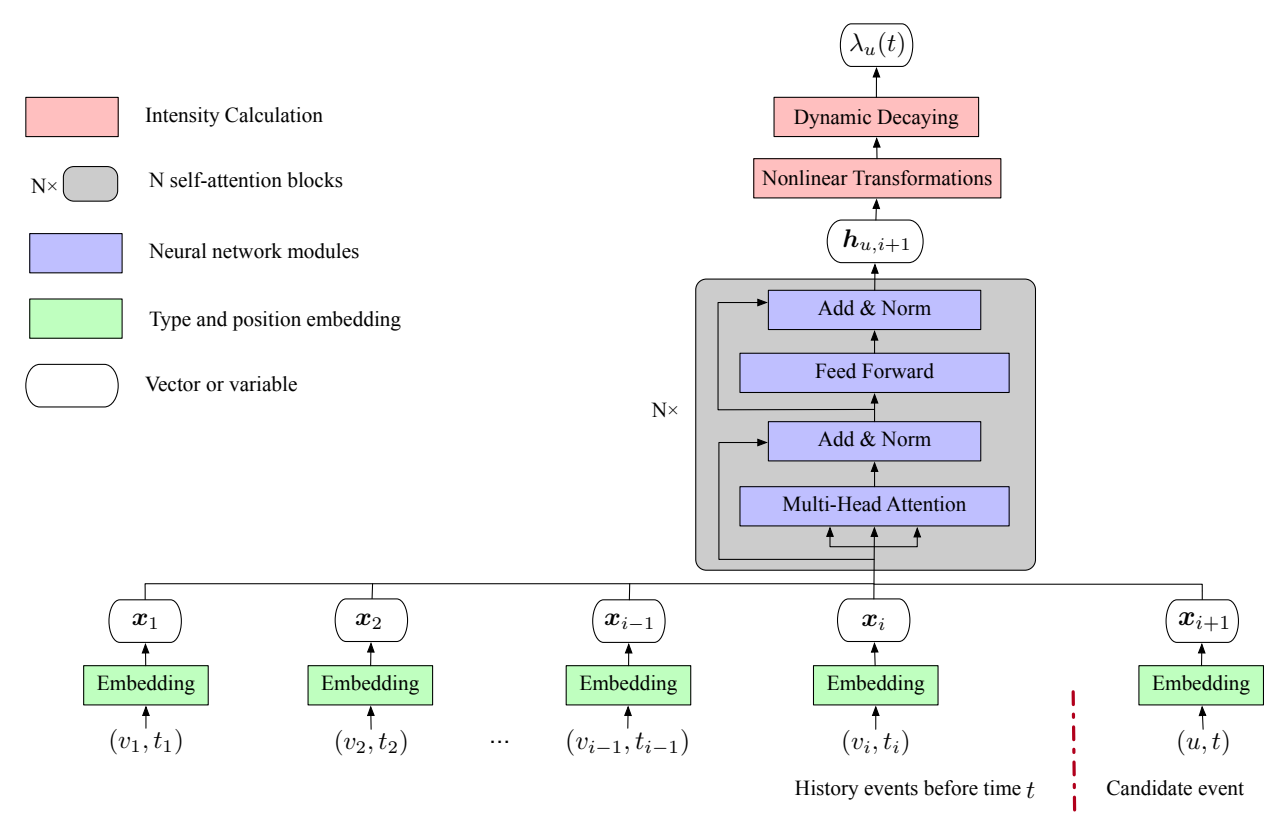

Figure 6.2: An event stream and the SAHP for one event type $(u)$. The intensity function $\lambda_{u}(t)$ is determined by a sequence of past events via the SAHP.

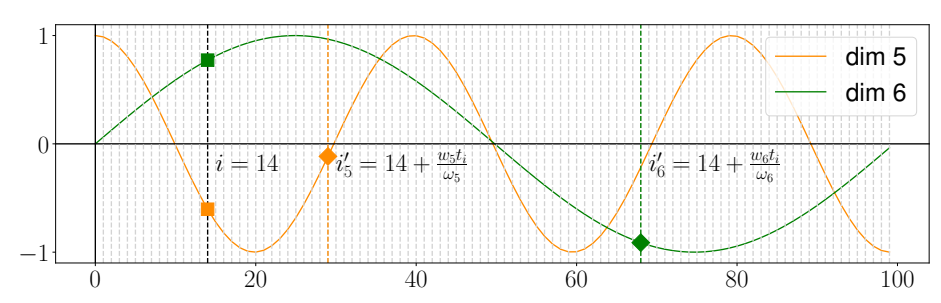

Figure 6.3: The time-shifted position embedding of an event with $i=14$ in a sequence. Squares and diamonds denote conventional and new embedding values with time shifts.

mension of the position embedding is calculated as:

$$
p e_{\left(v_{i}, t_{i}\right)}^{k}=\sin \left(\omega_{k} \times i+w_{k} \times t_{i}\right)
$$

where $i$ is the absolute position of an event in a sequence, and $\omega_{k}$ is the angle frequency of the $k$-th dimension. While $w_{k}$ is a scaling parameter that converts the timestamp $t_{i}$ to a phase shift in the $k$-th dimension. Multiple sinusoidal functions with different $\omega_{k}$ and $w_{k}$ are used to generate the multiple position values, the concatenation of which is the new positional encoding. Even and odd dimensions of pe are generated from sin and cos respectively. 
Figure 6.3 shows how conventional and the new positional encodings work. Suppose an event $\left(v_{i}, t_{i}\right)$ is at the $i=14$ position of a sequence. Conventional methods calculate the values of sinusoidal functions at the $i=14$ position as the position value of this event. Our encoding modifies this by shifting the original position $i$ to a new position $i_{k}^{\prime}=i+\frac{w_{k} t_{i}}{\omega_{k}}$, where $k$ denotes the embedding dimension. This is equivalent to interpolating the time domain and to produce shorter equal-length time periods. Positions in a sequence are thus shifted by the time $t_{i}$. The length of time periods is decided by $\frac{w_{k}}{\omega_{k}}$. Since $w_{k}$ and $\omega_{k}$ are dimension-specific, the shift in one dimension can be different from others.

Historical hidden vector. As an event consists of its type and timestamp, we add the positional encoding to the event type embedding in order to obtain the representation of the event $\left(v_{i}, t_{i}\right)$ :

$$
x_{i}=t p_{v}+p e_{\left(v_{i}, t_{i}\right)}
$$

Self-Attention. Given a series of historical events until $t_{i}$, to compute the intensity of the type- $u$ at the timestamp $t$, we need to consider the influence of all types of events before it. To do this, we compute the pairwise influence of one previous event to the next event by employing self-attention. This generates a hidden vector that summarizes the influence of all previous events:

$$
h_{u, i+1}=\left(\sum_{j=1}^{i} f\left(x_{i+1}, x_{j}\right) g\left(x_{j}\right)\right) / \sum_{j=1}^{i} f\left(x_{i+1}, x_{j}\right),
$$

where $x_{i+1}$ is like query $(q)$ in the attention terminology, $x_{j}$ is the key $(k)$ and $g\left(x_{j}\right)$ is the value $(v)$. The function $g(\cdot)$ is a linear transformation while the similarity function $f(\cdot, \cdot)$ is specified as an embedded Gaussian:

$$
f\left(x_{i+1}, x_{j}\right)=\exp \left(x_{i+1} x_{j}^{T}\right) .
$$

The temporal information is provided to the model during training by preventing the model to learn about future events via masking. We implement this in the attention mechanism by masking out all values in the input sequence that corre- 
spond to future events. Hence, the intensity of one event is obtained only based on its history.

Intensity function. Since the intensity function of Hawkes processes is historydependent, we compute three parameters of the intensity function based on the history hidden vector $h_{u, i+1}$ via the following three non-linear transformations:

$$
\begin{aligned}
& \mu_{u, i+1}=\operatorname{gelu}\left(h_{u, i+1} W_{\mu}\right), \\
& \eta_{u, i+1}=\operatorname{gelu}\left(h_{u, i+1} W_{\eta}\right), \\
& \gamma_{u, i+1}=\operatorname{softplus}\left(h_{u, i+1} W_{\gamma}\right) .
\end{aligned}
$$

The function gelu represents the Gaussian Error Linear Unit for nonlinear activations. We use this activation function because this has been empirically proved to be superior to other activation functions for self-attention [234]. softplus is used for the decaying parameter since $\gamma$ needs to be constrained to strictly positive values.

Finally, we express the intensity function as follows:

$$
\lambda_{u}(t)=\operatorname{softplus}\left(\mu_{u, i+1}+\left(\eta_{u, i+1}-\mu_{u, i+1}\right) \exp \left(-\gamma_{u, i+1}\left(t-t_{i}\right)\right)\right)
$$

for $t \in\left(t_{i}, t_{i+1}\right]$, where the softplus is employed to constrain the intensity function to be positive. The starting intensity at $t=t_{i}$ is $\eta_{u, i+1}$. When $t$ increases from $t_{i}$, the intensity decays exponentially. As $t \rightarrow \infty$, the intensity converges to $\mu_{u, i+1}$. The changing speed is decided by $\left(\eta_{u, i+1}-\mu_{u, i+1}\right)$ that can be both positive and negative. This enables us to capture both excitation and inhibition effects. With inhibition we mean the effect when past events reduce the likelihood of future events [165].

\subsection{Experiments}

\subsubsection{Research Questions}

To examine the model's performance and obtain interpretable results, we seek to answer the following research questions, which will be guide the remainder of the chapter: 
RQ1 How well does our model estimate the ground-truth intensity?

RQ2 What is the overall performance of the proposed model against baselines?

RQ3 How does the number of Monte Carlo samples in the objective function affect model's performance?

RQ4 How efficient the model is in terms of computational time?

RQ5 How can we interpret the SAHP model?

Table 6.2: Statistics of the used datasets.

\begin{tabular}{lrrrrrrr}
\hline \hline Dataset & \# of Types & \multicolumn{3}{c}{ Sequence Length } & \multicolumn{3}{c}{ \# of Sequences } \\
\cline { 2 - 8 } & & Min & Mean & Max & Train & Validation & Test \\
\hline Synthetic & 2 & 68 & 132 & 269 & 3,200 & 400 & 400 \\
RT & 3 & 50 & 109 & 264 & 20,000 & 2,000 & 2,000 \\
SOF & 22 & 41 & 72 & 736 & 4,777 & 530 & 1,326 \\
MMC & 75 & 2 & 4 & 33 & 527 & 58 & 65 \\
\hline \hline
\end{tabular}

To compare our method with the state-of-the-art, we conduct experiments on one synthetic dataset and three real-world datasets. The datasets have been purposefully chosen in order to span over various properties, i.e., the number of event type ranges from 2 to 75 and the average sequence length ranges from 4 to 132. Each dataset is split into a training set, a validation set and a testing set. The validation set is used to tune the hyper-parameters while the testing set is used to measure the model performance. Details about the datasets can be found in Table 6.2. These datasets are all available at the following weblink ${ }^{2}$. Following previous work, we compare models according to testing loss and prediction performance [165, 226]. The evaluation measure used to compare the performance of the models is the loglikelihood (nats) as done in previous work [165].

\subsubsection{Synthetic Dataset}

We generate a synthetic dataset using the open-source Python library tick ${ }^{3}$. A twodimensional Hawkes process is generated with base intensities $\mu_{1}=0.1$ and $\mu_{2}=0.2$.

\footnotetext{
${ }^{2}$ https: / /drive.google.com/drive/folders/0BwqmV0EcoUc8UklIR1BKV25YR1U

${ }^{3}$ https://github.com/X-DataInitiative/tick
} 
The triggering kernels consist of a power law kernel, an exponential kernel, a sum of two exponential kernels, and a sine kernel:

$$
\begin{aligned}
& \phi_{1,1}(t)=0.2 \times(0.5+t)^{-1.3} \\
& \phi_{1,2}(t)=0.03 \times \exp (-0.3 t) \\
& \phi_{2,1}(t)=0.05 \times \exp (-0.2 t)+0.16 \times \exp (-0.8 t) \\
& \phi_{2,2}(t)=\max (0, \sin (t) / 8) \quad \text { for } 0 \leq t \leq 4
\end{aligned}
$$

These four kernels are chosen because they are preliminary and often used to describe how intensity changes as time in the statistical approach to temporal point processes [165]. Simulation is conducted based on the Ogata's algorithm [235]. In Figure 6.4 we show four triggering kernels of the 2-dimensional Hawkes processes. The simulated intensities of each dimension is shown in Figure 6.5.
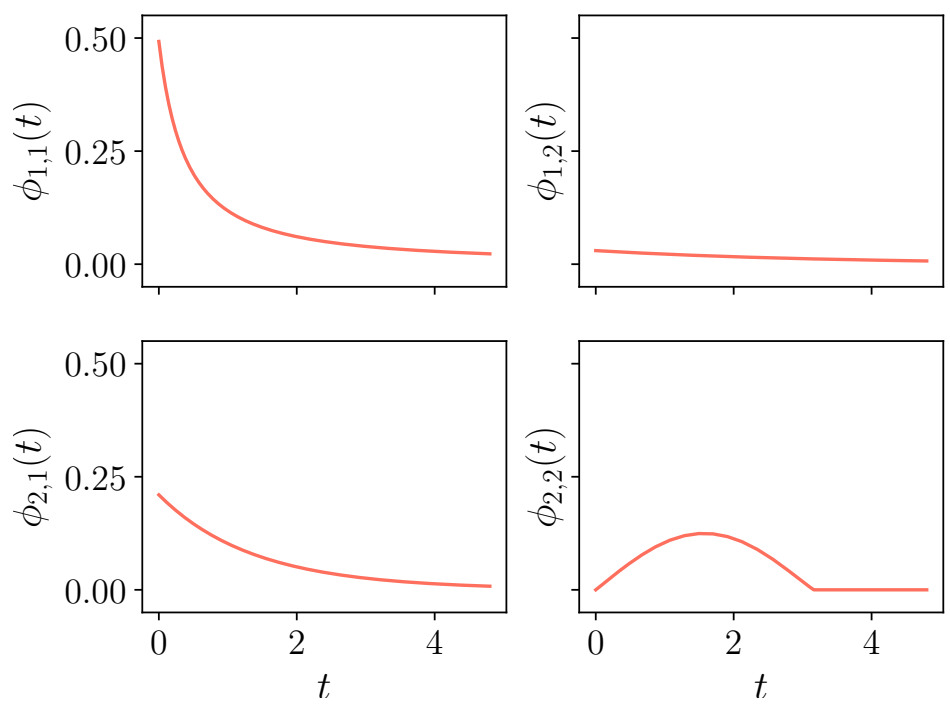

Figure 6.4: The four triggering kernels of the synthetic dataset with 2 event types.

\subsubsection{Real-World Datasets}

In this section, we describe the four datasets that we used to evaluate models' performance on real-world tasks. These datasets are often used by the baselines [165,226] and experimental results are from prior papers of these baselines. 

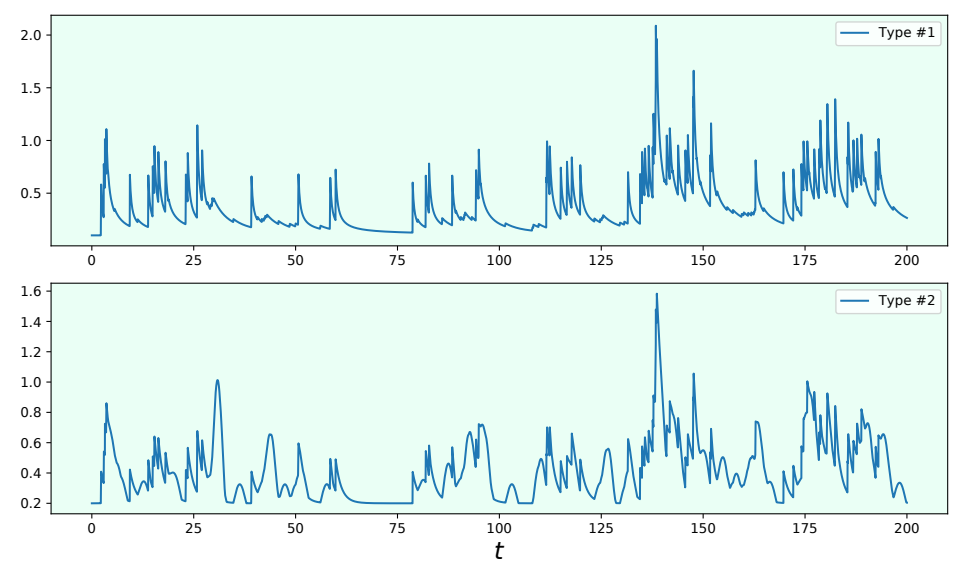

Figure 6.5: The intensities of the two-dimensional Hawkes processes over the synthetic dataset. The upper and the lower subfigure correspond to the dimension- 1 and the dimension-2 respectively.

\subsubsection{Retweet (RT)}

The Retweet dataset contains a total number of 24,000 retweet sequences. In each sequence, an event is a tuple of the tweet type and time. There are $U=3$ types: "small", "medium" and "large" retweeters. The "small" retweeters are those who have fewer than 120 followers, "medium" retweeters have more than 120 but fewer than 1,363 followers, and the rest are "large" retweeters. As for retweet time, the first event in each sequence is labeled with 0 , the next events are labeled with reference to their time interval with respect to the first event in this sequence. The dataset contains information of when a post will be retweeted by which type of users.

\subsubsection{StackOverflow (SOF)}

The StackOverflow dataset has sequences of user awards on StackOverflow where users are awarded based on their proposed questions and their answers to questions proposed by others. There are $U=22$ types of events: Nice Question, Good Answer, Guru, Popular Question, Famous Question, Nice Answer, Good Question, Caucus, Notable Question, Necromancer, Promoter, Yearling, Revival, Enlightened, Great Answer, Populist, Great Question, Constituent, Announcer, Stellar Question, Booster and Publicist. The award time records when a user receives an award. With this dataset, we can learn which type of awards will be given to a user and when. 


\subsubsection{MIMIC-II (MMC)}

The Multiparameter Intelligent Monitoring in Intensive Care (MIMIC-II) dataset is developed based on an electric medical record system. The dataset contains in total 650 sequences, each of which corresponds to an anonymous patient's clinical visits in a seven-year period. Each clinical event records the diagnosis result and the timestamp of that visit. The number of unique diagnosis results is $U=75$. According to the clinical history, a point process captures the dynamics of when a patient will visit doctors and what the diagnose result will be.

\subsubsection{Training Details}

We implement the multi-head attention. This allows the model to jointly attend information from different representation subspaces [6]. The number of heads is a hyper-parameter. We explore this hyper-parameter in the set $\{1,2,4,8,16\}$ and tune it with the validation set. Another hyper-parameter is the number of attention layers. We explore this hyper-parameter in the set $\{2,3,4,5,6\}$. We adapt the Adam as the basic optimizer and develop a warm-up stage for the learning rate whose initialization is set to $1 e-4$. To mitigate overfitting we apply dropout with rate set to 0.1 . $L_{2}$ regularization is used to prevent overfitting and its coefficient in the loss function is $1 e-4$. We train using mini-batches of size 32 over the training sets on an Nvidia GeForce GTX 1080 card. Early stopping is used when the validation loss does not decrease more than $1 e-3$.

\subsubsection{Baselines}

- Hawkes Processes (HP). This is the most conventional Hawkes process statistical model which intensity is described in Eq. 3.6.

- Recurrent Marked Temporal Point Processes (RMTPP). This method [226] uses RNN to learn a representation of influences from past events, and time intervals are encoded as explicit inputs;

- Continuous Time LSTM (CTLSTM). [165] use a continuous-time LSTM, which includes intensity decay and eliminate the need to encode event intervals as numerical inputs of the LSTM. 


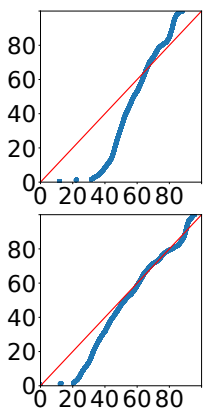

(a) HP

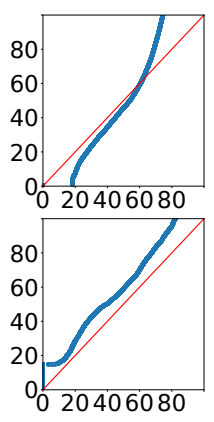

(b) RMTPP

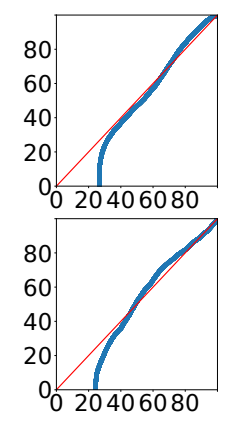

(c) CTLSTM

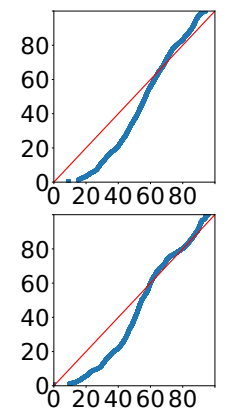

(d) FullyNN

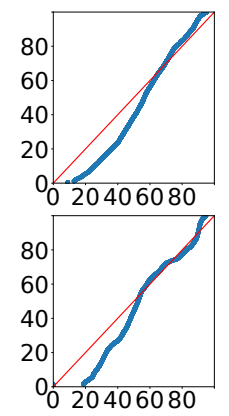

(e) LogNormMix

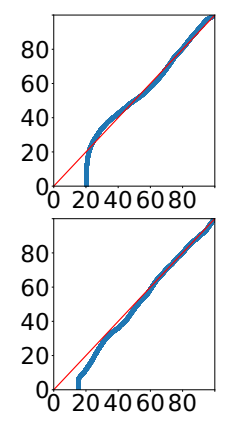

(f) SAHP

Figure 6.6: QQ-plot of true VS estimated intensities of types. The x-axis and the y-axis represent the quantiles of the true and estimated intensities. For each model the top figure is for the type- 1 while the bottom figure is for the type- 2 events.

- Fully Neural Network (FullyNN). [228] propose to model the cumulative distribution function with a feed-forward neural network.

- Log Normal Mixture (LogNormMix). [229] suggest to model the conditional probability density distribution by a log-normal mixture model.

All the used baselines are fitted or trained as indicated in the original papers.

\subsection{Results and Discussion}

For a fair comparison, we tried different hyper-parameter configurations for all models, and selected the configuration with the best validation performance.

\subsubsection{Goodness of fit on the synthetic dataset (RQ1)}

In order to conduct a goodness-of-fit evaluation, we used the synthetic dataset where the true intensity is known, and compared the estimated intensity against the true intensity. We chose the QQ-plot to visualize how well the proposed SAHP is able to approximate the true intensity. Figure 6.6 shows the QQ-plots of intensity estimated by five baselines and SAHP.

From this figure, we observe that the intensity estimated by SAHP produces the most similar distribution to the true one, which indicates that SAHP is able to best capture the underlying complicated dynamics of the synthetic dataset. Moreover, 
by comparing the upper and the lower sub-figures in one column, all models obtain slightly better approximations to the intensity of the second event type.

\subsubsection{Overall Performance (RQ2)}

Sequence modelling Besides evaluation of goodness-of-fit, we further compare the ability of the methods to model an event sequence. As done in previous works [165, 229], negative log-likelihood (NLL) was selected as the evaluation metric. The lower the NLL is, the more capable a model is to model a specific event sequence.

In Table 6.3 we report the per-event NLL of these models on each test set. According to Table 6.3, our method significantly outperforms the baselines in all datasets. As expected, the conventional HP method is the worst in modeling an event sequence in all datasets. RMTPP and CTLSTM have very similar performance except on the Retweet dataset, where CTLSTM achieves a lower NLL than RMTPP.

Table 6.3: Negative log-likelihood per event on the four test sets.

\begin{tabular}{l|rccr}
\hline \hline Dataset & Synthetic & RT & SOF & MMC \\
\hline HP & 2.12 & 9.84 & 3.21 & 1.81 \\
RMTPP & 1.85 & 7.43 & 2.44 & 1.33 \\
CTLMST & 1.83 & 6.95 & 2.38 & 1.36 \\
FullyNN & 1.55 & 6.23 & 2.21 & 1.03 \\
LogNormMix & 1.43 & 5.32 & 2.01 & 0.78 \\
SAHP & $\mathbf{1 . 3 5}$ & $\mathbf{4 . 5 6}$ & $\mathbf{1 . 8 6}$ & $\mathbf{0 . 5 2}$ \\
\hline \hline
\end{tabular}

Event prediction We also evaluate the ability of the methods to predict the next event, including type and the timestamp, according to history. To emphasizes the importance of the time-shifted positional embedding, we also compare SAHP with a version (SAHP-TSE) where the new positional encoding is replaced with the standard one as in [6]. We categorize type prediction as a multi-class classification problem. As there is class imbalance among event types, we use the macro $F_{1}$ as the evaluation metric. Also, since time interval prediction is assumed to be a real number, a common evaluation metric to evaluate these cases is to use the Root Mean Square Error (RMSE). In order to eliminate the effect of the scale of time intervals, 
we compute the prediction error according to

$$
\varepsilon_{i}=\frac{\left(\hat{t}_{i+1}-t_{i}\right)-\left(t_{i+1}-t_{i}\right)}{t_{i+1}-t_{i}}
$$

where $\hat{t}_{i+1}$ is the predicted timestamp while $t_{i+1}$ is the ground truth, and $\hat{t}_{i+1}-t_{i}$ is the predicted time interval while $t_{i+1}-t_{i}$ is the true time interval. The results of type and time prediction are summarized in Table 6.4 and Table 6.5.

These two tables illustrate that our model outperforms the baselines in terms of $F_{1}$ and RMSE on all the prediction tasks. We also observe that SAHP demonstrates a larger margin in type prediction for $F_{1}$. FullyNN and LogNormMix are consistently better than the other baselines in time prediction, yet LogNormMix is not good at predicting event types, which confirms the previous findings [229]. Another important finding is that the use of the time-shifted positional embedding improves the performance of our method in both tasks.

Table 6.4: $F_{1}(\%)$ of event type prediction on the four test-sets.

\begin{tabular}{l|rcrr}
\hline \hline Dataset & Synthetic & RT & SOF & MMC \\
\hline HP & 33.20 & 32.43 & 2.98 & 19.32 \\
RMTPP & 40.32 & 41.22 & 5.44 & 28.76 \\
CTLMST & 43.80 & 39.21 & 4.88 & 34.00 \\
FullyNN & 45.21 & 43.80 & 6.34 & 33.32 \\
LogNormMix & 42.09 & 45.25 & 3.23 & 32.86 \\
SAHP-TSE & 57.93 & 53.24 & 24.05 & 34.23 \\
SAHP & $\mathbf{5 8 . 5 0}$ & $\mathbf{5 3 . 9 2}$ & $\mathbf{2 4 . 1 2}$ & $\mathbf{3 6 . 9 0}$ \\
\hline \hline
\end{tabular}

Table 6.5: RMSE of event timestamp prediction on the four test sets.

\begin{tabular}{l|rccr}
\hline \hline Dataset & Synthetic & RT & SOF & MMC \\
\hline HP & 42.80 & 1293.32 & 221.82 & 7.68 \\
RMTPP & 37.07 & 1276.41 & 207.79 & 6.83 \\
CTLMST & 35.08 & 1255.05 & 194.87 & 6.49 \\
FullyNN & 33.34 & 1104.41 & 173.92 & 5.43 \\
LogNormMix & 32.64 & 1090.45 & 154.13 & 4.12 \\
SAHP-TSE & 33.32 & 1102.34 & 143.54 & 4.03 \\
SAHP & $\mathbf{3 1 . 1 6}$ & $\mathbf{1 0 5 5 . 0 5}$ & $\mathbf{1 3 3 . 6 1}$ & $\mathbf{3 . 8 9}$ \\
\hline \hline
\end{tabular}




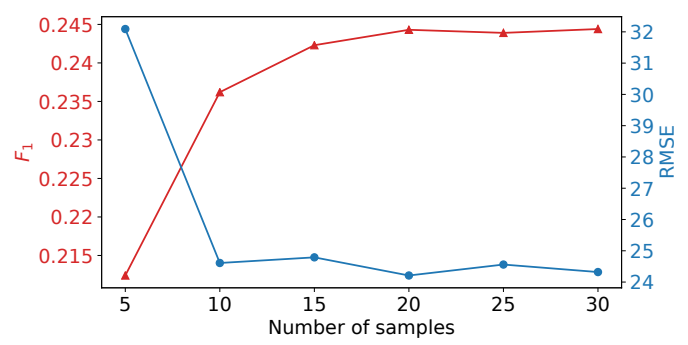

Figure 6.7: The influence of the number of samples of the Monte Carlo estimation on SAHP's performance for event prediction.

\subsubsection{Number of samples' influence (RQ3)}

When we optimize the objective function Eq. 3.8, since it is not a closed form of the expectation, we use Monte Carlo sampling to approximate the integral. This experiment studies how the number of samples influences the SAHP's performance. The number of samples varies from 5 to 30 with step size 5 . We report experimental results obtained from the StackOverflow dataset; other datasets share similar findings.

Figure 6.7 describes how the performance of event prediction changes with different number of samples. From 5 to 10 samples, there is a significant improvement on the evaluation metrics. With more than 10 samples, we observe that the performance plateaus. To reduce computational time, we use 10 as the default number of samples in Monte Carlo.

\subsubsection{Computational Efficiency (RQ4)}

To compare the computational efficiency of our method with neural baselines, we report in Table 6.6 the running time on the retweet dataset with a Titan Xp GPU card. The mini-batch size is 32 and running time is averaged by 10 epochs. We make two observations: (1) CTLSTM is the least computationally efficient model, which could be due to the recurrent architecture and Monte Carlo sampling, and (2) the proposed SAHP model enjoys the same level of computational efficiency with LogNormMix and FullyNN.

\subsubsection{Model interpretability (RQ5)}

Apart from strong capacity in reconstructing the intensity function, the other advantage of our method is its higher interpretability. SAHP is able to reveal peer 
Table 6.6: Model running time on the retweet dataset with a Titan Xp GPU card.

\begin{tabular}{l|r}
\hline \hline Model & Time (Second) \\
\hline RMTPP & 92.22 \\
CTLSTM & 134.69 \\
LogNormMix & 85.32 \\
FullyNN & 87.53 \\
SAHP & 86.97 \\
\hline \hline
\end{tabular}

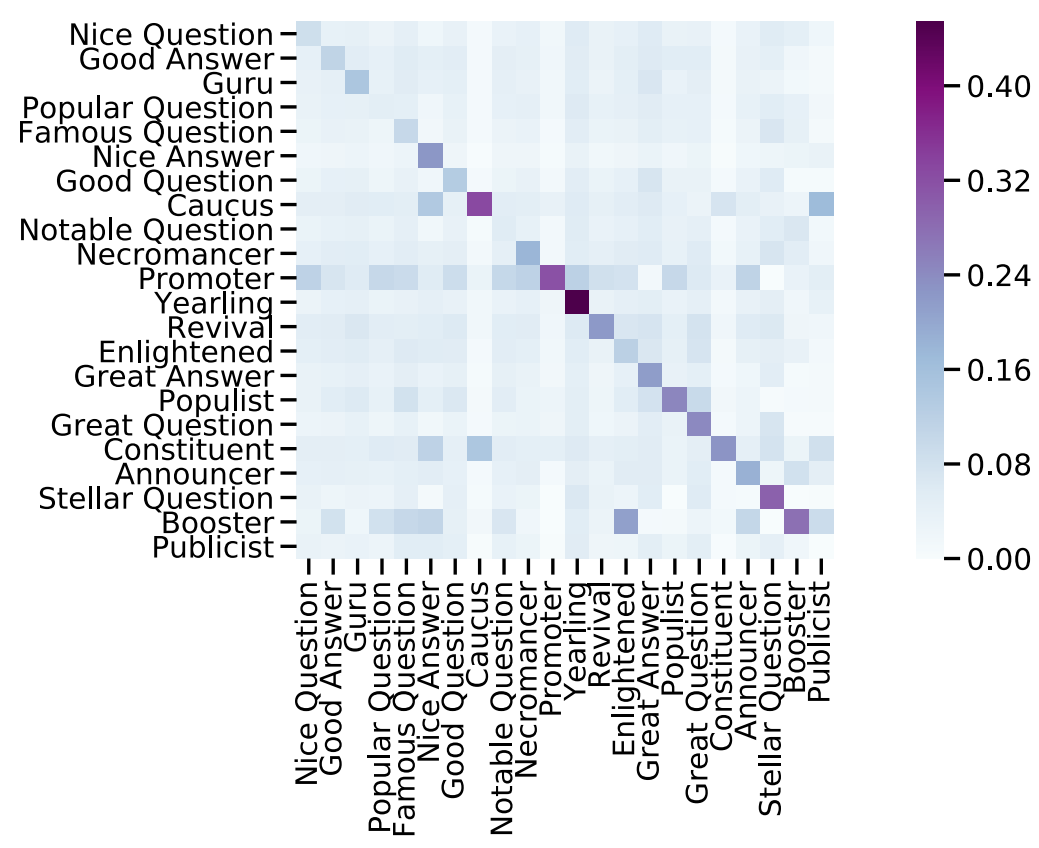

Figure 6.8: Expected attention weights among event types on the StackOverflow test set.

influence among event types. To demonstrate that, we extract the attention weight that the type- $u$ events allocate to the type- $v$ events and accumulate such attention weight over all the sequences on the StackOverflow test set. We remove the effect of the frequency of the $(u, v)$ pairs in the dataset through dividing the accumulated attention weight via the $(u, v)$ frequency. After normalization, we obtain the statistical attention distribution as shown in Figure 6.8. The cell at the $u$-th row and $v$-th column means the statistical attention that the type- $u$ allocates to the type- $v$.

Two interesting findings can be drawn from this figure: 1) for most cells in the diagonal line, when the model computes the intensity of one type, it attends to the history events of the same type; 2) for dark cells in the non-diagonal line, such as (Constituent, Caucus), (Boosters and Enlightened) and (Caucus and Pub- 
licist), the model attends to the latter when computing the likelihood of the former. The first finding is attributed to the fact that attention is computed based on similarity between two embeddings while the second finding indicates the statistical co-occurrence of event types in a sequence.

\subsection{Summary}

In this chapter, we propose a self-attentive Hawkes process where self-attention is adapted to enhance the expressivity of Hawkes processes. This method enhances the model prediction and model interpretability. For the former, the proposed method outperforms state-of-the-art methods via better capturing event dependencies; while for the latter, the model is able to reveal peer influence via attention weights.

For the next chapter, we will leverage this work to model the asynchronous user engagements in misinformation propagation on social media platforms. 


\section{Chapter 7}

\section{Engagement Dynamics via Hawkes}

\section{Processes}

To expand the content-based models for claim veracity evaluation, this chapter presents a hybrid model that utilizes the textual content and dynamics of user engagements in misinformation on social media platforms. Our principal goal is to utilise the maximum space of these engagement patterns, so as to make the most effective use of the available data. Specifically, we first formulate the user engagement in misinformation as a dynamic graph, then employ a modified temporal point process model, i.e., Self-Attentive Hawkes Process presented in Chapter 6, to extract the temporal evolution and the geometric pattern of the dynamic graph. Further, we use it to model the growth of the temporal network scale, so as to forecast the future rate of spread for a claim identified as misinformation. This work has been accepted as a short paper by ECIR 2021 [236].

\subsection{Problem Formulation}

This section gives definitions and describes symbols used in this chapter. A source claim takes the form of $c=(x, t)$, where $x$ is a concatenation of the posting user account features and the claim's text features, i.e. $x=[u \| M]$. Here, $u$ is the user account representation and $M$ is the text message representation. $t$ is initially zero, as ensuing engagement events are timestamped with respect to the source claim, measured in minutes. 
Suppose the claim $c$ is accompanied by a sequence of social engagements $\mathcal{S}=\left\{v_{1}, v_{2}, \ldots, v_{j}, \ldots, v_{N}\right\}$, where $v_{j}=\left(x_{j}, t_{j}\right)$. Similarly, $x_{j}$ is the feature of an engaging node and $t_{j}$ is the engagement time with respect to claim post time. Social engagements include all forms of interactions that users conduct with claims on social media platforms, such as reposting, commenting and tagging.

Static Engagement Graph. Existing works use the claim and its social interactions to form a tree-shaped engagement graph. Here we will a definitive description. Let $\mathcal{G}=<\mathcal{V}, \mathcal{E}>$ denote an engagement graph [237, 238], where $\mathcal{V}$ is defined as $\mathcal{V}=\left\{c, v_{1}, v_{2}, \ldots, v_{j}, \ldots, v_{N}\right\}$. Meanwhile $\mathcal{E}$ is defined as $\mathcal{E}=\left\{e_{i, j}\right\}$ representing a set of directed edges from a source node $v_{i} \in \mathcal{V}$ to a destination node $v_{j} \in \mathcal{V}$. The neighbourhood of node $v_{i}$ is denoted by $\mathcal{N}_{i}=\left\{v_{j}: e_{i, j} \in \mathcal{E}\right\}$. Here we assume $0 \leq i \leq N$ and $0 \leq j \leq N$. Let $A_{i}$ be the corresponding adjacency matrix of $\mathcal{G}_{i}$.

Dynamic Engagement Graph. Static engagement graphs ignore temporal information that is inherent to the dissemination process. To overcome this, we define a dynamic engagement graph. Our temporal, dynamic graph is represented as a sequence of time-stamped snapshots $\mathcal{G}=\left\{\mathcal{G}\left(t_{0}\right), \mathcal{G}\left(t_{1}\right), \cdots, \mathcal{G}\left(t_{j}\right), \cdots, \mathcal{G}\left(t_{N}\right)\right\}$, where the first snapshot simply represents the source claim node and further snapshots are added with each representing the state of the dissemination network when a new node is connected. Let $\mathcal{G}(t)=<\mathcal{V}(t), \mathcal{E}(t)>$ denote the state of the temporal graph $\mathcal{G}$ at time $t$, where $\mathcal{V}(t)=\left\{c, v_{1}, v_{2}, \ldots, v_{j}, \ldots, v_{N(t)}\right\}$, with $N(t)$ being the number of nodes to have directly or indirectly interacted with the claim $c$ as of time $t$. Such a snapshot is defined in the same manner as the previously articulated static engagement graph. A new graph snapshot $\mathcal{G}\left(t_{j+1}\right)$ is generated when a node $v_{j+1}$ is added to the sequence of social engagements. The graph structure of an exemplary false claim's dissemination tree is demonstrated in Figure 7.2.

Task Formulation. With a claim and its social engagements, i.e., $\{c, \mathcal{S}\}$, we form a graph $\mathcal{G}$ and an event sequence $\mathcal{X}$ to conduct the two-part task: (1) predicting the timestamp of the next engagement based on previous engagements, (2) forecasting the rate of change in the number of edges defining the dynamic graph. Notations used in this paper are summarized in Table 7.1. 


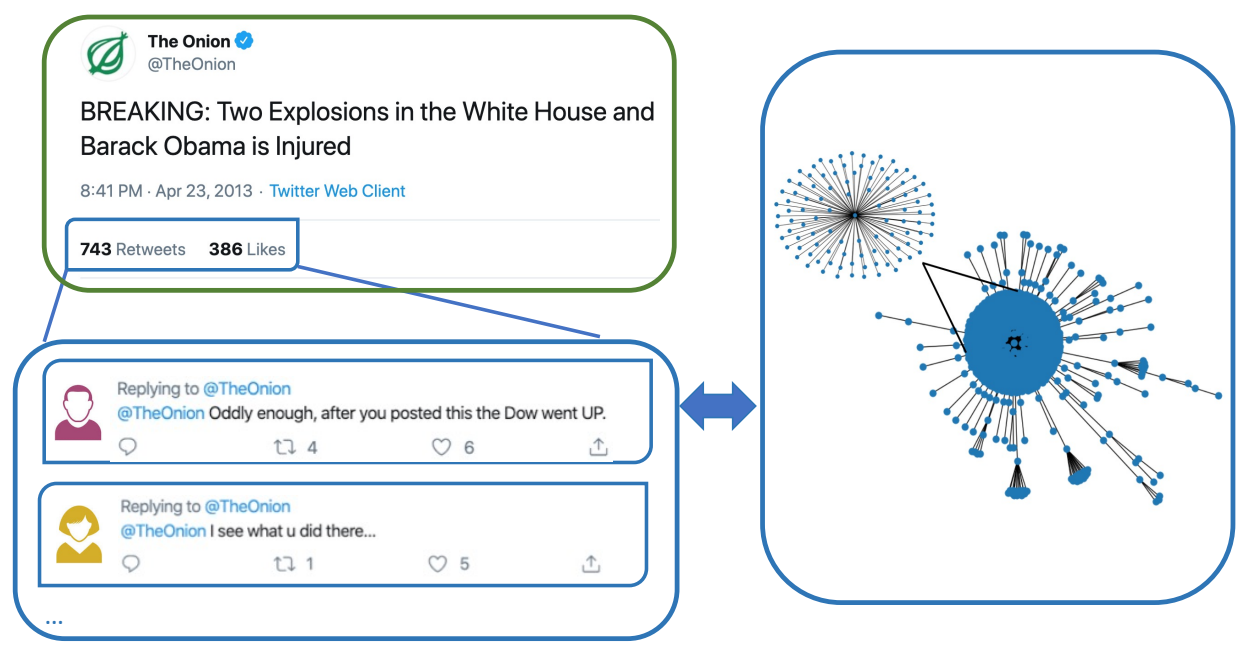

Figure 7.1: Graph representation of source claim dissemination tree, where nodes represent interaction events such as comments and retweets.

Table 7.1: Description of the notations used in this paper.

\begin{tabular}{ll}
\hline \hline Notations & Descriptions \\
\hline$c$ & the claim being verified \\
$x$ & node features \\
$u$ & a user account representation \\
$m$ & a text message representation \\
$t$ & a timestamp \\
$v$ & a social engagement \\
$\mathcal{S}$ & a sequence of social engagements for a claim \\
$\mathcal{G}$ & an engagement graph on social platforms \\
$A$ & an adjacency matrix \\
$i, j$ & the index of social engagements in a sequence, \\
$N$ & also the index of nodes in a graph \\
$y$ & the total number of social engagements for a claim \\
$\mathbb{Y}$ & the veracity of a claim being verified \\
\hline \hline
\end{tabular}

Graph neural networks can extract geometric engagement patterns. Graph Convolutional Networks (GCN) is used in [237] to encapsulate the engagement structure of heterogeneous data. Graph-Aware Co-Attention Network (GCAN) is proposed in $[23,238]$ to utilise the co-attention mechanism in graph modeling. All these works use static graphs and researchers neglect temporal information. 

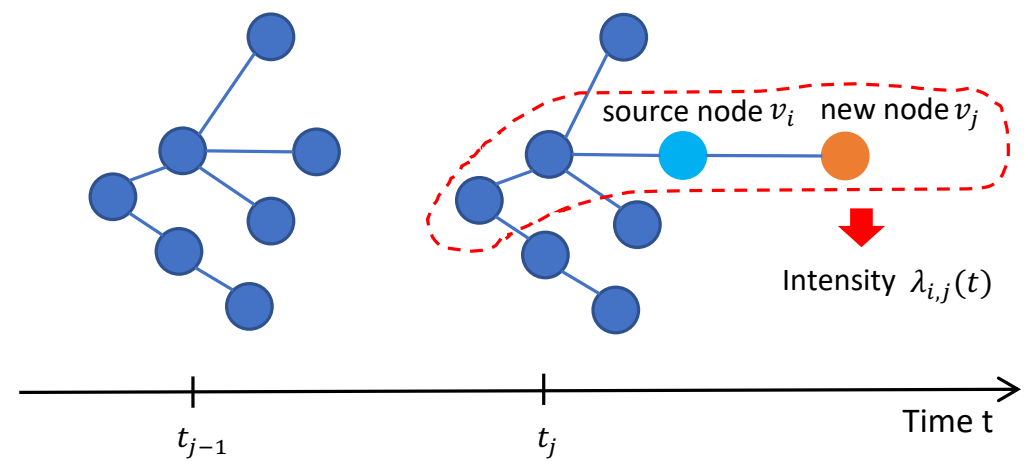

Figure 7.2: An illustrative scheme of the engagement graph by temporal point processes.

\subsection{Hybrid Model Description}

With the temporal evolution of the engagement graph $\mathcal{G}(t)$, new engagement nodes will establish edges with existing nodes and thus update the graph. To capture both geometric and temporal engagement features, we view the addition of new engagement nodes as the chronological events and modify SAHP to generate node embeddings of the dynamic graph $\mathcal{G}(t)$.

\subsubsection{Engagement by Temporal Point Processes}

In our engagement graph use-case, the timestamped event sequence comprises static graph snapshots. This static engagement graph represents the final state of the misinformation dissemination tree. Symbolically, $\mathcal{S}=\left\{\left(x_{j}, t_{j}\right)\right\}_{j=1}^{N}$, where $x_{j}$ are the event features (previously node features) and $t_{j}$ is the timestamp of the $j^{\text {th }}$ event in the sequence $\mathcal{S}$. Intuitively, the added edge $e_{i, j}$ between the source node $v_{i}$ and the new node $v_{j}$ are influenced by not only $v_{i}$ and $v_{j}$, but also the history nodes of $v_{i}$. With this assumption, we define the intensity function associated with adding the new edge $e_{i, j}$ as,

$$
\lambda_{i, j}(t)=g\left(x_{i}, x_{j}\right)+\sum_{i^{\prime} \in \mathcal{H}^{i}} \alpha_{i^{\prime} j}(t) f\left(x_{i^{\prime}}, x_{j}\right) \kappa\left(t-t_{i^{\prime}}\right) .
$$

where $\mathcal{H}^{i}$ contains history events of the node $i$. The function $g(\cdot)$ calculates the affinity between two nodes, which is implemented as a bilinear interaction with the trainable parameter $\mathbf{W}_{1}$, i.e., $f\left(x_{i}, x_{j}\right)=x_{i} * \mathbf{W}_{1} * x_{j}$. A non-linear activation ReLU is used to define the base intensity $g(\cdot)=\operatorname{ReLU}(f(\cdot))$. For history nodes before time 
$t$, we calculate attention weight for each node,

$$
\alpha_{i^{\prime} j}=\frac{\exp \left(f\left(x_{i^{\prime}}, x_{j}\right)\right)}{\sum_{k \in \mathcal{H}^{i}} \exp \left(f\left(x_{k}, x_{j}\right)\right)} .
$$

With the intensity function, we derive the probability of having a new node $v_{j}$ following an existing node $v_{i}$ at the timestamp $t$,

$$
p\left(v_{i}, v_{j} \mid \mathcal{H}^{i}(t)\right)=\frac{\lambda_{i, j}(t)}{\sum_{i^{\prime} \in \mathcal{H}^{i}(t)} \lambda_{i^{\prime}, j}(t)} .
$$

The objective function to minimize is the negative log-likelihood of all the events in the sequence,

$$
\mathcal{L}_{T P P}=-\sum_{t \in \mathcal{T}} \sum_{\left(v_{i}, v_{j}, t\right) \in \mathcal{E}} \log p\left(v_{i}, v_{j} \mid \mathcal{H}^{i}(t)\right)
$$

Negative sampling is used to generate non-existing edges in the objective function as done in [239], so that the learnt node embeddings are able to distinguish which two nodes are connected and which two are not, i.e., the geometric structure. Maximizing the intensity at occurrence timestamps while minimizing the intensity otherwise will enforce the node embeddings to capture temporal dynamics.

\subsubsection{Predictive Task}

Temporal Point Processes allows to give a precise indication of when the next engagement event will occur. With the intensity function, the time density of the subsequent event is calculated as:

$$
p_{i+1}(t)=P\left(t_{i+1}=t \mid \mathcal{H}\left(t_{i+1}\right)\right)=\lambda(t) \exp \left(-\int_{t_{i}}^{t} \lambda(s) d s\right)
$$

where $\lambda(t)=\sum_{u} \lambda_{u}(t)$. The prediction of the next event timestamp $t_{i+1}$ is equal to the following expectation:

$$
\hat{t}_{i+1}=\mathbb{E}\left[t_{i+1} \mid \mathcal{H}\left(t_{i+1}\right)\right]=\int_{t_{i}}^{\infty} t \times p_{t_{i+1}}(t) d t
$$


Because this integral is not solvable analytically we approximate it via Monte Carlo sampling. By accumulating the predicted engagements, we are able to quantitatively describe the evolution pattern of the network scale.

Since time interval prediction is assumed to be a real number, a common evaluation metric to evaluate these cases is to use the Root Mean Square Error (RMSE). In order to eliminate the effect of the scale of time intervals, we compute the prediction error according to the formula as follows $\varepsilon_{i+1}=\frac{\left(\hat{t}_{i+1}-t_{i}\right)-\left(t_{i+1}-t_{i}\right)}{t_{i+1}-t_{i}}$, where $t_{i+1}$ is the ground truth, and $\hat{t}_{i+1}-t_{i}$ is the predicted time interval while $t_{i+1}-t_{i}$ is the true time interval. Predictive loss is measured by $\mathcal{L}_{\text {Pred }}=\sum_{i} \varepsilon_{i+1}$.

\subsubsection{Veracity Classification}

We have designed a temporal point process to capture the geometric structure and temporal evolution of the engagement graph. With node embeddings, we obtain the graph embedding by concatenating the mean pooling and the maximum pooling of all nodes as well as the source claim being verified,

$$
x_{G}=[\operatorname{MeanPool}(\mathcal{S})\|\operatorname{MaxPool}(\mathcal{S})\| c]
$$

Veracity prediction is through a Multi-Layer Perceptron (MLP)

$$
\hat{\mathbf{y}}=\operatorname{softmax}\left(\operatorname{ReLU}\left(\mathbf{W}_{2} x_{G}+\mathbf{b}\right)\right)
$$

where $\mathbf{W}_{2}$ and $\mathbf{b}$ are trainable parameters. The classification loss is calculated by cross-entropy:

$$
\mathcal{L}_{M L P}=-y \log \left(\hat{y}_{1}\right)-(1-y) \log \left(1-\hat{y}_{0}\right) .
$$

We take the weighted sum of the TPP loss, predictive loss and the MLP loss as the final loss function

$$
\mathcal{L}=\mathcal{L}_{T P P}+\omega_{1} * \mathcal{L}_{\text {Pred }}+\omega_{2} * \mathcal{L}_{M L P}
$$


Table 7.2: Statistics of the Twitter 15 and Twitter16.

\begin{tabular}{lrr}
\hline \hline & Twitter15 & Twitter16 \\
\hline \# Source Tweets & 742 & 412 \\
\# True & 372 & 205 \\
\# False & 370 & 207 \\
\# Users & 190,868 & 115,036 \\
avg. retweet per story & 292.19 & 208.70 \\
\hline \hline
\end{tabular}

\subsection{Experiments}

We seek to answer the following research questions in terms of the model performance on detecting misinformation and predicting the dynamics:

RQ1 How is the performance of the model for misinformation detection?

RQ2 How good our model is at predicting the timestamp of the next engagement based on previous engagements?

We use three Twitter datasets [78], i.e., Twitter15 and Twitter16, as well as the Pheme dataset detailed in Chapter 2. Each dataset has a collection of stories with a source tweet being verified and a sequence of its retweets. We pick "True" and "False" source tweets, and split the dataset into training, validation and test sets with $70 \%, 10 \%$ and $20 \%$ respectively. We train the model with the training set, tune hyperparameters with the validation set and report performance on the test set. We crawl user information according to their user IDs via Twitter API.

As we set out to tackle the misinformation detection task, we compare our model with state-of-the-art baselines. They are chosen because they are based on content and/or engagement dynamics, and produce promising performance. RFC [240] is a random forest model with features from the source tweets and engaged user profiles. CRNN [241] combines convolutional recurrent neural networks to extract features from engaged users and retweet texts. CSI [22] incorporates relevant articles and analyses the group behaviour of engaged users. dEFEND [242] uses a co-attention mechanism to study the source claims and user features. The graph-based baseline GCAN has been explained in Related Works. 


\subsection{Results and Analysis}

\subsubsection{Misinformation Classification Performance (RQ1)}

We show the misinformation detection performance of our model against state-ofthe-art baselines on test subsets. From Table 7.3, we can tell that (1) the engagement based models including GCAN and our model beat text content based models on all of the three datasets; (2) our TPP model is able to achieve comparable performance with GCAN. Specifically, we beat GCAN on the Twitter16 dataset and the Pheme dataset in terms of three metrics. This can be explained by the fact that Twitter16 displays greater disparity between the mean spreading of true and false claims, and our model captures such patterns so reach higher performance.

\subsubsection{Time Prediction (RQ2)}

In addition to misinformation detection performance, we are interested in how good our model is at predicting when an engagement occurs during misinformation propagation. Hence we report the mean time prediction error (in minutes) of all engagements on three datasets respectively: 43.16 on Twitter $15,38.75$ on Twitter16 and 52.31 on Pheme. It is noticeable that existing misinformation detection baselines are not able to achieve such functionality.

\subsection{Discussion of Model Deployment}

This proposed model infers claim veracity based on user engagement activities on social platforms. Engagement is a hybrid of user account features, textual replies and temporal and geometric patterns of the affine dynamic graph. When deployed on a social platforms, the main issue comes from the computational complexity. First of all, the model puts demand on disk space to store the affine graph and dynamically update it as more user interacts with a piece of misinformation. Also, the attention-based temporal point processes can be computationally expensive due to the usage of self-attention. The model itself is a sequential one that is hard to parallelize. Therefore, it is required to develop a practically efficient solution to deploy this hybrid method for misinformation detection on social platforms. 


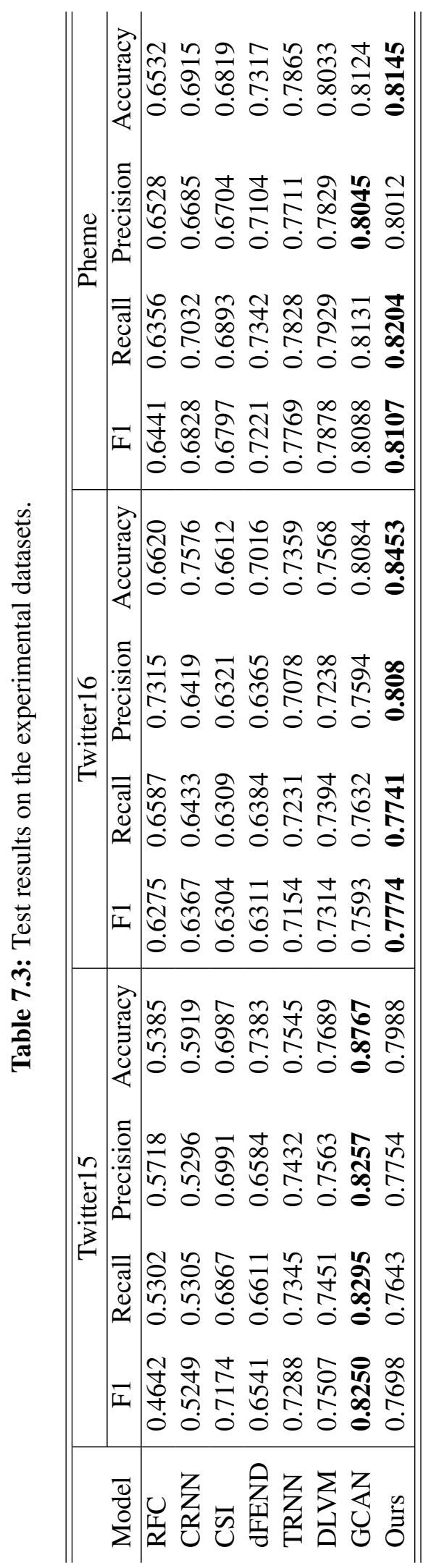




\subsection{Summary}

This study sets out to expand the content-based models to trace the misinformation dynamics. We model the user engagements in misinformation propagation as a continuous-time dynamic graph, and employ Temporal Point Processes to capture geometric and temporal patterns of the graph. A node is actually a timestamped user engagement including all forms of interactions that users conduct with claims on social media platforms. With the temporal evolution of the engagement graph, new engagement nodes will establish edges with existing nodes and thus update the graph. To extract both geometric and temporal engagement features, we view the addition of new engagement nodes as the chronological events and use SAHP to generate the graph embeddings. In this setting, claim veracity evaluation is equivalent to the task of graph classification. With SAHP, we are able to predict when a new engagement will occur next, which has not been studied before. By accumulating the predicted engagements, we are able to quantitatively describe the evolution dynamics of the engagement network. This allows us to forecast the future growth of a claim that has been identified as misinformation, which is valuable to identify the false information that will have enormous mass of readers. This model enables to forecast the growth of the graph scale, which can indicate the level of threat of false information. Experiments show the effectiveness of our model to achieve state-of-the-art performance in misinformation detection and forecasting tasks. 


\section{Chapter 8}

\section{Conclusions \& Future Work}

This thesis aims to explore data-driven machine learning models to evaluate veracity of textual claims on the web. We instantiate claims in two application contexts: online news articles and social media posts. In the former context, we study a news stance detection task and develop a hierarchical stance detection model with a MMD regularization term. While in the latter context, we study how to utilize textual replies from engaged users based on a deep latent variable model and the engagement dynamics based on an attention-modified temporal point process. This chapter summarizes main findings of this thesis and point out potential research directions for the future.

\subsection{Main Findings}

In this thesis, we developed new machine learning algorithms towards automated veracity evaluation and examined their performance in different experimental settings. The main works are listed as follows.

In Chapter 4, we find that there is a semantically hierarchical structure among the four stance classes of news articles, which motivates us to propose a twolayer neural network for the stance detection task. The first layer performs a related/unrelated classification, while the second layer performs a more fine-grained classification among the related classes. The main idea here is to divide a concept into sub-concepts that are organized in a hierarchical structure, and design constraints, i.e., MMD regularization, between sub-concepts in order to make the 
model parameter optimization more sensible. One by-product advantage is that this hierarchical architecture is helpful to overcome the class imbalance problem that often exists in stance datasets. Besides empirical performance improvement, this thesis analytically discusses hierarchical stance detection in the general case of hierarchical machine learning. It is implied that concept abstraction at different levels paves way for the hierarchically structured objects.

Besides news articles, stance is often expressed in social media. Users frequently express their opinions by writing comments or replies towards the claim being verified. In Chapter 5, we instantiate claims as posts on social media platforms, and evaluate claim veracity with engaged user replies. We propose a deep latent variable model, where a latent distribution enables multimodal semantic distribution and more salient credibility feature extraction. This work also confirms the view that incorporating latent probabilistic variables contributes to not only generative but also discriminative deep learning models in terms of allowing multimodal distribution and dealing with semantic ambiguity. After well-calibration, the proposed model is able to provide an uncertainty measurement, which can be used to indicate the confidence level of veracity prediction results.

Textual content based models presented before are not able to trace the dynamics of user engagements in misinformation. In Chapter 6 and Chapter 7, we explore how to identify false information based on engagement dynamics. In Chapter 6, we propose SAHP that incorporates the self-attention mechanism to measure the influence of history events on the occurrence of subsequent events. We also design a time-shifted position embedding method to incorporate the time intervals between events. In Chapter 7, we demonstrate how to modify SAHP for the purpose of user engagement modeling. Previous static engagement graphs ignore temporal information that is inherent to the dissemination process. To overcome this, we build a dynamic engagement graph that is represented as a sequence of time-stamped snapshots, These two works advance the relevant fields by developing a new temporal point process, modeling dynamic engagement graphs and predicting the future threat level of misinformation on the web. 


\subsection{Limitations}

Despite encouraging experimental results, claim veracity evaluation is still a challenging problem and the proposed models in this thesis can have a few limitations. We hereby analyse the limitations of this thesis.

For the news stance detection task in Chapter 4, we analytically and empirically prove the advantage of the hierarchical architecture and the MMD regularization. Due to the scope of this thesis, we did not examine whether the MMD regularization can be well generalized to other hierarchical classification cases. Also, the datasets we use are imbalanced, and it is not clear whether the MMD regularization helps solve the class imbalance problem in general. These issues limit the usage of the MMD regularization in more hierarchical machine learning areas.

Furthermore, the deep latent variable model in Chapter 5 incorporates randomness to veracity evaluation, which is potentially a way of uncertainty measurement. However, the uncertainty has to be calibrated before deployment. It is also not clear how the uncertainty changes as more replies are available, how it is linked to user stances, and how the uncertainty measurement could be used to improve veracity evaluation.

The thesis considers two veracity classes of true and false, while in reality there is an extra class, unverified, as we mention in previous chapters. Excluding the unverified class reduces the complexity of the veracity evaluation task. One consideration of adopting such exclusion is that this thesis sets out to deal with the veracity evaluation task in a more controllable setting. Specifically, we can analyse the pattern difference between claims from true and false classes, without the influence of those unverified ones that can eventually turn to be true or false. It is admitted that excluding the unverified class limits the practical application of machine learning models to real-world social media platforms.

Another limitation comes from the off-line evaluation of the proposed models. All the proposed machine learning models are evaluated in an off-line trainvalidation-test dataset split. The split is fixed and can only indicate the models' performance on a limited number of data snapshots. Without an online evaluation 
setting, we cannot obtain a proper understanding of how models perform, in terms of accuracy and efficiency, in a streaming social media platforms. Besides, we cannot figure out how often we should re-train and update the deployed models to avoid potential biases as more data are available.

\subsection{Future Work}

The research in this thesis has many important implications. In this section, we point out future directions to extend the research of automated veracity evaluation.

\subsubsection{Overcoming Limitations}

The first type of future works is to overcome the limitations of this thesis. As we analyse in Chapter 4, the MMD regularization could been examined in more general cases. It has shown promising performance in improving news stance detection. To extend that, it might be interesting to test MMD in other hierarchical tasks, such as image retrieval and segmentation. Another model modification is to calibrate the uncertainty calculated by the deep latent variable model, based on which we need to do more analytical experiments to understand the uncertainty.

Besides, it is of practical importance to extend the thesis by the incorporating the unverified veracity class. Because unverified claims can eventually turn to be either true or false, there is an overlap in terms of data patterns, such as user replies and engagement dynamics, between unverified and the other two classes. It is therefore expected that data-driven models developed in this thesis could suffer from decreased performance. One promising approach is to firstly distinguish whether a claim is verified or not, then predict truthfulness if a claim is verified. This is quite similar to the hierarchical stance detection model, where we predict relevancy as the first step and then predict the fine-grained agree/disagree/discuss. The hierarchical verification can also be used to examine the MMD regularization.

In terms of experimental design, most existing works, including this thesis, are examined in an off-line fashion. To simulate the practical setting, it is necessary to develop an online simulation system that is analogous to real-world social media platforms. The preference of developing a simulation system over straight- 
forwardly employing real-world platforms is due to a simpler and more controllable setting: researchers can adjust the experiment environment and parameters according to their needs. The results can be more interpretable. After that, it is necessary to deploy models in real-world platforms and test their accuracy and efficiency.

\subsubsection{Expanding Research}

It is important to develop more effective models to utilize features from various aspects for veracity evaluation. As discussed in Literature Review, a number of aspects can indicate veracity, such as textual and visual content, social comments, engagement dynamics, and source authenticity. Existing models, including the hybrid model in Chapter 7, incorporate features from these aspects into one single model. This might not be an effective way as each feature has its own limitations in terms of veracity prediction. To overcome this deficit, one promising direction is to develop ensemble models. This is based on the fact that ensemble models construct a set of weak machine learners for a stronger learner that is more capable than any individual weak one. Hence it might be advantageous to have weak learners for supplementary aspects to boost the ensemble performance.

Annotated datasets of false information can be domain-dependent. This is because that misinformation exists in almost all kinds of domains, be it political, financial and health-related. News from different domains have different writing styles, different sets of vocabulary. Also, false information may have event or topic specific keywords and phrases. Due to the problem of diversified content, automatic veracity classifiers trained with one domain dataset have difficulties in generalizing to another domain where event, topic or vocabulary is different. Furthermore, it is infeasible to train separate classifiers for each domain, because the annotation process is both time and resource consuming, and may also require domain-knowledge experts. How to learn salient features across domains is an interesting direction.

Last but not least, research needs to be done on data-efficient machine learning. The majority of current veracity evaluation models are supervised machine learning, most commonly deep learning, that requires a large-scale annotated datasets to enable model training. Notwithstanding, accurately annotating ground truth of news 
claims is quite time-consuming and labor-intensive. This is because the annotation process requires domain experts carefully analysing the claim being verified and relevant evidences, reports, context or legislate documents from authentic sources. Moreover, the dataset might need to be regularly updated to ensure well-curated ground truth. Therefore, it is vital to look into data-efficient machine learning cases where a limited number of annotations are demanded. Semi-supervised, transfer and meta learning are more feasible in industry practice.

Apart from technical considerations, it is important to analyse ethical concerns of data-driven misinformation detection models. One is about private data leakage and misuse. Existing models extract various types of features such as user profiles and activity histories. Such a feature extraction process requires wide, if not full, access to user data on the web. The model developer, which can be the social big-tech, the government agencies or third-parties, may take unauthorized actions in utilizing these data. Another concern is algorithmic biases due to personalized content. As we mentioned, we can alleviate data bias by collecting more data and frequently updating the trained model. However, due to personalized recommendations, people are often exposed to what they are in favour of, and have little access to views they disagree. This algorithmic operations deepen peoples' bias and more diverse content should be recommended. Finally, it is crucial to consider how to enjoy the nuances of digital media without hurting fairness and democracy. For example, it might be against democratic values to suspend a user account just because he or she frequently shares extremely left or right views that are often verified to be false. Hence, data-driven misinformation detection models need pay attention on how to promote fairness and democracy within society. 


\section{Appendix A}

\section{Full List of Publications}

Qiang Zhang has published the following conference papers during his Ph.D study.

- Q Zhang, J Cook and E Yilmaz. Detecting and Forecasting Misinformation via Temporal and Geometric Propagation Patterns. ECIR, Norway, 2021. (Chapter 6, short paper)

- Q Zhang, A Lipani and E Yilmaz. Learning Neural Point Processes with Latent Graphs. WWW, Slovenia, 2021.

- Q Zhang, A Lipani, O Kirnap and E Yilmaz. Self-Attentive Hawkes Process. ICML, Vienna Austria, 2020. (Chapter 5)

- Q Zhang, R Luo, Y Yang and J Wang. Replica-Exchange Nosé-Hoover Dynamics for Bayesian Learning on Large Datasets. NeurIPS, 2020.

- Q Zhang, A Lipani, S Liang and E Yilmaz. Reply-Aided Detection of Misinformation via Bayesian Deep Learning. WWW, San Francisco, 2019. (Chapter 4)

- Q Zhang, S Liang, A Lipani and E Yilmaz. From Stances' Imbalance to Their Hierarchical Representation and Detection. WWW, San Francisco, 2019. (Chapter 3)

- Q Zhang, S Liang and E Yilmaz. Variational Self-Attention Model for Sentence Representation, NeurIPS workshop on Bayesian Deep Learning, Montréal Canada, 2018. 
- Q Zhang, R Luo, Y Yang, Y Liu. Parallel-tempered Stochastic Gradient Hamiltonian Monte Carlo for Approximate Multimodal Posterior Sampling. NeurIPS Symposium on Advances in Approximate Bayesian Inference, Montréal Canada, 2018.

- Q Zhang, E Yilmaz and S Liang. Ranking-based method for news stance detection. WWW, Lyon France, 2018. (Short Paper) 


\section{Bibliography}

[1] Srijan Kumar, Robert West, and Jure Leskovec. Disinformation on the web: Impact, characteristics, and detection of wikipedia hoaxes. In Proceedings of the 25th international conference on World Wide Web, pages 591-602. International World Wide Web Conferences Steering Committee, 2016.

[2] Kiran Garimella, Gianmarco De Francisci Morales, Aristides Gionis, and Michael Mathioudakis. Reducing controversy by connecting opposing views. In Proceedings of the Tenth ACM International Conference on Web Search and Data Mining, WSDM 2017, Cambridge, United Kingdom, February 610, 2017, pages 81-90, 2017.

[3] Preslav Nakov, Giovanni Da San Martino, Tamer Elsayed, Alberto BarrónCedeño, Rubén Míguez, Shaden Shaar, Firoj Alam, Fatima Haouari, Maram Hasanain, Nikolay Babulkov, Alex Nikolov, Gautam Kishore Shahi, Julia Maria Struß, and Thomas Mandl. The clef-2021 checkthat! lab on detecting check-worthy claims, previously fact-checked claims, and fake news. In Djoerd Hiemstra, Marie-Francine Moens, Josiane Mothe, Raffaele Perego, Martin Potthast, and Fabrizio Sebastiani, editors, Advances in Information Retrieval, pages 639-649, Cham, 2021. Springer International Publishing.

[4] Yann LeCun, Léon Bottou, Yoshua Bengio, and Patrick Haffner. Gradientbased learning applied to document recognition. Proceedings of the IEEE, 86(11):2278-2324, 1998.

[5] Yann LeCun, Yoshua Bengio, and Geoffrey Hinton. Deep learning. nature, 521(7553):436, 2015. 
[6] Ashish Vaswani, Noam Shazeer, Niki Parmar, Jakob Uszkoreit, Llion Jones, Aidan N. Gomez, Lukasz Kaiser, and Illia Polosukhin. Attention is all you need. In Advances in Neural Information Processing Systems 30: Annual Conference on Neural Information Processing Systems 2017, 4-9 December 2017, Long Beach, CA, USA, pages 6000-6010, 2017.

[7] Rohan Chandra, Sachin Grover, Kyungjun Lee, Moustafa Meshry, and Ahmed Taha. Texture synthesis with recurrent variational auto-encoder. arXiv preprint arXiv:1712.08838, 2017.

[8] Anatoliy Swishchuk. Hawkes processes and their applications in finance and insurance. Talk from the Department of Mathematics and Statistics of the University of Calgary. Available online at: people. ucalgary. ca/aswish/Swishchuk_LaLTalk. pdf(accessed June 10 2019.), 2017.

[9] Andrew Perrin. Social media usage. Pew research center, pages 52-68, 2015.

[10] Jeffrey Gottfried and Elisa Shearer. News Use Across Social Medial Platforms 2016. Pew Research Center, 2016.

[11] Soroush Vosoughi, Deb Roy, and Sinan Aral. The spread of true and false news online. Science, 359(6380):1146-1151, 2018.

[12] Adrien Friggeri, Lada A. Adamic, Dean Eckles, and Justin Cheng. Rumor cascades. In Proceedings of the Eighth International Conference on Weblogs and Social Media, ICWSM 2014, Ann Arbor, Michigan, USA, June 1-4, 2014., 2014.

[13] Todor Mihaylov, Georgi Georgiev, and Preslav Nakov. Finding opinion manipulation trolls in news community forums. In Proceedings of the nineteenth conference on computational natural language learning, pages 310$314,2015$.

[14] Todor Mihaylov and Preslav Nakov. Hunting for troll comments in news community forums. In Proceedings of the 54th Annual Meeting of the Asso- 
ciation for Computational Linguistics (Volume 2: Short Papers), volume 2, pages 399-405, 2016.

[15] Johan Bollen, Huina Mao, and Xiao-Jun Zeng. Twitter mood predicts the stock market. J. Comput. Science, 2(1):1-8, 2011.

[16] Aditi Gupta, Hemank Lamba, Ponnurangam Kumaraguru, and Anupam Joshi. Faking sandy: characterizing and identifying fake images on twitter during hurricane sandy. In Proceedings of the 22nd international conference on World Wide Web, pages 729-736. ACM, 2013.

[17] Kai Shu, Amy Sliva, Suhang Wang, Jiliang Tang, and Huan Liu. Fake news detection on social media: A data mining perspective. SIGKDD Explorations, 19(1):22-36, 2017.

[18] Natali Ruchansky, Sungyong Seo, and Yan Liu. CSI: A hybrid deep model for fake news detection. In Proceedings of the 2017 ACM on Conference on Information and Knowledge Management, CIKM 2017, Singapore, November 06 - 10, 2017, pages 797-806, 2017.

[19] Naeemul Hassan, Chengkai Li, and Mark Tremayne. Detecting checkworthy factual claims in presidential debates. In Proceedings of the 24th ACM International on Conference on Information and Knowledge Management, pages 1835-1838. ACM, 2015.

[20] Qiang Zhang, Emine Yilmaz, and Shangsong Liang. Ranking-based method for news stance detection. In Companion Proceedings of the The Web Conference 2018. ACM Press, 2018.

[21] Jiliang Tang, Yi Chang, and Huan Liu. Mining social media with social theories: a survey. ACM Sigkdd Explorations Newsletter, 15(2):20-29, 2014.

[22] Natali Ruchansky, Sungyong Seo, and Yan Liu. Csi: A hybrid deep model for fake news detection. In Proceedings of the 2017 ACM on Conference on Information and Knowledge Management, pages 797-806. ACM, 2017. 
[23] Qi Huang, Junshuai Yu, Jia Wu, and Bin Wang. Heterogeneous graph attention networks for early detection of rumors on twitter. arXiv preprint arXiv:2006.05866, 2020.

[24] Jing Ma, Wei Gao, and Kam-Fai Wong. Rumor detection on twitter with tree-structured recursive neural networks. In Proceedings of the 56th Annual Meeting of the Association for Computational Linguistics (Volume 1: Long Papers), volume 1, pages 1980-1989, 2018.

[25] Elena Kochkina, Maria Liakata, and Arkaitz Zubiaga. All-in-one: Multi-task learning for rumour verification. In Proceedings of the 27th International Conference on Computational Linguistics, COLING 2018, Santa Fe, New Mexico, USA, August 20-26, 2018, pages 3402-3413, 2018.

[26] Kai Shu, Amy Sliva, Suhang Wang, Jiliang Tang, and Huan Liu. Fake news detection on social media: A data mining perspective. ACM SIGKDD Explorations Newsletter, 19(1):22-36, 2017.

[27] Jacquelyn Rahman. The $\mathrm{n}$ word: Its history and use in the african american community. Journal of English Linguistics, 40(2):137-171, 2012.

[28] James Thorne and Andreas Vlachos. Automated fact checking: Task formulations, methods and future directions. In Proceedings of the 27th International Conference on Computational Linguistics, pages 3346-3359. Association for Computational Linguistics, 2018.

[29] Myle Ott, Yejin Choi, Claire Cardie, and Jeffrey T. Hancock. Finding deceptive opinion spam by any stretch of the imagination. In The 49th Annual Meeting of the Association for Computational Linguistics: Human Language Technologies, Proceedings of the Conference, 19-24 June, 2011, Portland, Oregon, USA, pages 309-319, 2011.

[30] Verónica Pérez-Rosas, Bennett Kleinberg, Alexandra Lefevre, and Rada Mihalcea. Automatic detection of fake news. In Proceedings of the 27th In- 
ternational Conference on Computational Linguistics, COLING 2018, Santa Fe, New Mexico, USA, August 20-26, 2018, pages 3391-3401, 2018.

[31] Yuanshun Yao, Bimal Viswanath, Jenna Cryan, Haitao Zheng, and Ben Y. Zhao. Automated crowdturfing attacks and defenses in online review systems. In Proceedings of the 2017 ACM SIGSAC Conference on Computer and Communications Security, CCS 2017, Dallas, TX, USA, October 30 November 03, 2017, pages 1143-1158, 2017.

[32] Edward S Reed, Elliot Turiel, and Terrance Brown. Naive realism in everyday life: Implications for social conflict and misunderstanding. In Values and knowledge, pages 113-146. Psychology Press, 2013.

[33] Raymond S Nickerson. Confirmation bias: A ubiquitous phenomenon in many guises. Review of general psychology, 2(2):175, 1998.

[34] Solomon E Asch and H Guetzkow. Effects of group pressure upon the modification and distortion of judgments. Groups, leadership, and men, pages 222-236, 1951.

[35] Gordon Pennycook and David G Rand. Who falls for fake news? the roles of bullshit receptivity, overclaiming, familiarity, and analytic thinking. 2018.

[36] Scott F Aikin. Poe's law, group polarization, and the epistemology of online religious discourse. 2009.

[37] Hunt Allcott and Matthew Gentzkow. Social media and fake news in the 2016 election. Journal of Economic Perspectives, 31(2):211-36, 2017.

[38] Michela Del Vicario, Alessandro Bessi, Fabiana Zollo, Fabio Petroni, Antonio Scala, Guido Caldarelli, H Eugene Stanley, and Walter Quattrociocchi. The spreading of misinformation online. Proceedings of the National Academy of Sciences, 113(3):554-559, 2016.

[39] Walter Quattrociocchi, Antonio Scala, and Cass R Sunstein. Echo chambers on facebook. 2016. 
[40] Andreas Vlachos and Sebastian Riedel. Fact checking: Task definition and dataset construction. In Proceedings of the ACL 2014 Workshop on Language Technologies and Computational Social Science, pages 18-22, 2014.

[41] Yimin Chen, Niall J Conroy, and Victoria L Rubin. Misleading online content: Recognizing clickbait as false news. In Proceedings of the 2015 ACM on Workshop on Multimodal Deception Detection, pages 15-19. ACM, 2015.

[42] Song Feng, Ritwik Banerjee, and Yejin Choi. Syntactic stylometry for deception detection. In Proceedings of the 50th Annual Meeting of the Association for Computational Linguistics: Short Papers-Volume 2, pages 171-175. Association for Computational Linguistics, 2012.

[43] Victoria L Rubin and Tatiana Lukoianova. Truth and deception at the rhetorical structure level. Journal of the Association for Information Science and Technology, 66(5):905-917, 2015.

[44] Johannes Fürnkranz. A study using n-gram features for text categorization. Austrian Research Institute for Artifical Intelligence, 3(1998):1-10, 1998.

[45] Martin Potthast, Johannes Kiesel, Kevin Reinartz, Janek Bevendorff, and Benno Stein. A stylometric inquiry into hyperpartisan and fake news. arXiv preprint arXiv:1702.05638, 2017.

[46] Nayeon Lee, Yejin Bang, Andrea Madotto, and Pascale Fung. Misinformation has high perplexity. arXiv preprint arXiv:2006.04666, 2020.

[47] Yimin Chen, Niall J. Conroy, and Victoria L. Rubin. Misleading online content: Recognizing clickbait as "false news". In Proceedings of the 2015 ACM Workshop on Multimodal Deception Detection,WMDD@ICMI 2015, Seattle, Washington, USA, November 13, 2015, pages 15-19, 2015.

[48] Sejeong Kwon, Meeyoung Cha, Kyomin Jung, Wei Chen, et al. Prominent features of rumor propagation in online social media. In International Conference on Data Mining. IEEE, 2013. 
[49] Hannah Rashkin, Eunsol Choi, Jin Yea Jang, Svitlana Volkova, and Yejin Choi. Truth of varying shades: Analyzing language in fake news and political fact-checking. In Proceedings of the 2017 conference on empirical methods in natural language processing, pages 2931-2937, 2017.

[50] Suhang Wang, Jiliang Tang, Charu Aggarwal, and Huan Liu. Linked document embedding for classification. In Proceedings of the 25th ACM international on conference on information and knowledge management, pages 115-124. ACM, 2016.

[51] Suhang Wang, Jiliang Tang, Fred Morstatter, and Huan Liu. Paired restricted boltzmann machine for linked data. In Proceedings of the 25th ACM International on Conference on Information and Knowledge Management, pages 1753-1762. ACM, 2016.

[52] Jacob Devlin, Ming-Wei Chang, Kenton Lee, and Kristina Toutanova. Bert: Pre-training of deep bidirectional transformers for language understanding. arXiv preprint arXiv:1810.04805, 2018.

[53] Tom B Brown, Benjamin Mann, Nick Ryder, Melanie Subbiah, Jared Kaplan, Prafulla Dhariwal, Arvind Neelakantan, Pranav Shyam, Girish Sastry, Amanda Askell, et al. Language models are few-shot learners. arXiv preprint arXiv:2005.14165, 2020.

[54] Ndapandula Nakashole and Tom M Mitchell. Language-aware truth assessment of fact candidates. In Proceedings of the 52nd Annual Meeting of the Association for Computational Linguistics (Volume 1: Long Papers), volume 1, pages 1009-1019, 2014.

[55] Luciano Floridi. Artificial intelligence, deepfakes and a future of ectypes. Philosophy \& Technology, 31(3):317-321, 2018.

[56] Zhiwei Jin, Juan Cao, Yongdong Zhang, Jianshe Zhou, and Qi Tian. Novel visual and statistical image features for microblogs news verification. IEEE transactions on multimedia, 19(3):598-608, 2017. 
[57] Yang Yang, Lei Zheng, Jiawei Zhang, Qingcai Cui, Zhoujun Li, and Philip S Yu. Ti-cnn: Convolutional neural networks for fake news detection. arXiv preprint arXiv:1806.00749, 2018.

[58] Minyoung Huh, Andrew Liu, Andrew Owens, and Alexei A Efros. Fighting fake news: Image splice detection via learned self-consistency. In Proceedings of the European Conference on Computer Vision (ECCV), pages 101-117, 2018.

[59] Dhruv Khattar, Jaipal Singh Goud, Manish Gupta, and Vasudeva Varma. Mvae: Multimodal variational autoencoder for fake news detection. In The World Wide Web Conference, pages 2915-2921, 2019.

[60] Shengsheng Qian, Jinguang Wang, Jun Hu, Quan Fang, and Changsheng Xu. Hierarchical multi-modal contextual attention network for fake news detection. In Proceedings of the 44th International ACM SIGIR Conference on Research and Development in Information Retrieval, pages 153-162, 2021.

[61] You Wu, Pankaj K Agarwal, Chengkai Li, Jun Yang, and Cong Yu. Toward computational fact-checking. Proceedings of the VLDB Endowment, 7(7):589-600, 2014.

[62] Giovanni Luca Ciampaglia, Prashant Shiralkar, Luis M Rocha, Johan Bollen, Filippo Menczer, and Alessandro Flammini. Computational fact checking from knowledge networks. PloS one, 10(6):e0128193, 2015.

[63] Baoxu Shi and Tim Weninger. Fact checking in heterogeneous information networks. In Proceedings of the 25th International Conference Companion on World Wide Web, pages 101-102. International World Wide Web Conferences Steering Committee, 2016.

[64] James Thorne and Andreas Vlachos. An extensible framework for verification of numerical claims. In Proceedings of the Software Demonstrations of the 15th Conference of the European Chapter of the Association for Computational Linguistics, pages 37-40, 2017. 
[65] James Thorne, Andreas Vlachos, Christos Christodoulopoulos, and Arpit Mittal. FEVER: a large-scale dataset for fact extraction and verification. In NAACL-HLT, 2018.

[66] Michele Banko, Michael J Cafarella, Stephen Soderland, Matthew Broadhead, and Oren Etzioni. Open information extraction from the web. In IJCAI, volume 7, pages 2670-2676, 2007.

[67] Amr Magdy and Nayer Wanas. Web-based statistical fact checking of textual documents. In Proceedings of the 2nd international workshop on Search and mining user-generated contents, pages 103-110. ACM, 2010.

[68] Naeemul Hassan, Gensheng Zhang, Fatma Arslan, Josue Caraballo, Damian Jimenez, Siddhant Gawsane, Shohedul Hasan, Minumol Joseph, Aaditya Kulkarni, Anil Kumar Nayak, et al. Claimbuster: The first-ever end-to-end fact-checking system. Proceedings of the VLDB Endowment, 10(12):1945$1948,2017$.

[69] William Ferreira and Andreas Vlachos. Emergent: a novel data-set for stance classification. In NAACL HLT 2016, The 2016 Conference of the North American Chapter of the Association for Computational Linguistics: Human Language Technologies, San Diego California, USA, June 12-17, 2016, pages 1163-1168, 2016.

[70] Hoa Trang Dang, Diane Kelly, and Jimmy J Lin. Overview of the trec 2007 question answering track. In Trec, volume 7, page 63, 2007.

[71] Samuel R Bowman, Gabor Angeli, Christopher Potts, and Christopher D Manning. A large annotated corpus for learning natural language inference. arXiv preprint arXiv:1508.05326, 2015.

[72] Ankur P Parikh, Oscar Täckström, Dipanjan Das, and Jakob Uszkoreit. A decomposable attention model for natural language inference. arXiv preprint arXiv:1606.01933, 2016. 
[73] Qian Chen, Xiaodan Zhu, Zhenhua Ling, Si Wei, Hui Jiang, and Diana Inkpen. Enhanced lstm for natural language inference. arXiv preprint arXiv:1609.06038, 2016.

[74] Max Glockner, Vered Shwartz, and Yoav Goldberg. Breaking nli systems with sentences that require simple lexical inferences. arXiv preprint arXiv:1805.02266, 2018.

[75] Xinyu Hua and Lu Wang. Understanding and detecting supporting arguments of diverse types. arXiv preprint arXiv:1705.00045, 2017.

[76] Carlos Castillo, Marcelo Mendoza, and Barbara Poblete. Information credibility on twitter. In Proceedings of the 20th international conference on World wide web, pages 675-684. ACM, 2011.

[77] Fan Yang, Yang Liu, Xiaohui Yu, and Min Yang. Automatic detection of rumor on sina weibo. In Proceedings of the ACM SIGKDD Workshop on Mining Data Semantics, page 13. ACM, 2012.

[78] Jing Ma, Wei Gao, Zhongyu Wei, Yueming Lu, and Kam-Fai Wong. Detect rumors using time series of social context information on microblogging websites. In Proceedings of the 24th ACM International on Conference on Information and Knowledge Management, pages 1751-1754. ACM, 2015.

[79] Jing Ma, Wei Gao, Prasenjit Mitra, Sejeong Kwon, Bernard J Jansen, KamFai Wong, and Meeyoung Cha. Detecting rumors from microblogs with recurrent neural networks. In IJCAI, pages 3818-3824, 2016.

[80] Michal Lukasik, PK Srijith, Duy Vu, Kalina Bontcheva, Arkaitz Zubiaga, and Trevor Cohn. Hawkes processes for continuous time sequence classification: an application to rumour stance classification in twitter. In Proceedings of the 54th Annual Meeting of the Association for Computational Linguistics (Volume 2: Short Papers), volume 2, pages 393-398, 2016. 
[81] Zhiwei Jin, Juan Cao, Yongdong Zhang, and Jiebo Luo. News verification by exploiting conflicting social viewpoints in microblogs. In $A A A I$, pages 2972-2978, 2016.

[82] Eugenio Tacchini, Gabriele Ballarin, Marco L Della Vedova, Stefano Moret, and Luca de Alfaro. Some like it hoax: Automated fake news detection in social networks. arXiv preprint arXiv:1704.07506, 2017.

[83] Zhiwei Jin, Juan Cao, Yu-Gang Jiang, and Yongdong Zhang. News credibility evaluation on microblog with a hierarchical propagation model. In 2014 IEEE International Conference on Data Mining, pages 230-239. IEEE, 2014.

[84] Manish Gupta, Peixiang Zhao, and Jiawei Han. Evaluating event credibility on twitter. In Proceedings of the 2012 SIAM International Conference on Data Mining, pages 153-164. SIAM, 2012.

[85] Chaoyuan Zuo, Ayla Karakas, and Ritwik Banerjee. A hybrid recognition system for check-worthy claims using heuristics and supervised learning. In CLEF (Working Notes), 2018.

[86] Fatma Arslan, Naeemul Hassan, Chengkai Li, and Mark Tremayne. A benchmark dataset of check-worthy factual claims. In Proceedings of the International AAAI Conference on Web and Social Media, volume 14, pages 821$829,2020$.

[87] Gullal S Cheema, Sherzod Hakimov, and Ralph Ewerth. Check_square at checkthat! 2020: Claim detection in social media via fusion of transformer and syntactic features. arXiv preprint arXiv:2007.10534, 2020.

[88] Tomas Mikolov, Edouard Grave, Piotr Bojanowski, Christian Puhrsch, and Armand Joulin. Advances in pre-training distributed word representations. arXiv preprint arXiv:1712.09405, 2017. 
[89] Saif M Mohammad, Parinaz Sobhani, and Svetlana Kiritchenko. Stance and sentiment in tweets. ACM Transactions on Internet Technology (TOIT), 17(3):26, 2017.

[90] Arkaitz Zubiaga, Maria Liakata, Rob Procter, Geraldine Wong Sak Hoi, and Peter Tolmie. Analysing how people orient to and spread rumours in social media by looking at conversational threads. PloS one, 11(3):e0150989, 2016.

[91] Benjamin Riedel, Isabelle Augenstein, Georgios P Spithourakis, and Sebastian Riedel. A simple but tough-to-beat baseline for the fake news challenge stance detection task. arXiv preprint arXiv:1707.03264, 2017.

[92] Andreas Hanselowski, Avinesh PVS, Benjamin Schiller, Felix Caspelherr, Debanjan Chaudhuri, Christian M Meyer, and Iryna Gurevych. A retrospective analysis of the fake news challenge stance detection task. arXiv preprint arXiv:1806.05180, 2018.

[93] James Thorne, Andreas Vlachos, Christos Christodoulopoulos, and Arpit Mittal. Fever: a large-scale dataset for fact extraction and verification. arXiv preprint arXiv:1803.05355, 2018.

[94] L. Derczynski, K. Bontcheva, M. Liakata, R. Procter, G.W.S. Hoi, and A. Zubiaga. Semeval-2017 task 8: Rumoureval: Determining rumour veracity and support for rumours, August 2017. C 2017 Association for Computational Linguistics.

[95] Momchil Hardalov, Arnav Arora, Preslav Nakov, and Isabelle Augenstein. A survey on stance detection for mis-and disinformation identification. arXiv preprint arXiv:2103.00242, 2021.

[96] Saif Mohammad, Svetlana Kiritchenko, Parinaz Sobhani, Xiaodan Zhu, and Colin Cherry. Semeval-2016 task 6: Detecting stance in tweets. In Proceedings of the 10th International Workshop on Semantic Evaluation (SemEval2016), pages 31-41, 2016. 
[97] Elena Kochkina, Maria Liakata, and Arkaitz Zubiaga. All-in-one: Multi-task learning for rumour verification. In Proceedings of the 27th International Conference on Computational Linguistics, pages 3402-3413. Association for Computational Linguistics, 2018.

[98] Arkaitz Zubiaga, Ahmet Aker, Kalina Bontcheva, Maria Liakata, and Rob Procter. Detection and resolution of rumours in social media: A survey. ACM Computing Surveys (CSUR), 51(2):32, 2018.

[99] Liang Wu, Jundong Li, Xia Hu, and Huan Liu. Gleaning wisdom from the past: Early detection of emerging rumors in social media. In Proceedings of the 2017 SIAM International Conference on Data Mining, pages 99-107. SIAM, 2017.

[100] Justin Sampson, Fred Morstatter, Liang Wu, and Huan Liu. Leveraging the implicit structure within social media for emergent rumor detection. In Proceedings of the 25th ACM International on Conference on Information and Knowledge Management, pages 2377-2382. ACM, 2016.

[101] Yaliang Li, Jing Gao, Chuishi Meng, Qi Li, Lu Su, Bo Zhao, Wei Fan, and Jiawei Han. A survey on truth discovery. ACM Sigkdd Explorations Newsletter, 17(2):1-16, 2016.

[102] Subhabrata Mukherjee and Gerhard Weikum. Leveraging joint interactions for credibility analysis in news communities. In Proceedings of the 24th ACM International on Conference on Information and Knowledge Management, pages 353-362. ACM, 2015.

[103] Gerhard Weikum. What computers should know, shouldn't know, and shouldn't believe. In Proceedings of the 26th International Conference on World Wide Web Companion, pages 1559-1560. International World Wide Web Conferences Steering Committee, 2017.

[104] Carl I Hovland and Walter Weiss. The influence of source credibility on communication effectiveness. Public opinion quarterly, 15(4):635-650, 1951. 
[105] Chanthika Pornpitakpan. The persuasiveness of source credibility: A critical review of five decades' evidence. Journal of applied social psychology, 34(2):243-281, 2004.

[106] Shawn Tseng and BJ Fogg. Credibility and computing technology. Communications of the ACM, 42(5):39-44, 1999.

[107] Robert Thomson, Naoya Ito, Hinako Suda, Fangyu Lin, Yafei Liu, Ryo Hayasaka, Ryuzo Isochi, and Zian Wang. Trusting tweets: The fukushima disaster and information source credibility on twitter. In Proceedings of the 9th International ISCRAM Conference, pages 1-10. Vancouver: Simon Fraser University, 2012.

[108] Emilio Ferrara, Onur Varol, Clayton Davis, Filippo Menczer, and Alessandro Flammini. The rise of social bots. Communications of the ACM, 59(7):96104, 2016.

[109] Fred Morstatter, Harsh Dani, Justin Sampson, and Huan Liu. Can one tamper with the sample api?: Toward neutralizing bias from spam and bot content. In Proceedings of the 25th International Conference Companion on World Wide Web, pages 81-82. International World Wide Web Conferences Steering Committee, 2016.

[110] Fred Morstatter, Liang Wu, Tahora H Nazer, Kathleen M Carley, and Huan Liu. A new approach to bot detection: striking the balance between precision and recall. In 2016 IEEE/ACM International Conference on Advances in Social Networks Analysis and Mining (ASONAM), pages 533-540. IEEE, 2016.

[111] William Ferreira and Andreas Vlachos. Emergent: a novel data-set for stance classification. In Proceedings of the 2016 Conference of the North American Chapter of the Association for Computational Linguistics: Human Language Technologies. ACL, 2016. 
[112] Craig Silverman. Lies, damn lies, and viral content: How news websites spread (and debunk) online rumors, unverified claims and misinformation. Tow Center for Digital Journalism, 168, 2015.

[113] Jing Ma, Wei Gao, and Kam-Fai Wong. Detect rumor and stance jointly by neural multi-task learning. In Companion of the The Web Conference 2018 on The Web Conference 2018, pages 585-593. International World Wide Web Conferences Steering Committee, 2018.

[114] Alessandro Bessi and Emilio Ferrara. Social bots distort the 2016 us presidential election online discussion. 2016.

[115] Philip N Howard and Bence Kollanyi. Bots,\# strongerin, and\# brexit: computational propaganda during the uk-eu referendum. 2016.

[116] Fang Jin, Edward R. Dougherty, Parang Saraf, Yang Cao, and Naren Ramakrishnan. Epidemiological modeling of news and rumors on twitter. In Proceedings of the 7th Workshop on Social Network Mining and Analysis, SNAKDD 2013, Chicago, IL, USA, August 11, 2013, pages 8:1-8:9, 2013.

[117] Jooyeon Kim, Behzad Tabibian, Alice Oh, Bernhard Schölkopf, and Manuel Gomez-Rodriguez. Leveraging the crowd to detect and reduce the spread of fake news and misinformation. In Proceedings of the Eleventh ACM International Conference on Web Search and Data Mining, WSDM 2018, Marina Del Rey, CA, USA, February 5-9, 2018, pages 324-332, 2018.

[118] Marcelo Mendoza, Barbara Poblete, and Carlos Castillo. Twitter under crisis: Can we trust what we rt? In Proceedings of the first workshop on social media analytics, pages 71-79. ACM, 2010.

[119] Tanushree Mitra and Eric Gilbert. Credbank: A large-scale social media corpus with associated credibility annotations. In ICWSM, pages 258-267, 2015. 
[120] Tanushree Mitra, Graham P Wright, and Eric Gilbert. A parsimonious language model of social media credibility across disparate events. In Proceedings of the 2017 ACM Conference on Computer Supported Cooperative Work and Social Computing, pages 126-145. ACM, 2017.

[121] A Conrad Nied, Leo Stewart, Emma Spiro, and Kate Starbird. Alternative narratives of crisis events: Communities and social botnets engaged on social media. In Companion of the 2017 ACM Conference on Computer Supported Cooperative Work and Social Computing, pages 263-266. ACM, 2017.

[122] Vahed Qazvinian, Emily Rosengren, Dragomir R Radev, and Qiaozhu Mei. Rumor has it: Identifying misinformation in microblogs. In Proceedings of the Conference on Empirical Methods in Natural Language Processing, pages 1589-1599. Association for Computational Linguistics, 2011.

[123] Neil Shah, Hemank Lamba, Alex Beutel, and Christos Faloutsos. The many faces of link fraud. In Data Mining (ICDM), 2017 IEEE International Conference on, pages 1069-1074. IEEE, 2017.

[124] Chengcheng Shao, Giovanni Luca Ciampaglia, Alessandro Flammini, and Filippo Menczer. Hoaxy: A platform for tracking online misinformation. In Proceedings of the 25th international conference companion on world wide web, pages 745-750. International World Wide Web Conferences Steering Committee, 2016.

[125] Chengcheng Shao, Giovanni Luca Ciampaglia, Onur Varol, Alessandro Flammini, and Filippo Menczer. The spread of fake news by social bots. arXiv preprint arXiv:1707.07592, 2017.

[126] Kate Starbird. Examining the alternative media ecosystem through the production of alternative narratives of mass shooting events on twitter. In ICWSM, pages 230-239, 2017.

[127] Kate Starbird, Jim Maddock, Mania Orand, Peg Achterman, and Robert M Mason. Rumors, false flags, and digital vigilantes: Misinformation on twitter 
after the 2013 boston marathon bombing. IConference 2014 Proceedings, 2014.

[128] Rudra M Tripathy, Amitabha Bagchi, and Sameep Mehta. A study of rumor control strategies on social networks. In Proceedings of the 19th ACM international conference on Information and knowledge management, pages 1817-1820. ACM, 2010.

[129] Li Zeng, Kate Starbird, and Emma S Spiro. Rumors at the speed of light? modeling the rate of rumor transmission during crisis. In System Sciences (HICSS), 2016 49th Hawaii International Conference on, pages 1969-1978. IEEE, 2016.

[130] VS Subrahmanian, Amos Azaria, Skylar Durst, Vadim Kagan, Aram Galstyan, Kristina Lerman, Linhong Zhu, Emilio Ferrara, Alessandro Flammini, Filippo Menczer, et al. The darpa twitter bot challenge. arXiv preprint arXiv:1601.05140, 2016.

[131] Arkaitz Zubiaga, Geraldine Wong Sak Hoi, Maria Liakata, Rob Procter, and Peter Tolmie. Analysing how people orient to and spread rumours in social media by looking at conversational threads. CoRR, abs/1511.07487, 2015.

[132] Alex Beutel, Wanhong Xu, Venkatesan Guruswami, Christopher Palow, and Christos Faloutsos. Copycatch: stopping group attacks by spotting lockstep behavior in social networks. In Proceedings of the 22nd international conference on World Wide Web, pages 119-130. ACM, 2013.

[133] Nam P Nguyen, Guanhua Yan, My T Thai, and Stephan Eidenbenz. Containment of misinformation spread in online social networks. In Proceedings of the 4th Annual ACM Web Science Conference, pages 213-222. ACM, 2012.

[134] Meng Jiang, Peng Cui, Alex Beutel, Christos Faloutsos, and Shiqiang Yang. Inferring strange behavior from connectivity pattern in social networks. In Pacific-Asia Conference on Knowledge Discovery and Data Mining, pages 126-138. Springer, 2014. 
[135] Jooyeon Kim, Behzad Tabibian, Alice Oh, Bernhard Schölkopf, and Manuel Gomez-Rodriguez. Leveraging the crowd to detect and reduce the spread of fake news and misinformation. In Proceedings of the Eleventh ACM International Conference on Web Search and Data Mining, pages 324-332. ACM, 2018.

[136] Ke Wu, Song Yang, and Kenny Q Zhu. False rumors detection on sina weibo by propagation structures. In Data Engineering (ICDE), 2015 IEEE 31st International Conference on, pages 651-662. IEEE, 2015.

[137] Savvas Zannettou, Tristan Caulfield, Emiliano De Cristofaro, Nicolas Kourtelris, Ilias Leontiadis, Michael Sirivianos, Gianluca Stringhini, and Jeremy Blackburn. The web centipede: understanding how web communities influence each other through the lens of mainstream and alternative news sources. In Proceedings of the 2017 Internet Measurement Conference, pages 405-417. ACM, 2017.

[138] Benjamin D Horne and Sibel Adali. This just in: fake news packs a lot in title, uses simpler, repetitive content in text body, more similar to satire than real news. arXiv preprint arXiv:1703.09398, 2017.

[139] Victoria Rubin, Niall Conroy, Yimin Chen, and Sarah Cornwell. Fake news or truth? using satirical cues to detect potentially misleading news. In Proceedings of the Second Workshop on Computational Approaches to Deception Detection, pages 7-17, 2016.

[140] Jonathan Albright. Data is the real post-truth, so hereâĂŹs the truth about post-\# election 2016 propaganda, 2016.

[141] Jonathan Albright. The\# election2016 micro-propaganda machine. Medium, 2016.

[142] Kai Shu, Suhang Wang, and Huan Liu. Exploiting tri-relationship for fake news detection. arXiv preprint arXiv:1712.07709, 2017. 
[143] William Yang Wang. " liar, liar pants on fire": A new benchmark dataset for fake news detection. arXiv preprint arXiv:1705.00648, 2017.

[144] Clayton Allen Davis, Onur Varol, Emilio Ferrara, Alessandro Flammini, and Filippo Menczer. Botornot: A system to evaluate social bots. In Proceedings of the 25th International Conference Companion on World Wide Web, pages 273-274. International World Wide Web Conferences Steering Committee, 2016.

[145] Alex Krizhevsky, Ilya Sutskever, and Geoffrey E Hinton. Imagenet classification with deep convolutional neural networks. Advances in neural information processing systems, 25:1097-1105, 2012.

[146] Dario Amodei, Sundaram Ananthanarayanan, Rishita Anubhai, Jingliang Bai, Eric Battenberg, Carl Case, Jared Casper, Bryan Catanzaro, Qiang Cheng, Guoliang Chen, et al. Deep speech 2: End-to-end speech recognition in english and mandarin. In International conference on machine learning, pages 173-182. PMLR, 2016.

[147] Jun Wang, Lantao Yu, Weinan Zhang, Yu Gong, Yinghui Xu, Benyou Wang, Peng Zhang, and Dell Zhang. Irgan: A minimax game for unifying generative and discriminative information retrieval models. In Proceedings of the 40th International ACM SIGIR conference on Research and Development in Information Retrieval, pages 515-524, 2017.

[148] Thomas N Kipf and Max Welling. Semi-supervised classification with graph convolutional networks. arXiv preprint arXiv:1609.02907, 2016.

[149] Xiang Zhang, Junbo Zhao, and Yann LeCun. Character-level convolutional networks for text classification. Advances in neural information processing systems, 28:649-657, 2015.

[150] Qiang Zhang, Shangsong Liang, and Emine Yilmaz. Variational self-attention model for sentence representation. arXiv preprint arXiv:1812.11559, 2018. 
[151] Dirk Walther, Ueli Rutishauser, Christof Koch, and Pietro Perona. On the usefulness of attention for object recognition. In Workshop on Attention and Performance in Computational Vision at ECCV, pages 96-103, 2004.

[152] Xiaolong Wang, Ross Girshick, Abhinav Gupta, and Kaiming He. Non-local neural networks. In Proceedings of the IEEE Conference on Computer Vision and Pattern Recognition, pages 7794-7803, 2018.

[153] Diederik P. Kingma and Max Welling. Auto-encoding variational bayes. CoRR, abs/1312.6114, 2013.

[154] David Roxbee Cox and Valerie Isham. Point processes, volume 12. CRC Press, 1980.

[155] Daryl J Daley and David Vere-Jones. An introduction to the theory of point processes: volume II: general theory and structure. Springer Science \& Business Media, 2007.

[156] Jakob Gulddahl Rasmussen. Lecture notes: Temporal point processes and the conditional intensity function. arXiv preprint arXiv:1806.00221, 2018.

[157] Sung Nok Chiu, Dietrich Stoyan, Wilfrid S Kendall, and Joseph Mecke. Stochastic geometry and its applications. John Wiley \& Sons, 2013.

[158] John Hawkes. On the hausdorff dimension of the intersection of the range of a stable process with a borel set. Probability Theory and Related Fields, 19(2):90-102, 1971.

[159] Mehrdad Farajtabar, Nan Du, Manuel Gomez Rodriguez, Isabel Valera, Hongyuan Zha, and Le Song. Shaping social activity by incentivizing users. In Advances in neural information processing systems, pages 2474-2482, 2014.

[160] Ke Zhou, Hongyuan Zha, and Le Song. Learning social infectivity in sparse low-rank networks using multi-dimensional hawkes processes. In Artificial Intelligence and Statistics, pages 641-649, 2013. 
[161] Hongteng Xu, Mehrdad Farajtabar, and Hongyuan Zha. Learning granger causality for hawkes processes. In International Conference on Machine Learning, pages 1717-1726, 2016.

[162] Valerie Isham and Mark Westcott. A self-correcting point process. Stochastic Processes and Their Applications, 8(3):335-347, 1979.

[163] Shuai Xiao, Mehrdad Farajtabar, Xiaojing Ye, Junchi Yan, Le Song, and Hongyuan Zha. Wasserstein learning of deep generative point process models. In Advances in Neural Information Processing Systems, pages 32473257, 2017.

[164] Shuang Li, Shuai Xiao, Shixiang Zhu, Nan Du, Yao Xie, and Le Song. Learning temporal point processes via reinforcement learning. In Advances in Neural Information Processing Systems, pages 10781-10791, 2018.

[165] Hongyuan Mei and Jason M Eisner. The neural hawkes process: A neurally self-modulating multivariate point process. In Advances in Neural Information Processing Systems, pages 6754-6764, 2017.

[166] Qiang Zhang, Shangsong Liang, Aldo Lipani, Zhaochun Ren, and Emine Yilmaz. From stances' imbalance to their hierarchical representation and detection. In Companion Proceedings of the The Web Conference 2019. ACM Press, 2019.

[167] Peter Bourgonje, Julian Moreno Schneider, and Georg Rehm. From clickbait to fake news detection: an approach based on detecting the stance of headlines to articles. In Proceedings of the 2017 EMNLP Workshop: Natural Language Processing meets Journalism, pages 84-89, 2017.

[168] Nasser M Nasrabadi. Pattern recognition and machine learning. Journal of electronic imaging, 16(4):049901, 2007.

[169] Xuezhi Wang, Cong Yu, Simon Baumgartner, and Flip Korn. Relevant document discovery for fact-checking articles. In Companion of the The Web 
Conference 2018 on The Web Conference 2018, pages 525-533. International World Wide Web Conferences Steering Committee, 2018.

[170] Andreas Hanselowski, Avinesh PVS, Benjamin Schiller, Felix Caspelherr, Debanjan Chaudhuri, Christian M. Meyer, and Iryna Gurevych. A retrospective analysis of the fake news challenge stance-detection task. In Proceedings of the 27th International Conference on Computational Linguistics, pages 1859-1874. Association for Computational Linguistics, 2018.

[171] Sean Baird, Sibley Doug, and Yuxi Pan. Talos targets disinformation with fake news challenge victory. 2017.

[172] Andreas Hanselowski, PVS Avinesh, Benjamin Schiller, and Felix Caspelherr. Description of the system developed by team athene in the FNC-1. Technical report, Technical report, 2017.

[173] Samir Bajaj. "the pope has a new baby!" fake news detection using deep learning. 2017.

[174] Neel Rakholia and Shruti Bhargava. "is it true?" - deep learning for stance detection in news. 2017.

[175] Solomon Kullback and Richard A Leibler. On information and sufficiency. The annals of mathematical statistics, 22(1):79-86, 1951.

[176] Karsten M Borgwardt, Arthur Gretton, Malte J Rasch, Hans-Peter Kriegel, Bernhard Schölkopf, and Alex J Smola. Integrating structured biological data by kernel maximum mean discrepancy. Bioinformatics, 22(14):e49e57, 2006.

[177] Bo Geng, Dacheng Tao, and Chao Xu. Daml: Domain adaptation metric learning. IEEE Transactions on Image Processing, 20(10):2980-2989, 2011.

[178] Bernhard Schölkopf, Koji Tsuda, and Jean-Philippe Vert. Kernel methods in computational biology. MIT press, 2004. 
[179] Nachman Aronszajn. Theory of reproducing kernels. Transactions of the American mathematical society, 68(3):337-404, 1950.

[180] Arnold Neumaier. Solving ill-conditioned and singular linear systems: A tutorial on regularization. SIAM review, 40(3):636-666, 1998.

[181] Diederik P Kingma and Jimmy Ba. Adam: A method for stochastic optimization. arXiv preprint arXiv:1412.6980, 2014.

[182] Isabelle Augenstein, Tim Rocktäschel, Andreas Vlachos, and Kalina Bontcheva. Stance detection with bidirectional conditional encoding. In Proceedings of the 2016 Conference on Empirical Methods in Natural Language Processing, pages 876-885. Association for Computational Linguistics, 2016.

[183] Mitra Mohtarami, Ramy Baly, James Glass, Preslav Nakov, Lluís Màrquez, and Alessandro Moschitti. Automatic stance detection using end-to-end memory networks. arXiv preprint arXiv:1804.07581, 2018.

[184] Laurens van der Maaten and Geoffrey Hinton. Visualizing data using t-sne. Journal of machine learning research, 9(Nov):2579-2605, 2008.

[185] Jerry R Hobbs. Granularity. In Readings in qualitative reasoning about physical systems, pages 542-545. Elsevier, 1990.

[186] Stephen C Johnson. Hierarchical clustering schemes. Psychometrika, 32(3):241-254, 1967.

[187] Ling Zhang and Bo Zhang. Hierarchical machine learning-a learning methodology inspired by human intelligence. In International Conference on Rough Sets and Knowledge Technology, pages 28-30. Springer, 2006.

[188] Frederic Morin and Yoshua Bengio. Hierarchical probabilistic neural network language model. In International workshop on artificial intelligence and statistics, pages 246-252. PMLR, 2005. 
[189] Nathalie Japkowicz and Shaju Stephen. The class imbalance problem: A systematic study. Intelligent data analysis, 6(5):429-449, 2002.

[190] Qiang Zhang, Aldo Lipani, Shangsong Liang, and Emine Yilmaz. Replyaided detection of misinformation via bayesian deep learning. In Companion Proceedings of the The Web Conference 2019. ACM Press, 2019.

[191] Yishu Miao, Lei Yu, and Phil Blunsom. Neural variational inference for text processing. In International conference on machine learning, pages 17271736. PMLR, 2016.

[192] Pedro Domingos. Bayesian averaging of classifiers and the overfitting problem. In ICML, volume 747, pages 223-230. Citeseer, 2000.

[193] William Yang Wang. "liar, liar pants on fire": A new benchmark dataset for fake news detection. In Proceedings of the 55th Annual Meeting of the Association for Computational Linguistics (Volume 2: Short Papers), pages 422-426. Association for Computational Linguistics, 2017.

[194] Alex Graves, Santiago Fernández, and Jürgen Schmidhuber. Bidirectional $1 s t m$ networks for improved phoneme classification and recognition. In International Conference on Artificial Neural Networks, pages 799-804. Springer, 2005.

[195] Chong Wang and David M. Blei. Variational inference in nonconjugate models. J. Mach. Learn. Res., 14(1):1005-1031, April 2013.

[196] Danilo Jimenez Rezende, Shakir Mohamed, and Daan Wierstra. Stochastic backpropagation and approximate inference in deep generative models. In Eric P. Xing and Tony Jebara, editors, Proceedings of the 31st International Conference on Machine Learning, volume 32 of Proceedings of Machine Learning Research, pages 1278-1286, Bejing, China, 22-24 Jun 2014. PMLR. 
[197] Frank Hansen and Gert K Pedersen. Jensen's operator inequality. Bulletin of the London Mathematical Society, 35(4):553-564, 2003.

[198] Leon Derczynski, Kalina Bontcheva, Maria Liakata, Rob Procter, Geraldine Wong Sak Hoi, and Arkaitz Zubiaga. Semeval-2017 task 8: Rumoureval: Determining rumour veracity and support for rumours. In Proceedings of the 11th International Workshop on Semantic Evaluation (SemEval-2017), pages 69-76. Association for Computational Linguistics, 2017.

[199] Nitish Srivastava. Improving neural networks with dropout. University of Toronto, 182:566, 2013.

[200] Cicero dos Santos and Maira Gatti. Deep convolutional neural networks for sentiment analysis of short texts. In Proceedings of COLING 2014, the 25th International Conference on Computational Linguistics: Technical Papers, pages 69-78, 2014.

[201] Yoon Kim. Convolutional neural networks for sentence classification. In Proceedings of the 2014 Conference on Empirical Methods in Natural Language Processing, EMNLP 2014, October 25-29, 2014, Doha, Qatar, A meeting of SIGDAT, a Special Interest Group of the ACL, pages 1746-1751, 2014.

[202] Yann N. Dauphin, Angela Fan, Michael Auli, and David Grangier. Language modeling with gated convolutional networks. In Proceedings of the 34th International Conference on Machine Learning, ICML 2017, Sydney, NSW, Australia, 6-11 August 2017, pages 933-941, 2017.

[203] Yi-Chin Chen, Zhao-Yang Liu, and Hung-Yu Kao. Ikm at semeval-2017 task 8: Convolutional neural networks for stance detection and rumor verification. In Proceedings of the 11th International Workshop on Semantic Evaluation, pages 465-469, 2017.

[204] Tomas Mikolov, Ilya Sutskever, Kai Chen, Greg S Corrado, and Jeff Dean. Distributed representations of words and phrases and their compositionality. 
In Advances in neural information processing systems, pages 3111-3119, 2013.

[205] Gisel Bastidas Guacho, Sara Abdali, Neil Shah, and Evangelos E Papalexakis. Semi-supervised content-based detection of misinformation via tensor embeddings. arXiv preprint arXiv:1804.09088, 2018.

[206] Kashyap Popat, Subhabrata Mukherjee, Andrew Yates, and Gerhard Weikum. Declare: Debunking fake news and false claims using evidenceaware deep learning. In Proceedings of the 2018 Conference on Empirical Methods in Natural Language Processing, Brussels, Belgium, October 31 November 4, 2018, pages 22-32, 2018.

[207] Jing Ma, Wei Gao, and Kam-Fai Wong. Rumor detection on twitter with tree-structured recursive neural networks. In Proceedings of the 56th Annual Meeting of the Association for Computational Linguistics (Volume 1: Long Papers), pages 1980-1989. Association for Computational Linguistics, 2018.

[208] Yarin Gal and Zoubin Ghahramani. Dropout as a bayesian approximation: Representing model uncertainty in deep learning. In international conference on machine learning, pages 1050-1059. PMLR, 2016.

[209] Balaji Lakshminarayanan, Alexander Pritzel, and Charles Blundell. Simple and scalable predictive uncertainty estimation using deep ensembles. arXiv preprint arXiv:1612.01474, 2016.

[210] Andrey Malinin and Mark Gales. Predictive uncertainty estimation via prior networks. arXiv preprint arXiv:1802.10501, 2018.

[211] Elena Kochkina and Maria Liakata. Estimating predictive uncertainty for rumour verification models. arXiv preprint arXiv:2005.07174, 2020.

[212] Qiang Zhang, Aldo Lipani, Omer Kirnap, and Emine Yilmaz. Self-attentive hawkes process. ICML, 2020. 
[213] Mehrdad Farajtabar, Yichen Wang, Manuel Gomez Rodriguez, Shuang Li, Hongyuan Zha, and Le Song. Coevolve: A joint point process model for information diffusion and network co-evolution. In Advances in Neural Information Processing Systems, pages 1954-1962, 2015.

[214] Emmanuel Bacry and Jean-François Muzy. Hawkes model for price and trades high-frequency dynamics. Quantitative Finance, 14(7):1147-1166, 2014.

[215] Yichen Wang, Bo Xie, Nan Du, and Le Song. Isotonic hawkes processes. In International conference on machine learning, pages 2226-2234, 2016.

[216] Patricia Reynaud-Bouret, Sophie Schbath, et al. Adaptive estimation for hawkes processes; application to genome analysis. The Annals of Statistics, 38(5):2781-2822, 2010.

[217] Yosihiko Ogata. Space-time point-process models for earthquake occurrences. Annals of the Institute of Statistical Mathematics, 50(2):379-402, 1998.

[218] David R Brillinger, Peter M Guttorp, Frederic Paik Schoenberg, Abdel H ElShaarawi, and Walter W Piegorsch. Point processes, temporal. Encyclopedia of Environmetrics, 3:1577-1581, 2002.

[219] Xinran He, Theodoros Rekatsinas, James Foulds, Lise Getoor, and Yan Liu. Hawkestopic: A joint model for network inference and topic modeling from text-based cascades. In International conference on machine learning, pages 871-880, 2015.

[220] Nan Du, Mehrdad Farajtabar, Amr Ahmed, Alexander J Smola, and Le Song. Dirichlet-hawkes processes with applications to clustering continuous-time document streams. In Proceedings of the 21th ACM SIGKDD International Conference on Knowledge Discovery and Data Mining, pages 219228. ACM, 2015. 
[221] Shuang-Hong Yang and Hongyuan Zha. Mixture of mutually exciting processes for viral diffusion. In International Conference on Machine Learning, pages 1-9, 2013.

[222] Edward Choi, Nan Du, Robert Chen, Le Song, and Jimeng Sun. Constructing disease network and temporal progression model via context-sensitive hawkes process. In 2015 IEEE International Conference on Data Mining, pages 721-726. IEEE, 2015.

[223] Jalal Etesami, Negar Kiyavash, Kun Zhang, and Kushagra Singhal. Learning network of multivariate hawkes processes: A time series approach. In Proceedings of the Thirty-Second Conference on Uncertainty in Artificial Intelligence, UAI'16. AUAI Press, 2016.

[224] Nan Du, Yichen Wang, Niao He, Jimeng Sun, and Le Song. Time-sensitive recommendation from recurrent user activities. In Advances in Neural Information Processing Systems, pages 3492-3500, 2015.

[225] Fangjian Guo, Charles Blundell, Hanna Wallach, and Katherine Heller. The bayesian echo chamber: Modeling social influence via linguistic accommodation. In Artificial Intelligence and Statistics, pages 315-323, 2015.

[226] Nan Du, Hanjun Dai, Rakshit Trivedi, Utkarsh Upadhyay, Manuel GomezRodriguez, and Le Song. Recurrent marked temporal point processes: Embedding event history to vector. In Proceedings of the 22nd ACM SIGKDD International Conference on Knowledge Discovery and Data Mining, pages 1555-1564. ACM, 2016.

[227] Shuai Xiao, Junchi Yan, Xiaokang Yang, Hongyuan Zha, and Stephen M Chu. Modeling the intensity function of point process via recurrent neural networks. In Thirty-First AAAI Conference on Artificial Intelligence, 2017.

[228] Takahiro Omi, Naonori Ueda, and Kazuyuki Aihara. Fully neural network based model for general temporal point processes. arXiv preprint arXiv:1905.09690, 2019. 
[229] Oleksandr Shchur, Marin Biloš, and Stephan Günnemann. Intensity-free learning of temporal point processes. arXiv preprint arXiv:1909.12127, 2019.

[230] Andrej Karpathy, Justin Johnson, and Li Fei-Fei. Visualizing and understanding recurrent networks. arXiv preprint arXiv:1506.02078, 2015.

[231] Viktoriya Krakovna and Finale Doshi-Velez. Increasing the interpretability of recurrent neural networks using hidden markov models. arXiv preprint arXiv:1611.05934, 2016.

[232] Peter Shaw, Jakob Uszkoreit, and Ashish Vaswani. Self-attention with relative position representations. arXiv preprint arXiv:1803.02155, 2018.

[233] Xing Wang, Zhaopeng Tu, Longyue Wang, and Shuming Shi. Self-attention with structural position representations. arXiv preprint arXiv:1909.00383, 2019.

[234] Dan Hendrycks and Kevin Gimpel. Gaussian error linear units (gelus). arXiv preprint arXiv:1606.08415, 2016.

[235] Yosihiko Ogata. On lewis' simulation method for point processes. IEEE Transactions on Information Theory, 27(1):23-31, 1981.

[236] Qiang Zhang, Jonathan Cook, and Emine Yilmaz. Detecting and forecasting misinformation via temporal and geometric propagation patterns. In ECIR (2), pages 455-462, 2021.

[237] Federico Monti, Fabrizio Frasca, Davide Eynard, Damon Mannion, and Michael M Bronstein. Fake news detection on social media using geometric deep learning. arXiv preprint arXiv:1902.06673, 2019.

[238] Yi-Ju Lu and Cheng-Te Li. Gcan: Graph-aware co-attention networks for explainable fake news detection on social media. arXiv preprint arXiv:2004.11648, 2020. 
[239] Yuanfu Lu, Xiao Wang, Chuan Shi, Philip S. Yu, and Yanfang Ye. Temporal network embedding with micro- and macro-dynamics. arXiv preprint arXiv:1909.04246, 2019.

[240] Sejeong Kwon, Meeyoung Cha, and Kyomin Jung. Rumor detection over varying time windows. PloS one, 12(1):e0168344, 2017.

[241] Yang Liu and Yi-Fang Brook Wu. Early detection of fake news on social media through propagation path classification with recurrent and convolutional networks. In Thirty-Second AAAI Conference on Artificial Intelligence, 2018.

[242] Kai Shu, Limeng Cui, Suhang Wang, Dongwon Lee, and Huan Liu. defend: Explainable fake news detection. In Proceedings of the 25th ACM SIGKDD International Conference on Knowledge Discovery \& Data Mining, pages 395-405, 2019. 